\title{
Brane world perspective on the cosmological constant and the hierarchy problems
}

\author{
Eanna Flanagan, ${ }^{*}$ Nicholas Jones ${ }^{\dagger}$ Horace Stoica, ${ }^{*}$ S.-H. Henry Tye, ${ }^{\S}$ and Ira Wasserman \\ Laboratory for Nuclear Studies and Center for Radiophysics and Space Research, Cornell University, Ithaca, New York 14853
}

(Received 11 January 2001; published 20 July 2001)

\begin{abstract}
We elaborate on the recently proposed static brane world scenario, where the effective 4D cosmological constant is exponentially small when parallel 3-branes are far apart. We extend this result to a compactified model with two positive tension branes. In addition to an exponentially small effective 4D cosmological constant, this model incorporates a Randall-Sundrum-like solution to the hierarchy problem. Furthermore, the exponential factors for the hierarchy problem and the cosmological constant problem obey an inequality that is satisfied in nature. This inequality implies that the cosmological constant problem can be explained if the hierarchy problem is understood. The basic idea generalizes to the multibrane world scenario. We discuss models with piecewise adjustable bulk cosmological constants (to be determined by the 5-dimensional Einstein equation), a key element of the scenario. We also discuss the global structure of this scenario and clarify the physical properties of the particle (Rindler) horizons that are present. Finally, we derive a 4D effective theory in which all observers on all branes not separated by particle horizons measure the same Newton's constant and 4D cosmological constant.
\end{abstract}

DOI: 10.1103/PhysRevD.64.045007

PACS number(s): 11.10.Kk, 04.50. $+\mathrm{h}, 98.80 . \mathrm{Hw}$

\section{INTRODUCTION}

Recent observational data [1] indicate that our Universe has a positive cosmological constant which, compared to the Planck or the electroweak scale, is many orders of magnitude smaller than expected within the context of ordinary gravity and quantum field theory. This is the well-known cosmological constant problem [2,3]. Recently, two of us proposed a static brane world solution to this problem in which the cosmological constant becomes exponentially small compared to all other scales in the model [4]. Here, by "static" we mean the situation where the branes are stationary relative to each other. ${ }^{1}$ We still allow for expansion of the Universe in the other three spatial directions, but confine ourselves to situations in which the Hubble expansion rate $H$ is time independent. In more complete big bang cosmological models, this would correspond to late times, when the cosmological constant becomes dominant.

It is generally believed that fine-tuning is necessary for a very small cosmological constant in 4-dimensional theories [2]. This leads one to search for a naturally small cosmological constant in higher dimensional theories. However, for a usual compactification of a higher dimensional theory to an effective 4-dimensional theory, one ends up with a normal 4-dimensional theory, and the fine-tuning problem generically reappears. This is the case for usual Kaluza-Klein (KK)

\footnotetext{
*Electronic address: flanagan@spacenet.tn.cornell.edu

${ }^{\dagger}$ Electronic address: nicholas-jones@ cornell.edu

ॠElectronic address: fhs3@mail.lns.cornell.edu

${ }^{\S}$ Electronic address: tye@mail.lns.cornell.edu

"Electronic address: ira@spacenet.tn.cornell.edu

${ }^{1}$ More precisely, the bulk spacetime possesses a timelike Killing vector field which is not hypersurface orthogonal, so that the spacetime is stationary but not static, and the branes are invariant under the diffeomorphism generated by this vector field.
}

compactification, and for the generic compactifications with large extra dimensions [5]. ${ }^{2}$ The Randall-Sundrum (RS) model $[7,8]$ provides a hope of avoiding this pathology. The RS model considers 3-branes inside 5-dimensional spacetime, with anti-de Sitter (AdS) spaces in the bulk. Normal finite gravitational interaction is reproduced even if the 5 th spatial dimension is not compactified. For a single 3-brane (or a stack of 3-branes) with brane tension (or vacuum energy density) $\sigma_{0}$, the effective 4D cosmological constant as seen by observers on the brane is taken to be zero, that is $[7,9]$

$$
\Lambda_{e f f} \propto \sigma_{0}-\sqrt{6 \Lambda / \kappa^{2}}=0
$$

where $\kappa^{2}$ is the 5-dimensional gravitational coupling, and $-\Lambda$ is the negative bulk cosmological constant. ${ }^{3}$ We see that the brane tension, which is at least partly determined by the dynamics of the standard model, is not required to vanish. The cancellation between the brane tension and the bulk cosmological constant is imposed in any brane world scenario. However, the non-linear relation (i.e., $\sigma_{0}$ versus $\sqrt{\Lambda}$ ), as well as the lack of the need to compactify the 5 th dimension, follow from the non-factorizable metric in the RS model. Since the 5 th dimension may stay uncompactified, any 4D description will be inadequate. If one now insists on a 4D description for observers on the brane, one ends up with a novel 4D description via the AdS conformal field theory (CFT) correspondence $[10,11]$, which offers hope to void the argument for the need of fine-tuning to obtain a very small 4D cosmological constant. This has motivated a number of attempts to find a solution to the cosmological constant prob-

\footnotetext{
${ }^{2}$ See however Ref. [6] for a suggestion in the context of $4+2$ dimensions.

${ }^{3}$ Throughout this paper, the cosmological constants will have units of energy density in the appropriate dimension.
} 
lem along this direction [12]. Here we shall elaborate on the approach of Ref [4]. In the two brane Randall-Sundrum model [7], the cancellation in Eq. (1) is a fine-tuning. In addition, the two branes are required to have equal and opposite tensions. These two fine-tunings are removed in Ref. [4].

The two brane model in Ref. [4] does not solve the hierarchy problem, which is presumably solved in the RS model. However, the two brane model of Ref. [4] is easy to generalize to a multibrane model in which both the cosmological constant problem and the hierarchy problem can be explained simultaneously. The generalization to $N 3$-branes is straightforward because an observer on any brane can only see properties around his or her own neighborhood. This allows us to analyze the various possibilities generally.

The simplest realization that solves both fine-tuning problems is a two brane compactified model. The model has an exponentially small $\Lambda_{\text {eff }}$, as in Ref. [4], and implements the Randall-Sundrum-like solution to the hierarchy problem, but with a positive tension for the visible brane. Consider two parallel 3-branes with tensions $\sigma_{0}>\sigma_{1}>0$ sitting in a compactified 5th dimension, with radius $L_{2} / 2 \pi$. The $\sigma_{0}$ (Planck, hidden) brane sits at $L_{0}=0$ and the $\sigma_{1}(\mathrm{TeV}$, visible) brane sits at $L_{1}$. Since $L_{2}$ is identified with $L_{0}$, this means the branes are separated by $L_{1}$ on one side and by $L_{2}-L_{1}$ on the other side. Without loss of generality, let $L_{2}-L_{1}>L_{1}$. We find that, for large $L_{1}$, the warp factor for the hierarchy problem is

$$
\frac{m_{\text {Higgs }}^{2}}{m_{\text {Planck }}^{2}} \simeq \frac{A\left(L_{1}\right)}{A(0)} \simeq e^{-\kappa^{2}\left(\sigma_{0}-\sigma_{1}\right) L_{1} / 3}
$$

and the effective $4 \mathrm{D}$ cosmological constant is

$$
\Lambda_{e f f} \simeq \frac{2 \sigma_{0}\left(\sigma_{0}+\sigma_{1}\right)}{\left(\sigma_{0}-\sigma_{1}\right)} e^{-\kappa^{2}\left[\left(\sigma_{0}+\sigma_{1}\right)\left(L_{2}-L_{1}\right)+\left(\sigma_{0}-\sigma_{1}\right) L_{1}\right] / 6} .
$$

Since $\sigma_{0}>\sigma_{1}>0$, and $L_{2}-L_{1}>L_{1}$, we see that

$$
\ln \left(\frac{m_{\text {Planck }}^{4}}{\Lambda_{\text {eff }}}\right)>\ln \left(\frac{m_{\text {Planck }}^{2}}{m_{\text {Higgs }}^{2}}\right)
$$

as is the case in nature, where $\ln \left(m_{\text {Planck }}^{4} / \Lambda_{\text {eff }}\right) \sim 2.3 \times 122$ and $\ln \left(m_{\text {Planck }}^{2} / m_{\text {Higgs }}^{2}\right) \sim 2.3 \times 32$. To get a feeling for the magnitudes of the various quantities, we may choose $L_{2}-L_{1}$ $\sim 2 L_{1}$, and $\sigma_{0} \sim 2 \sigma_{1}$. If the brane tensions are around the Planck scale, the exponents that describe nature follow if $L_{1} \sim 10 m_{\text {Planck. }}^{-1}$. In this model, the branes are stationary (that is, they satisfy the brane equations of motion) and both $L_{1}$ and $L_{2}$ are stable. But we still need a dynamical reason why $L_{1}$ (or $L_{2}$ ) is large. However, if we understand the hierarchy problem via some means [e.g., comparing the renormalization group flows of couplings (marginal operators) versus the mass terms (relevant operators) in 4D quantum field theory], this brane world model provides an explanation of the cos- mological constant problem. As we shall see, the above inequality (4) is robust in a generic multibrane world where the hierarchy problem is solved.

The exponential behaviors are related to the warp factor in the Randall-Sundrum scenario, but with two key differences:

(1) The RS model demands $\Lambda_{\text {eff }}$ to be exactly zero. This requires a fine-tuning, namely, $\sigma_{0}+\sigma_{1}=0$. Here we find that this fine-tuning is not necessary for an exponentially small $\Lambda_{\text {eff }}$, as long as the branes are relatively far apart.

(2) The RS model also requires the fine-tuning in Eq. (1). Not surprisingly, although $\kappa^{2} \sigma_{0}^{2}-6 \Lambda$ in the above model [4] is not zero, it turns out to be exponentially small. A priori, this is still a fine-tuning. To avoid this fine-tuning, the bulk $\Lambda$ is treated as an "integration constant" that is determined by the 5D Einstein equation. There are a number of well-known realizations where the cosmological constant is an integration constant [13-21]. Since they are crucial to the above model, we shall review some of them, and then generalize them so that the bulk cosmological constant is piecewise constant, and each piece is an independent integration constant to be determined by the 5D Einstein equation. We note that this idea can just as easily be incorporated into the RS model.

The idea of treating the cosmological constant as an "integration constant" has a long history [2]. Classically, it is not determined and so the cosmological constant problem is not solved. In the brane world, it is the bulk cosmological constant that is treated as an "integration constant." In the single brane model, this bulk cosmological constant and so the effective 4D cosmological constant are undetermined, so it takes a fine-tuning to suppress the 4D cosmological constant. When the metric is non-factorizable, the introduction of another brane provides additional constraints which fix the value(s) of the bulk cosmological constant and hence the effective 4D cosmological constant as seen by observers on the brane. In this sense, the multibrane world scenario is key to the solution. In Ref. [4], the bulk cosmological constant is treated as an integration constant in a generic modelindependent way. Here, more details are worked out for the introduction of 5-form field strengths and its generalization. In specific models, we have additional equations, namely, an equation of motion for each brane. A priori, these equations are non-trivial for branes that are charged under the 4-form potential. We show that the static solution in Ref. [4] automatically satisfies these brane equations of motion.

If the number of parallel 3-branes is not too large, sitting in the uncompactified (or compactified with large radius) 5 th dimension, then we have a low density of 3-branes and their mean separation will be large in general. In the compactified version, observers on each brane will see an exponentially small effective 4D cosmological constant. In the uncompactified case, all but one brane will have this property. In this sense, the smallness of $\Lambda_{e f f}$ is quite generic.

In many cases, the metric between branes vanishes somewhere. These metric zeros are particle horizons which we show are coordinate singularities analogous to those in Rindler space. We eliminate those singularities, and deduce the global structure of the maximal extensions of the bulk space- 
times. We show that bulk regions separated by particle horizons often lie in separate connected components of the maximally extended solution, and therefore cannot physically influence one another.

In the multibrane model, an issue arises concerning the proper method for determining the 4D Newton's constant $G_{N}$. The two standard methods to determine $G_{N}$ are either to solve for the trapped gravity mode and Green's function, or to calculate the Hubble constant and determine the coefficient of the matter density term [9]. Naively, one would expect the normalization of the trapped gravity mode, and hence its wave function at a particular brane, to depend on the total number and placement of all of the branes, and so should the value of $G_{N}$ found from the trapped mode. By contrast, the value deduced from the cosmological expansion rate should be determined locally, by the brane tension and the bulk cosmological constants on either side of the brane, and, one would think, should not depend on how many other branes there are, or where they are located. The resolution is that the normalization of the trapped gravity mode (or any other mode) should be computed only over the region between particle horizons. This also implies that the integration over the 5 th dimension in the 5D action to obtain the lowenergy effective 4D action [7] should be carried out over the same region. This means that we cannot compare the mass scales between branes that are separated by particle horizons, which are normalized independently. Any solution to the mass hierarchy problem can be addressed only between branes that are not separated by particle horizons.

Now we can see the generic origin of the inequality (4). To solve the hierarchy problem, both the Planck and the visible branes must be inside the same particle horizons. The cosmological constant is exponentially small roughly as a function of the distance of the Planck brane from the particle horizon while the hierarchy scale (warp) factor is exponentially small roughly as a function of the distance of the Planck brane from the visible brane. Since the visible brane must be between the Planck brane and the particle horizon, the inequality (4) follows.

Using the effective 4D action approach, we show that both $G_{N}$ and the effective cosmological constant $\Lambda_{\text {eff }}$ are the same for all observers on all branes within a pair of particle horizons, irrespective of the type of brane (positive or negative tension, Planck or visible) on which they live. Generically, the visible brane will have different bulk cosmological constants on its two sides. The correction to the Newton's law is calculated in this case; it has the same dependence on particle separation as in the symmetric case [8], but with a modified coefficient.

In general, $\Lambda_{e f f}\left(L_{i}\right)$ is expected to be more complicated than given above, because we expect additional nongravitational brane-brane interactions at small separations. For large separations, it is reasonable to assume that the inter-brane dynamics is dominated by pure gravity as described here. In a more realistic situation, the matter density on the visible brane (and dark matter on the other brane) should be included, and the branes should be allowed to move. We shall not consider these effects in this paper. However, we do note that if the kinds of explanations offered here are indeed behind the smallness of the cosmological constant and Higgs boson mass relative to the Planck scale, branes must have moved very little since the epoch of cosmological nucleosynthesis. Otherwise, the Higgs boson mass scale might have changed enormously, which would be inconsistent with the success of big bang cosmology in explaining the light element abundances.

The paper is organized as follows. In Sec. II, we review models in which the bulk cosmological constant emerges as an "integration constant" and discuss the generalization where it becomes piecewise constant and piecewise adjustable. In Sec. III, we present the multibrane solution of the model and review the 4D effective action. Section IV reviews and elaborates on the two brane model of Ref. [4]. Section V considers a two brane compactified model where the Randall-Sundrum solution to the hierarchy problem is incorporated together with an exponentially small effective $4 \mathrm{D}$ cosmological constant. Section VI gives a general analysis of the multibrane world. Section VII discusses the global structure of the metric. In Sec. VIII, we discuss the implications of the particle horizons on the determination of $G_{N}$ and the mass hierarchy issue. Section IX considers the correction to Newton's gravity law in the case where the branes are sitting between two different AdS spaces. Section X contains some overall discussions and Sec. XI gives a brief summary. Some of the details are relegated to the Appendixes. Appendix A solves the brane equation of motion for a stationary brane. Appendix B derives some details of the two brane compactified model. Appendix C gives the details of the coordinate transformation used in the analysis of the global structure. For the visible brane in Ref. [7] or the two brane compactified model, a naive determination of Newton's constant using the Hubble constant approach sometimes yields a wrong result. In Appendix D, we comment on the ambiguity or problem with this naive approach. Appendix E gives a Feynman diagram analysis of Newton's force law on the relation between different metrics.

In this paper, the important issue of brane stability and brane motion are not discussed. Although the exactly stationary branes treated here satisfy the brane equations of motion, the brane separations are unstable to small perturbations. This can already be seen by considering a probe brane (i.e. a brane with negligible brane tension), originally at $y=L$, but then perturbed slightly away from that position. For small displacements, one finds two modes, a stable one proportional to $e^{-4 H t}$, and an unstable mode proportional to $e^{H t}$. (These modes can also be found from linear stability analysis of the solutions presented here, as well as from expansions of fully nonlinear brane equations of motion; we shall present these results elsewhere.) For a probe brane, the unstable motion ultimately becomes nonlinear, and eventually the brane picks up speed, and approaches the limiting speed $\sqrt{A(y)}$ (corresponding to motion at the speed of light). As discouraging as this instability may sound, it is worthwhile to remember that it only acts on a timescale comparable to $H^{-1}$, which, in the solutions presented here for branes with tension only, is exponentially small, of order the (expansion) age of the Universe. Thus, in such models, it is possible that the instability is relatively unimportant until the distant future of 
the Universe. However, it remains to assess what happens in the more realistic situation, where, in addition to tension, branes possess ordinary material particles and fields that dominate the net cosmological constant at early times, as is required in standard cosmology. In that case, our preliminary results show that the stationary case is once again linearly unstable to a growing mode that scales with the expansion factor of the Universe, for simple models of the bulk, but that the nonlinear motion of branes can be complicated, and depends on specific assumptions about the matter content of the branes. We shall publish details of this analysis elsewhere.

Nevertheless, we believe that the solutions presented here remain significant for two essential reasons. First, they illustrate how the smallness of the cosmological constant and the mass hierarchy problem may be connected, in the sense that one simple scenario may solve both problems, and may even require simultaneous solution of both problems. Second, they are based on very simple models for the physics of the bulk space and for the compactification of the additional six dimensions of the full 11D spacetime. Given the desirable properties of our static solutions, it is worthwhile to search for more complicated theories of the bulk for which one recovers solutions like the ones presented here at late times, but without instabilities. We are currently engaged in exploratory calculations on this issue.

\section{BULK COSMOLOGICAL CONSTANTS AS INTEGRATION CONSTANTS}

One of the key points underlying the two brane model [4] is that the bulk cosmological constant is not an input parameter of the model, but an "integration constant" to be determined by the 5D Einstein equation for the bulk together with the boundary conditions at the branes.

Suppose the 5D theory discussed above arises from the compactification of a higher dimensional theory. Then the bulk cosmological constant, the brane tensions, as well as the brane charges depend on the various compactification radii. If we do not fix the compactification volume but instead make the compactification radii dynamical, this will provide a way to adjust, among other quantities, the bulk cosmological constant. This means that the bulk cosmological constant becomes dynamical. We will treat possible dynamical models for the brane world elsewhere.

Here we shall review two approaches in which this "integration constant" appears:

(i) unimodular gravity [13-15], which is suitable for the two brane model;

(ii) 5 -form field strength model [18-21], which is suitable for the multibrane model, either compactified or uncompactified, but not suitable for the orbifold version.

We then present a generalized model. Special cases of this generalized model reduce to the 5 -form field strength model and the fully covariantized variation of the unimodular gravity [17]. To adapt it for the multibrane orbifold model, we introduce brane couplings so that the bulk cosmological constant becomes piecewise constant. We note that 5 -form field strength also appears in gauged supergravity and/or superstring realizations of the RS scenario [22].
In $4 \mathrm{D}$ gravity, when the cosmological constant is an integration constant, it is typically left undetermined. In the multibrane model, the bulk 5D cosmological constant is piecewise constant, determined by the 5D Einstein equation, including the jump conditions at the branes and the brane equations of motion. As we shall see, the number of such adjustable bulk cosmological constants should be equal to the number of bulks between branes, so, for $N$ branes in either the uncompactified case or the $S^{1} / Z_{2}$ orbifold case, we require $N-1$ adjustable bulk cosmological constants. In the compactified version, we need $N$ adjustable bulk cosmological constants.

\section{A. Unimodular gravity}

It was first pointed out by Einstein [13] that the cosmological constant could arise as an integration constant if the original equations of general relativity were replaced by their trace-free forms. Einstein [13] had in mind traceless source terms due to electromagnetic fields alone, but the same traceless field equations are also obtained in "unimodular gravity" $[14,16]$, where the conformal part of the metric is constrained and is not allowed to vary. The fields in this theory consist of a metric $g_{a b}$, a fixed, background, non-dynamical volume form $\hat{\epsilon}_{a_{1} a_{2} \ldots a}$, and other matter fields. The action of the theory is the standard Einstein action that depends only on the metric and the matter fields. However, only metrics $g_{a b}$ whose volume forms coincide with the background volume form $\hat{\epsilon}$ are allowed. (An equivalent description of the restriction on the space of metrics is that only metrics whose determinants in a particular fixed coordinate system are -1 are allowed [14].) Unimodular gravity is consistent with a massless spin-2 graviton propagating with $[(d-1)(d-2)$ $-2] / 2$ polarizations in the linearized version of gravity in $d$-dimensions [14]. Varying the action over the allowed class of metrics yields in $d$ dimensions only the traceless part of Einstein's equation,

$$
R^{a b}-\frac{1}{d} g^{a b} R=\kappa^{2}\left(T^{a b}-\frac{1}{d} g^{a b} T\right)
$$

where $T=T_{a}^{a}$.

The standard argument of invariance of the action under arbitrary linearized coordinate transformations still applies and shows that energy-momentum tensor satisfies the usual conservation law,

$$
\nabla_{a} T^{a b}=0
$$

and of course the Bianchi identities,

$$
\nabla_{b}\left(R^{a b}-\frac{1}{2} g^{a b} R\right)=0
$$

still hold. Combining Eqs. (5)-(7) gives

$$
\nabla_{a}\left[(d / 2-1) R+\kappa^{2} T\right]=0 .
$$


Equation (8) implies that $(d / 2-1) R+\kappa^{2} T$ is a constant, which we will denote by $\kappa^{2} \Lambda d$. As was noted by Einstein [13], when the relation $(d / 2-1) R+\kappa^{2} T=\kappa^{2} \Lambda d$ is inserted in Eq. (5), the result is the full Einstein equation with cosmological constant $\Lambda$ :

$$
G^{a b}+\kappa^{2} \Lambda g^{a b}=\kappa^{2} T^{a b} .
$$

In this model, the cosmological constant $\Lambda$ is merely an integration constant, and has nothing to do with any input parameter in the action, the microphysics, or the (quantum) vacuum fluctuations. (Note that we may absorb any cosmological constant term inside $T^{a b}$ into $\Lambda$.) In a setup where $\Lambda$ is not determined, it becomes a free parameter. In standard 4D gravity, this altered status of the cosmological constant does not help to explain its smallness. However, in the two brane world scenario in $5 \mathrm{D}, \Lambda$ is no longer a free parameter. Rather, it is the bulk cosmological constant determined by the 5D Einstein equation and the boundary (jump) conditions at the branes [4].

The presence of a non-dynamical background field in unimodular gravity is of course an unattractive feature of the theory. However, it is possible to find other theories with no background fields which, classically and on-shell, are equivalent to unimodular gravity [15]. For example, one can treat $\Lambda$ as a dynamical field and introduce an appropriate Lagrangian multiplier (namely, a vector field) to render it spacetime-independent [17]. This theory is a special case of our generalized model of Sec. II C below.

\section{B. 5-form field strength}

Another way to get a piecewise constant bulk cosmological constant is to introduce $(d-1)$-form fields $[18-21]$ in $d$-dimensional spacetime. This scenario is natural in compactified versions in string or $\mathrm{M}$ theory, in which $D$-branes are charged. This permits multibrane scenarios with the cosmological constant taking different constant values in different regions of the spacetime. The discontinuities are due to charged $(d-2)$-branes, which act as sources for the $(d$ -1)-form field. A worldvolume action for these branes should, therefore, contain a Wess-Zumino term: the integral of the pullback of the $(d-1)$-form potential. In the multibrane world, the action $S$ consists of a bulk action containing gravity,with metric $g_{a b}$, coupled to a $(d-1)$-form potential $A_{(d-1)}$, with field strength $F_{(d)}=d A_{(d-1)}$, and a set of standard worldvolume actions of $(d-2)$-branes containing the above-mentioned WZ terms with dynamical coordinate fields $X_{n}^{a}$. This means the location of the $n$th brane in the bulk is given by the embedding functions $x^{a}=X_{n}^{a}\left(\xi_{n}^{\mu}\right)$. In addition, the $n$th brane possesses a brane metric $\gamma_{n}^{\mu \nu}$ which is a function of the brane coordinates $\xi_{n}^{\mu}$ (for $d=5, a=0,1,2,3,5$ and $\mu=0,1,2,3)$. The action for such a system is $[23,21]$

$$
\begin{aligned}
S= & \int d^{d} x \sqrt{|g|}\left[\frac{1}{2 \kappa^{2}} R-\Lambda-\frac{1}{2 \cdot d !} F_{(d)}^{2}\right] \\
& +\sum_{n}\left\{-\frac{1}{2} \sigma_{n} \int d^{(d-1)} \xi_{n} \sqrt{\left|\gamma_{n}\right|}\right. \\
& \times\left[\gamma_{n}^{\mu \nu} \partial_{\mu} X^{a} \partial_{\nu} X^{b} g_{a b}\left(X_{n}\right)-C\right]
\end{aligned}
$$

$$
\left.\begin{array}{l}
-\frac{e_{n}}{(d-1) !} \int d^{(d-1)} \xi_{n} A_{a_{1} \cdots a_{d-1}}\left(X_{n}\right) \\
\times \partial_{\mu_{1}} X^{a_{1}} \cdots \partial_{\mu_{d-1}} X^{a_{d-1}} \epsilon^{\mu_{1} \cdots \mu_{d-1}}
\end{array}\right\}
$$

where $\sigma_{n}$ and $e_{n}$ are the tension and the charge of the $n$th brane. The dimensionless constant $C$ must have the value $p$ -1 in order to recover the correct equations of motion (see below). The tensor density $\epsilon^{\mu_{1}} \ldots \mu_{d-1}$ is totally antisymmetric with $\epsilon^{01 \ldots(d-2)}=1$. Varying the action $S$ with respect to the metric, we have

$$
\begin{aligned}
G^{a b}= & \kappa^{2}\left\{-\Lambda g^{a b}+\frac{1}{(d-1) !}\right. \\
& \times\left(F^{a c_{1} \cdots c_{d-2}} F_{c_{1} \cdots c_{d-2}}^{b}-\frac{1}{2 d} g^{a b} F_{(d)}^{2}\right) \\
& -\sum_{n} \frac{\sigma_{n}}{\sqrt{|g|}} \int d^{(d-1)} \xi_{n} \sqrt{\left|\gamma_{n}\right|} \\
& \left.\times\left[\gamma_{n}^{\mu \nu} \partial_{\mu} X^{a} \partial_{\nu} X^{b} \delta^{(d)}\left(x-X_{n}\right)\right]\right\} .
\end{aligned}
$$

Varying the action $S$ with respect to $A_{(d-1)}$, the brane metric $\gamma_{\mu \nu}$ and the coordinates $X^{a}$, we have, respectively,

$$
\begin{gathered}
\sqrt{|g|} \nabla_{a} F^{a, b_{1} \cdots b_{d-1}}-\sum_{n} e_{n} \int d^{(d-1)} \xi_{n} \partial_{\mu_{1}} X^{b_{1}} \cdots \partial_{\mu_{d-1}} \\
\times X^{b_{d-1}} \epsilon^{\mu_{1} \cdots \mu_{d-1}} \delta^{(d)}\left(x-X_{n}\right)=0 \\
\gamma_{n \mu \nu}=\partial_{\mu} X_{n}^{a} \partial_{\nu} X_{n}^{b} g_{a b}\left(X_{n}\right)
\end{gathered}
$$

and the brane equation of motion for the $n$th brane

$$
\begin{aligned}
\sigma_{n} \sqrt{\left|\gamma_{n}\right|}\left[\nabla_{\mu} \nabla^{\mu} X_{n}^{a}+\Gamma_{b c}^{a} \partial_{\mu} X_{n}^{b} \partial_{\nu} X_{n}^{c} \gamma_{n}^{\mu \nu}\right] \\
\quad+\frac{e_{n}}{(d-1) !} F_{b_{1} \cdots b_{d-1}}^{a} \partial_{\mu_{1}} X_{n}^{b_{1}} \cdots \partial_{\mu_{d-1}} X_{n}^{b_{d-1}} \epsilon^{\mu_{1} \cdots \mu_{d-1}} \\
=0 .
\end{aligned}
$$

Equation (13) simply states that the world volume metrics are those induced on the world volumes by the embedding coordinates $X_{n}^{a}$. In Eq. (13) there is a coefficient $C /(p-1)$, for a $p$-brane ( $p=3$ in our case), which we have set equal to 1 , i.e., $\gamma_{\mu \nu}$ is the induced metric on the brane. Had we written the brane tension term in the Nambu-Goto action form, the same equations would have resulted. In the bulk

$$
\partial_{a}\left(\sqrt{|g|} F^{a c_{1}} \cdots c_{d-1}\right)=0
$$

which has solution 


$$
F^{a_{1} \cdots a_{d}}=\frac{e}{\sqrt{|g|}} \epsilon^{a_{1} \cdots a_{d}}
$$

where $e$ is a constant that can vary from one interbrane region to another, and the tensor density $\epsilon^{a_{1} \cdots a_{d}}$ is totally antisymmetric with $\epsilon^{01 \ldots(d-1)}=1$. Inserting this solution in Eq. (11) we see that the only contribution of the field strength is to the bulk cosmological constant $\Lambda$. In this paper we are interested in the parallel stationary 3-branes case, so we may choose the Gaussian normal coordinate: $d s^{2}$ $=g_{\mu \nu}(x, y) d x^{\mu} d x^{\nu}+d y^{2}$. The brane equation of motion (14) for a stationary brane is discussed in Appendix A. Using world volume reparametrization invariance we can set $d$ -1 coordinates (static gauge) to the values $X_{n}^{a}=\delta_{\mu}^{a} \xi_{n}^{\mu}$. The remaining coordinate, namely $X^{d} \equiv y$, is along the $d$ th direction,

$$
X_{n}^{d} \equiv L_{n},
$$

where the $L_{n}$ 's are constants. We can perform the volume integrals in Eqs. (11),(12) leaving only 1-dimensional delta functions $\delta\left(y-L_{n}\right)$. Also, this implies for the world volume metrics that $\gamma_{n \mu \nu}=g_{\mu \nu}\left(L_{n}\right)$. This can also be seen by solving Eq. (13) in the Gaussian normal system of coordinates in the bulk. Since the branes are parallel, the geodesics orthogonal to one brane will be orthogonal to all the branes. We obtain

$$
\gamma_{n \mu \nu}=\partial_{\mu} \xi_{n}^{a} \partial_{\nu} \xi_{n}^{b} g_{a b}\left(X_{n}\right)=g_{\mu \nu}\left(L_{n}\right)
$$

where we have taken $g_{5 \lambda}=0$ for Gaussian normal coordinates. It follows that $\sqrt{|\gamma|}=\sqrt{|g|}$, and Eq. (12) becomes

$$
\sqrt{|g|} \nabla_{a} F^{a, b_{1} \cdots b_{d-1}}-\sum_{n} e_{n} \epsilon^{b_{1} \cdots b_{d-1}} \delta\left(y-L_{n}\right)=0 .
$$

The determinant of the metric is a continuous function everywhere in the space, so as we go across the $n$th brane with charge $e_{n}$, the field strength jumps by $e_{n}$, from $e_{(n)}$ to $e_{(n+1)}=e_{(n)}+e_{n}$. In the multibrane world, the resulting cosmological constant in the bulk between the $(n-1)$ th and the $n$th branes is given by

$$
-\Lambda_{n}=\Lambda+\frac{1}{2} e_{(n)}^{2}=\Lambda+\frac{1}{2}\left(e_{(0)}+e_{0}+e_{1}+\cdots+e_{n-1}\right)^{2},
$$

where $e_{(0)}$ is a constant background field strength. Now the Einstein equation (11) becomes

$$
\begin{aligned}
G^{a b}= & \kappa^{2}\left[\sum_{n=0}^{N} \Lambda_{n} \Theta\left(y-L_{n-1}\right) \Theta\left(L_{n}-y\right) g^{a b}\right. \\
& \left.-\sum_{n=0}^{N-1} \frac{\sigma_{n}}{2} \delta_{\mu}^{a} \delta_{\nu}^{b} \gamma^{\mu \nu} \delta\left(y-L_{n}\right)\right]
\end{aligned}
$$

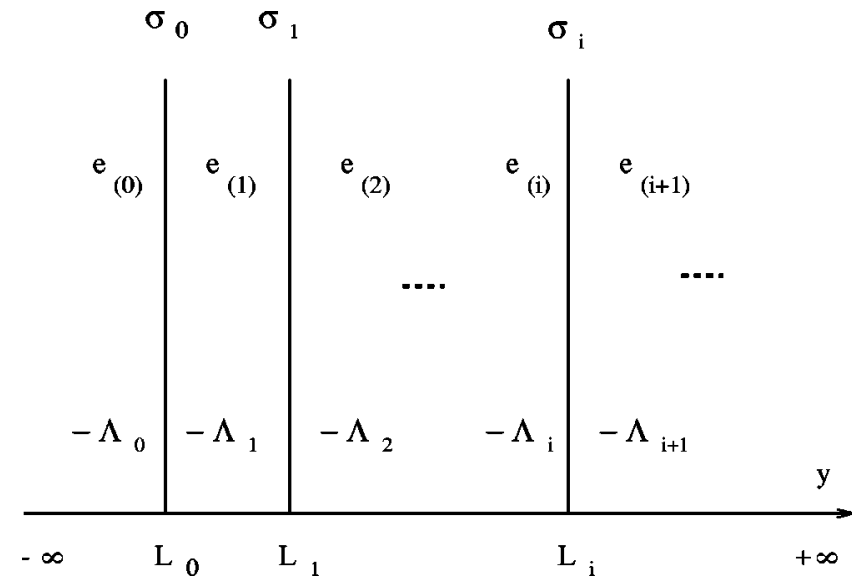

FIG. 1. Schematic multibrane setup.

where $\Lambda_{n}>0$ for AdS space, with $-L_{-1}=L_{N}=\infty$ in the uncompactified case. The multibrane world with an uncompactified 5 th dimension is shown in Fig. 1.

Although the charges $e_{n}$ of the branes are taken to be fixed, the background field strength $e_{(0)}$ is an integration constant (to be determined by the Einstein equation). To have AdS spaces in the bulk, we require $\Lambda$ to be negative enough so that all $\Lambda_{n}>0$. Since the $(d-1)$-form field has no dynamical degrees of freedom, it is consistent to flip the $F^{2}$ term in the action $S$ so that the field strength contributes only negatively to the bulk cosmological constant, avoiding the need to introduce a negative $\Lambda$. However, if the $(d-1)$-form field arises as a component of an antisymmetric field in higher dimensional space, then positivity of its higher dimensional kinetic energy density does not allow this freedom.

Suppose the $d$ th dimension (i.e., the $y$ direction) is compactified in the $N 3$-brane scenario. Boundary condition requires the sum of the brane charges to be zero, that is, $e_{0}$ $+e_{1}+e_{2}+\cdots+e_{N}=0$, independent of the value of $e_{(0)}$. If the model is a $S^{1} / Z_{2}$ orbifold, the symmetry constraint requires that $e_{(0)}=0$. In this case, the bulk $\Lambda_{n}$ are interrelated, so we have to find another way to allow $\Lambda_{n}$ to be adjustable.

In the two brane world, $e_{(0)}$ is determined by the Einstein equation. In the multibrane world, we may need more freedom than one integration constant. To achieve this, we can consider a scenario where there is more than one $(d-1)$-form field. Considering the case $d=5$, the introduction of more than one 4-form field is quite natural in string or $M$ theory. To be specific, consider $M$ theory which has 2-branes and 5-branes, which are electrically and magnetically charged, respectively, under the 3 -form field $A_{(3)}$. The dual of this 3 -form field is a 6 -form field $A_{(6)}$. If 6 of the 11 dimensions of $\mathrm{M}$ theory are compactified toroidally, then of the 6-form field $A_{(6)}$ there are, among other fields, 154 -form fields $A_{(4)}^{J}(J=1,2, \ldots, 15)$ in the uncompactified 5D spacetime. The 3-branes in this remaining $5 \mathrm{D}$ spacetime are 5 -branes of the 11D theory with two of their spatial dimensions compactified. There are 15 types of such 3-branes, each charged under one 4 -form field $A_{(4)}^{J}$. In more realistic compactifications, some of these 4-form fields may be projected out, although generically, we expect a number to remain. 
Let each 3-brane be a stack composed of these compactified 5-branes. Suppose we have $M 4$-form fields $A_{(4)}^{J}$ and their corresponding field strength $F_{(5)}^{J}, J=1,2, \ldots, M$, under which the $n$th 3 -brane has charge $e_{n}^{J}, J=1,2, \ldots, M$. Then the resulting cosmological constant in the bulk between the $(n-1)$ th and the $n$th branes is given by

$$
\begin{aligned}
-\Lambda_{n} & =\Lambda+\frac{1}{2} \sum_{J}\left(e_{(n)}^{J}\right)^{2} \\
& =\Lambda+\frac{1}{2} \sum_{J}\left(e_{(0)}^{J}+e_{0}^{J}+e_{1}^{J}+\cdots+e_{n-1}^{J}\right)^{2} .
\end{aligned}
$$

Again we require $\Lambda_{n}>0$ for AdS spaces between branes. Now there are $M$ background field strengths $e_{(0)}^{J}, J$ $=1,2, \ldots, M$, that are integration constants to be determined. Depending on the charges of the brane, this will allow some number of bulk cosmological constants to be adjusted to satisfy the Einstein equation.

\section{A generalized model}

We are interested in models where the bulk cosmological constant is piecewise constant, with each piece an integration constant that will be determined by the Einstein equation. The cosmological constant in the unimodular model is the same everywhere. This scenario is suitable for the two brane model, but is inadequate for the multibrane world when there are more than two branes. The model with $M$ types of 5-form field strengths is suitable for the $(M+1)$-brane model, in the uncompactified case, or the $M$-brane model, in the compactified case. However, it is not suitable for the $S^{1} / Z_{2}$ orbifold model, since the background 5 -form field strength must be set to zero due to the $\mathbb{Z}_{2}$ symmetry. As a consequence, the bulk cosmological constants are fixed, so it is useful to consider scenarios where the bulk cosmological constant can be piecewise adjustable. Here we present such a model, which is a generalization of the above models.

Let us first consider a model without branes, which includes a scalar field $\phi$ and its effective potential, $\Lambda(\phi)$ :

$$
S=\int d^{(d)} x \sqrt{|g|}\left\{\frac{1}{2 \kappa^{2}} R-\Lambda(\phi)-T^{a} \partial_{a} \phi\right\} .
$$

The independent variables of the model are $g_{a b}(x), T^{a}(x)$ and $\phi(x)$; the Einstein equation is

$$
G_{a b}=\kappa^{2}\left[-\Lambda(\phi)-T^{c} \partial_{c} \phi\right] g_{a b} .
$$

The variations of $S$ with respect to $T^{a}$ and $\phi$ give the following equations:

$$
\begin{aligned}
& \frac{\delta S}{\delta T^{a}}=0 \Rightarrow \sqrt{|g|} \partial_{a} \phi=0 \\
& \frac{\delta S}{\delta \phi}=0 \Rightarrow \partial_{a}\left(\sqrt{|g|} T^{a}\right)=\sqrt{|g|} \frac{\partial \Lambda}{\partial \phi} .
\end{aligned}
$$

Equation (25) implies that $\phi(x)=$ const, so that Eq. (24) becomes

$$
G_{a b}=-\Lambda(\phi=\text { const }) g_{a b}
$$

where $\Lambda(\phi)$ is an integration constant. Let us now consider special choices of $\Lambda(\phi)$. For example, if

$$
\Lambda(\phi)=\frac{1}{2} \phi^{2}
$$

the action $S$ reduces to the $d$-form field strength case we have already discussed [18]. To see this, define $T^{a}$ as the dual of a $(d-1)$ form $A_{(d-1)}$ :

$$
T^{a}=\frac{\epsilon^{a b_{1} \cdots b_{d-1}}}{(d-1) ! \sqrt{|g|}} A_{b_{1} \cdots b_{d-1}}
$$

Then Eq. (26) becomes

$$
\sqrt{|g|} \phi=\frac{\epsilon^{a b_{1} \cdots b_{d-1}}}{(d-1) !} \partial_{a} A_{b_{1} \cdots b_{d-1}}=\frac{\epsilon^{a_{1} \cdots a_{d}}}{d !} F_{a_{1} \cdots a_{d}}
$$

and substituting this back into the action (or equivalently, perform the functional integration over $\phi$ in the generating functional) and using $\left(\epsilon^{a_{1} \cdots a_{d} F_{a_{1}} \cdots a_{d}}\right)^{2}$ $=-(|g|) d ! F_{a_{1} \cdots a_{d}} F^{a_{1} \cdots a_{d}}$, gives

$$
S=\int d^{(d)} x \sqrt{|g|}\left\{\frac{1}{2 \kappa^{2}} R-\frac{1}{2 \cdot d !} F_{a_{1} \cdots a_{d}} F^{a_{1} \cdots a_{d}}\right\} .
$$

Alternatively, if we choose

$$
\Lambda(\phi)=\phi
$$

we recover the model of Ref. [17], which may be considered as a covariantized version of the unimodular gravity. The value of $\phi$ can be arbitrary in the orbifold model. We may also combine the two approaches to obtain an action $S$ :

$$
\begin{aligned}
S= & \int d^{(d)} x \sqrt{|g|}\left\{\frac{1}{2 \kappa^{2}} R-\frac{1}{2 \cdot d !}\right. \\
& \times F_{a_{1} \cdots a_{d}} F^{\left.a_{1} \cdots a_{d}-\phi-T^{a} \partial_{a} \phi\right\} .}
\end{aligned}
$$

We can easily further generalize this formalism to $M$ fields $T_{J}^{a}$ and $\phi_{J}, \quad J=1,2, \ldots, M$ and couple them to the $(d-2)$-branes: 


$$
\begin{aligned}
S= & \int d^{(d)} x \sqrt{|g|}\left\{\frac{1}{2 \kappa^{2}} R-\sum_{J=1}^{M} \phi_{J}+T_{J}^{a} \partial_{a} \phi_{J}\right\}+\sum_{i}-\frac{\sigma_{i}}{2} \int d^{(d-1)} \xi \sqrt{\left|\gamma_{n}\right|}\left[\gamma_{n}^{\mu \nu} \partial_{\mu} X^{a} \partial_{\nu} X^{b} g_{a b}\left(X_{n}\right)-C\right] \\
& +\sum_{i, J} \mu_{i}^{J} \int d^{(d-1)} \xi \sqrt{|g|} T_{J}^{a} \epsilon_{a b_{1} \cdots b_{d-1}} \partial_{\mu_{1}} X^{b_{1}} \cdots \partial_{\mu_{d-1}} X^{b_{d-1}} \epsilon^{\mu_{1} \cdots \mu_{d-1}} .
\end{aligned}
$$

If the $n$th 3 -brane has charges $\mu_{n}^{J}, J=1,2, \ldots, M$, then the resulting cosmological constant in the bulk between the $(n$ $-1)$ th and the $n$th branes is given by

$$
-\Lambda_{n}=\sum_{J} \phi_{n}^{J}=\sum_{J}\left(\phi^{J}+\mu_{0}^{J}+\cdots+\mu_{n-1}^{J}\right) .
$$

Again we require $\Lambda_{n}>0$ for AdS spaces between branes. There are $M$ background constants $\phi^{J}, J=1,2, \ldots, M$, that are integration constants to be determined. In this case, only the sum of $\phi$ 's is determined by the Einstein equation, so only one bulk cosmological constant can be adjusted to satisfy the Einstein equation. Here, $\Lambda(\phi)=\phi$ can be either positive or negative. We can combine one $\phi$ field with $M$ 4 -form potentials. We shall comment on the dynamics of this system when applied to the static multibrane solution.

\section{SOLUTION TO EINSTEIN EQUATION AND GENERAL FORMALISM}

We consider a multibrane world scenario. The objective is to provide a model which concurrently solves both traditional fine tunings, the hierarchy and the cosmological constant problems. We first give the general multibrane solution and the 4D effective action formalism.

\section{A. Multibrane world solution}

Let us first recall the setup of the static multibrane world. Consider $N$ parallel 3 -branes, located at $y=L_{i}, i=0, \ldots N$ -1 . The brane at $y=L_{i}$ has brane tension $\sigma_{i}$. Unless stated otherwise, the brane tensions are taken to be positive, except for branes at orbifold fixed points, for which we allow the possibility of negative brane tensions. In the uncompactified case, there are $N+1$ AdS bulk spaces, with 5D energymomentum tensor $T_{a b}=\Lambda_{i} g_{a b}$ in the bulk between the ( $i$ $-1)$ th and the $i$ th branes. We start with the metric

$$
d s^{2}=d y^{2}+A(y)\left[-d t^{2}+\exp (2 H t) \delta_{i j} d x^{i} d x^{j}\right] .
$$

Note that a rescaling of $\left(t, x^{i}\right)$ rescales both $A(y)$ and $H$, but leaves $H^{2} / A(y)$ invariant, so we have the freedom to fix the overall normalization of $A(y)$. The $G_{05}$ component of the Einstein's equation $G_{a b}=\kappa^{2} T_{a b}=8 \pi G_{5} T_{a b}$ [see for example Eq. (21)] is satisfied trivially, while the $G_{00}$ and the $G_{55}$ components give, respectively,

$$
\begin{aligned}
\frac{A^{\prime \prime}}{A}= & \frac{2 H^{2}}{A}+\frac{2 \kappa^{2}}{3}\left[\sum_{i=0}^{N} \Lambda_{i} \Theta\left(y-L_{i-1}\right) \Theta\left(L_{i}-y\right)\right. \\
& \left.-\sum_{i=0}^{N-1} \sigma_{i} \delta\left(y-L_{i}\right)\right] \\
\left(\frac{A^{\prime}}{A}\right)^{2}= & \frac{4 H^{2}}{A}+\frac{2 \kappa^{2}}{3}\left[\sum_{i=0}^{N} \Lambda_{i} \Theta\left(y-L_{i-1}\right) \Theta\left(L_{i}-y\right)\right]
\end{aligned}
$$

(where $-L_{-1}=L_{N}=\infty$ in the uncompactified case). The $G_{i j}$ components do not yield any additional equations. It is convenient to define $q_{i} \equiv \kappa^{2} \sigma_{i} / 3$ and $k_{i} \equiv \sqrt{\kappa^{2} \Lambda_{i} / 6}$, where both have mass dimension 1 .

Integrating the $G_{00}$ component of Einstein's equation across the $i$ th brane yields the Israel jump condition [24]:

$$
\lim _{y \rightarrow L_{i}+}\left(\frac{A^{\prime}}{A}\right)-\lim _{y \rightarrow L_{i}}\left(\frac{A^{\prime}}{A}\right)=-\frac{2 \kappa^{2} \sigma_{i}}{3} \equiv-2 q_{i} .
$$

In general, there is also an equation of motion for each brane; these are discussed for the 5-form field strength case in Appendix A. For stationary branes only the 5 th component of the equation of motion for the embedding coordinates is nontrivial. Since the derivatives of the metric at the brane are discontinuous, we have to average over the two sides of the brane. In the above metric ansatz, the equation for the $i$ th brane reduces to

$$
\sigma_{i}\left[\lim _{y \rightarrow L_{i}+}\left(\frac{A^{\prime}}{A}\right)+\lim _{y \rightarrow L_{i}-}\left(\frac{A^{\prime}}{A}\right)\right]=\Lambda_{i+1}-\Lambda_{i}
$$

(see Appendix A for details). In the 5-form field strength model with $M$ 5-form field strengths, Eq. (22) gives

$$
-\frac{1}{2} \sum_{J} e_{i}^{J}\left(e_{(i)}^{J}+e_{(i+1)}^{J}\right)=\Lambda_{i+1}-\Lambda_{i} .
$$

This means that, in the stationary situation, the average of $A^{\prime} / A$ of each brane is simply the difference of the bulk cosmological constants on its two sides. In the symmetric case, i.e., $\Lambda_{i+1}=\Lambda_{i}$, the limiting value of $A^{\prime} / A$ from the two sides are equal in magnitude but have opposite signs, as expected. Equation (39) suggests that equations analogous to Eq. (40) ought to hold for other models in which the bulk cosmological constant emerges as an integration constant. This is indeed the case for the generalized model (34), where 
(a)

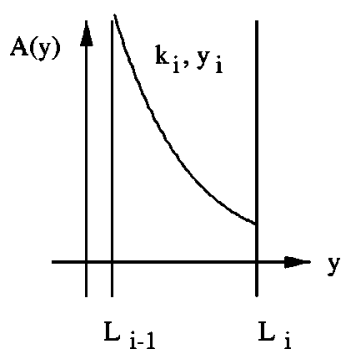

(b)

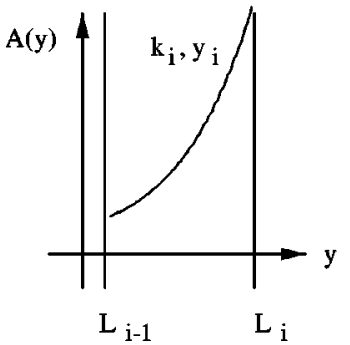

(c)

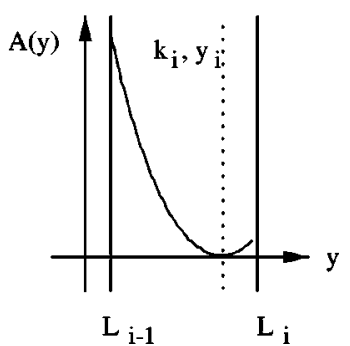

FIG. 2. Schematic diagram of the possible types of behavior of the metric coefficient $A(y)$ in the bulk between the branes.

$H\left(L_{i}\right)= \pm \frac{k_{i}}{\sinh \left[k_{i}\left(L_{i}-y_{i}\right)\right]}$.

This completes the setup of the problem. Not surprisingly, solutions of the Einstein equation, Eq. (37), for stationary branes automatically satisfy the brane equations of motion, Eq. (39), when the jump conditions Eq. (38) are imposed. The approach we shall take is to first solve the Einstein equation (37) in the bulk, then impose the jump condition (38), and finally show that the brane Eq. (39) of motion (for each brane) is automatically satisfied.

Similar systems have been studied in Ref. [25]. Defining $k_{i} \equiv \sqrt{\kappa^{2} \Lambda_{i} / 6}$, the solution to Einstein's equation (37) in the bulks is

$$
A(y)=\frac{H^{2} \sinh ^{2}\left[k_{i}\left(y-y_{i}\right)\right]}{k_{i}^{2}} \quad\left(L_{i-1}<y<L_{i}\right)
$$

(as before $-L_{-1}=L_{N}=\infty$ in the uncompactified case). Continuity of the metric at each of the branes imposes the constraints,

$$
\frac{\sinh ^{2}\left[k_{i}\left(L_{i}-y_{i}\right)\right]}{k_{i}^{2}}=\frac{\sinh ^{2}\left[k_{i+1}\left(L_{i}-y_{i+1}\right)\right]}{k_{i+1}^{2}},
$$

and the jump condition Eq. (38) gives

$$
q_{i}=\frac{k_{i} \cosh \left[k_{i}\left(L_{i}-y_{i}\right)\right]}{\sinh \left[k_{i}\left(L_{i}-y_{i}\right)\right]}-\frac{k_{i+1} \cosh \left[k_{i+1}\left(L_{i}-y_{i+1}\right)\right]}{\sinh \left[k_{i+1}\left(L_{i}-y_{i+1}\right)\right]} .
$$

It is easy to check, using both the continuity and the jump equations, that the brane equation of motion (39) is automatically satisfied for each brane. The 4D cosmological constant as seen by observers on the $i$ th brane is

$$
H^{2}\left(L_{i}\right) \equiv \frac{H^{2}}{A\left(L_{i}\right)}=\frac{k_{i}^{2}}{\sinh ^{2}\left[k_{i}\left(L_{i}-y_{i}\right)\right]},
$$

where $A\left(L_{i}\right)$ is the value of the warp factor at the $i$ th brane, i.e., $A\left(L_{i}\right)=A\left(y=L_{i}\right)$. Hence there is an inherent sign ambiguity in the definition of $H\left(L_{i}\right)$ :

Since $k_{i}>0$ by definition and we assume $H\left(L_{i}\right)>0$, the sign we must choose is determined by the $\operatorname{sign}$ of $\sinh \left[k_{i}\left(L_{i}\right.\right.$ $\left.-y_{i}\right)$, or, equivalently, the sign $s_{i}^{-}$of $A^{\prime}$ at $y \rightarrow L_{i}^{-}$. Inverting this expression, we can express the separation between two consecutive branes, $\Delta l_{i} \equiv k_{i}\left(L_{i}-L_{i-1}\right)$, in terms of the Hubble constants on those branes:

$$
\Delta l_{i}=-s_{i-1}^{+} \sinh ^{-1}\left(\frac{k_{i}}{H\left(L_{i-1}\right)}\right)+s_{i}^{-} \sinh ^{-1}\left(\frac{k_{i}}{H\left(L_{i}\right)}\right)
$$

where $s_{i-1}^{+}$is the sign of $A^{\prime}$ at $y \rightarrow L_{i-1}^{+}$.

The metric coefficient $A(y)$ can have three different types of behaviors in the bulk between the branes, as illustrated in Fig. 2. We see from Eq. (42) that $A(y)$ may decrease monotonically, increase monotonically, or must vanish at an intermediate $y$, which corresponds to a coordinate singularity of the metric, Eq. (36), a particle horizon. Such horizons are discussed further in Sec. VII.

Because the definition of $\Delta l_{i}$ incorporates both the brane separation and $k_{i}$, these equations can be thought of as determining $\left(L_{i}-L_{i-1}\right)$ from $H\left(L_{i}\right)$ and $H\left(L_{i-1}\right)$ for given $k_{i}$, or as determining $k_{i}$ from $H\left(L_{i}\right)$ and $H\left(L_{i+1}\right)$, with the brane separations governed by some higher energy dynamics. In terms of the bulk cosmological constants on either side of the $i$ th brane,

$$
H^{2}\left(L_{i}\right)=\frac{\left[q_{i}^{2}-\left(k_{i+1}+k_{i}\right)^{2}\right]\left[q_{i}^{2}-\left(k_{i+1}-k_{i}\right)^{2}\right]}{4 q_{i}^{2}},
$$

in agreement with Ref. [26]. Note that Eq. (48) appears to give the expansion rate on the $i$ th brane in terms of local quantities - the bulk cosmological constants on either side of the brane, and its tension-but in the context of a full multibrane spacetime, these quantities may depend on the positions of the other branes, and the locations of particle horizons, which are not necessarily nearby. The form of the metric in Eq. (36) assumes a constant $H$, but when matter density $\rho$ on the brane is included, one can easily solve for $H^{2}$ on a particular brane without solving for the metric in all regions of spacetime $[9,27,28,26]$. The result is simple: one 
simply replaces $q_{i} \rightarrow q_{i}+\kappa^{2} \rho / 3$. This result provides a determination of the 4D Newton's constant $G_{N}$ by requiring $H^{2}$ to have the standard form [9]

$$
H^{2} \approx 8 \pi G_{N}\left(\Lambda_{e f f}+\rho+\cdots\right) / 3 .
$$

As we shall see later, this way of determining $G_{N}$ is valid only when $A(y)$ is peaked at the particular brane where the expansion rate is evaluated.

\section{B. The 4D effective action}

Consider the 5D action $\mathbf{S}^{(5)}$ for the 5D gravity plus scalar fields confined on branes:

$$
\begin{aligned}
\mathbf{S}^{(5)}= & \int d y d^{4} x \sqrt{-g}\left[\frac{R^{(5)}}{2 \kappa^{2}}+\Lambda+\sum_{i} \delta\left(y-L_{i}\right)\right. \\
& \left.\times \sqrt{g^{55}}\left(-\sigma_{i}+\mathbf{L}_{i}\right)\right]+\int_{\Sigma} \frac{K \sqrt{-\gamma}}{\kappa^{2}} d^{4} x
\end{aligned}
$$

where the bulk cosmological constant $\Lambda$ ( $\Lambda>0$ for AdS) is piecewise constant, $\sigma_{i}$ is the $i$ th brane tension, the surface term is the Hartle-Hawking term, needed for theories with boundaries, and

$$
\mathbf{L}_{i}=\frac{1}{2} g^{\mu \nu} \phi_{i, \mu} \phi_{i, \nu}-\frac{1}{2} M_{H i}^{2} \phi_{i}^{2}-\lambda_{i} \phi_{i}^{4}-\frac{\phi_{i}^{6}}{M_{c i}^{2}}+\cdots
$$

where the scalar field $\phi_{i}$ is confined to the brane at $L_{i}$. Recall that $8 \pi G_{5}=\kappa^{2}=8 \pi / M^{3}$. Generically, the scalar field masses $M_{H i}$ (which are real after spontaneous symmetry breaking) and the cutoff masses $M_{c i}$ are expected to be comparable to $M$ in order of magnitude. The characteristic scales brane tensions and the bulk cosmological constant are also expected to be set by $M$, i.e., $\sigma_{i} \sim M^{4}$ while $\Lambda \sim M^{5}$. The couplings $\lambda_{i}$ are of order unity.

Following Ref. [7], we can integrate out the $y$ direction to obtain an effective 4D theory. As we shall discuss in more detail, the zeros of the metric $A(y)$ are particle horizons. That is, for an observer on a brane, it takes infinite time for a light-like signal to travel from the brane to the particle horizon. This implies that the integration over $y$ is only over the region between particle horizons that includes the position $L$ of the visible brane. We can decompose the $\mu \nu$ components of the 5D Ricci tensor $R_{\mu \nu}^{(5)}$ into the 4D Ricci tensor $R_{\mu \nu}^{(4)}\left(\hat{\gamma}_{\mu \nu}\right)$ and the extrinsic curvature $K_{\mu \nu}$ (and $K$ $\left.=g^{\mu \nu} K_{\mu \nu}\right)$ :

$$
R_{\mu \nu}^{(5)}=R_{\mu \nu}^{(4)}-g^{55} \partial_{y} K_{\mu \nu}-g^{55} K_{\mu \nu} K+2 g^{55} K_{\mu}^{\lambda} K_{\lambda \nu}
$$

where we recall the $5 \mathrm{D}$ metric ${ }^{4}$

$$
d s^{2}=A(y) \hat{\gamma}_{\mu \nu}(x) d x^{\mu} d x^{\nu}+d y^{2}
$$

so that the pullback on the $L_{i}$ brane $\gamma_{\mu \nu}=g_{\mu \nu}=A(y$ $\left.=L_{i}\right) \hat{\gamma}_{\mu \nu}(x)$, and $\sqrt{-g}=A^{2}(y) \sqrt{-\hat{\gamma}}$. Using the fact that $K_{\mu \nu}=g_{\mu \nu} A^{\prime} / 2 A$ (where the prime indicates derivative with respect to $y$ ) and $K=2 A^{\prime} / A$, this gives

$$
\begin{aligned}
\sqrt{-g} R^{(5)}= & \sqrt{-\hat{\gamma}}\left[A(y) R^{(4)}-g^{\mu \nu} \partial_{y} K_{\mu \nu}\right. \\
& \left.-K^{2}+2 K^{\lambda \rho} K_{\lambda \rho}+R_{55}\right] \\
= & \sqrt{-\hat{\gamma}}\left[A(y) R^{(4)}-\left(A(y)^{\prime}\right)^{2}-4 A(y)^{\prime \prime} A(y)\right] .
\end{aligned}
$$

We substitute $A(y)$ and its derivatives into the $5 \mathrm{D}$ action $\mathbf{S}^{(5)}$ and integrate over $y$ to obtain the 4D low energy effective theory. This "integrating out" of the 5 th dimension in $\mathbf{S}^{(5)}$ yields the effective 4D action $\mathbf{S}^{(4)}$ :

$$
\begin{aligned}
\mathbf{S}^{(4)}= & \int d^{4} x \sqrt{-\hat{\gamma}} R^{(4)} / 2 \kappa_{N}^{2}-\int d^{4} x \sqrt{-\hat{\gamma}} \Lambda_{e f f} \\
& +\sum_{i} \int d^{4} x A^{2}\left(L_{i}\right) \sqrt{-\hat{\gamma}}\left[\frac{1}{2 A\left(L_{i}\right)} \hat{\gamma}^{\mu \nu} \phi_{i, \mu} \phi_{i, \nu}\right. \\
& \left.-\frac{1}{2} M_{H}^{2} \phi_{i}^{2}-\lambda_{i} \phi_{i}^{4}-\frac{\phi_{i}^{6}}{M_{c i}^{2}}+\cdots\right] .
\end{aligned}
$$

We see that the $4 \mathrm{D}$ gravitational coupling is given by

$$
\frac{1}{2 \kappa_{N}^{2}}=\frac{1}{2 \kappa^{2}} \int A(y) d y .
$$

Suppose that $A(y)$ is peaked at the $i$ th brane, namely the Planck brane, it is convenient to fix the normalization $A\left(L_{i}\right)=1$; then, to a good approximation, we have

$$
G_{N} \simeq G_{5} \frac{2 k_{i} k_{i+1}}{k_{i}+k_{i+1}}
$$

${ }^{4} \mathrm{~A}$ more general ansatz for the $5 \mathrm{D}$ metric is

$$
d s^{2}=A[y \Phi(x)] \hat{\gamma}_{\mu \nu}(x) d x^{\mu} d x^{\nu}+\Phi(x)^{2} d y^{2}
$$

where $\Phi(x)$ is a scalar field and it is assumed that the metric $\hat{\gamma}_{\mu \nu}$ and field $\Phi$ vary on lengthscales and timescales large compared to $1 / k_{i}$. This ansatz would yield additional scalar fields in the 4D effective action (59) below corresponding to the location of the different branes. These additional scalar fields are related to the stability issue discussed in the second last paragraph of the introduction. We will ignore these fields in this paper, since in all brane models one needs to appeal to a stabilization mechanism such as the Goldberger-Wise mechanism to stabilize the distance between the branes. 


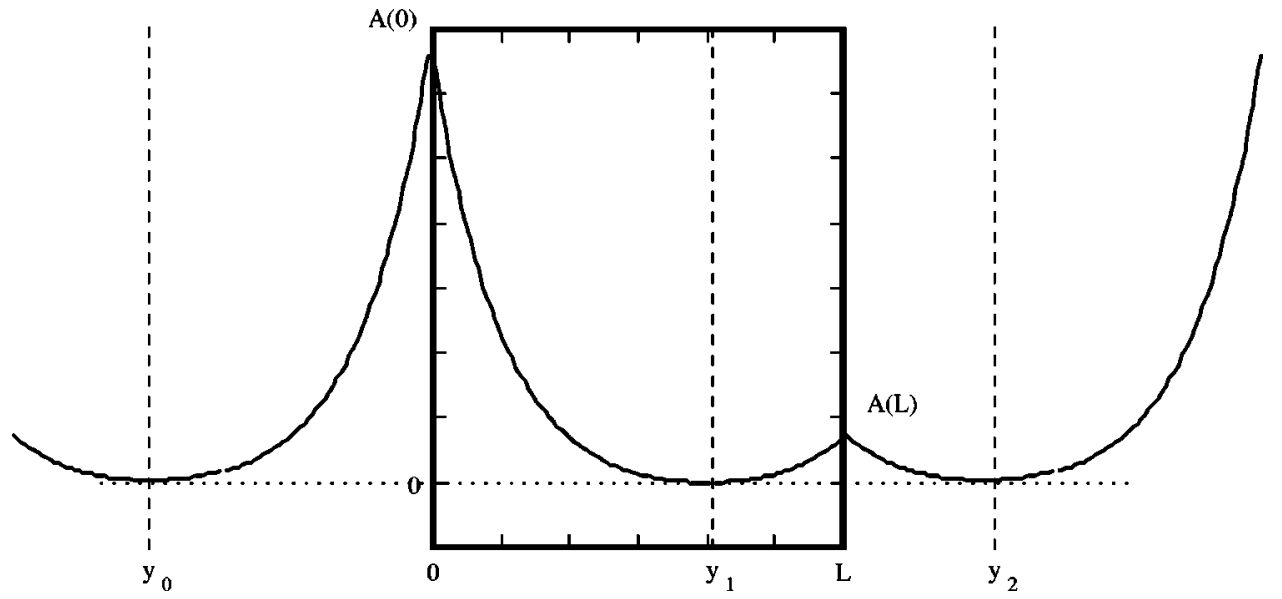

FIG. 3. The two brane model in the uncompactified case, with the $q_{0}$ brane at $y=0$ and the $q_{1}$ brane at $y=L$. In the $S^{1} / Z_{2}$ orbifold version (boxed), the two branes sit at the two fixed points. The metric factor $A(y)$ is shown schematically. for the region between particle horizons that includes the $i$ th brane, and $8 \pi G_{5}=\kappa^{2}$. Collecting all the contributions to the effective cosmological constant $\Lambda_{e f f}$, we have

$$
\begin{aligned}
\Lambda_{e f f}= & \int\left[-\Lambda A(y)^{2}+\left(\left[A(y)^{\prime}\right]^{2}+4 A(y)^{\prime \prime} A(y)\right) / 2 \kappa^{2}\right] d y \\
& -2 A(y)^{\prime} A(y) /\left.\kappa^{2}\right|_{\text {boundaries }}+\sum_{i} A^{2}\left(L_{i}\right) \sigma_{i} \\
= & -\int \Lambda A(y)^{2} d y-\frac{3}{2 \kappa^{2}} \int\left[A^{\prime}(y)\right]^{2} d y \\
& +\sum_{i} A^{2}\left(L_{i}\right) \sigma_{i}
\end{aligned}
$$

where $A^{\prime \prime}$ is singular at the branes. An integration by parts on the $A^{\prime \prime} A$ term removes the Hartle-Hawking boundary term, as expected. The integration over $y$ is between particle horizons and $\Lambda$ is piecewise constant. Besides the contributions from the brane tensions and the bulk $\Lambda$, we see that there is a contribution from the 5D Ricci scalar $R^{(5)}$ to $\Lambda_{e f f}$. We can now re-express $k_{i}$ (or $\Lambda$ ) and $y_{i}$ in $\Lambda_{e f f}$ in terms of the brane tensions $q_{i}$ (or $\sigma_{i}$ ) and the brane separations $L_{i}$. A priori, we expect $\Lambda_{e f f}$ to be of order Planck scale, but for large brane separations, we shall see that the boundary conditions at the branes fix the piecewise constant bulk $\Lambda$ so that an almost exact cancellation among the terms in Eq. (58) renders $\Lambda_{\text {eff }}$ exponentially small.

We now redefine the field $\hat{\phi}_{i}=\sqrt{A\left(L_{i}\right)} \phi_{i}$ to absorb the factor $A\left(L_{i}\right)$ in the kinetic term of the Lagrangian $\mathbf{L}_{i}$, so the effective 4D low energy action becomes

$$
\begin{aligned}
\mathbf{S}^{(4)}= & \int d^{4} x \sqrt{-\hat{\gamma}}\left[\frac{R^{(4)}}{2 \kappa_{N}^{2}}-\Lambda_{e f f}+\mathbf{L}_{C F T}+\sum_{i}\left(\frac{1}{2} \hat{\gamma}^{\mu \nu} \hat{\boldsymbol{\phi}}_{i, \mu} \hat{\boldsymbol{\phi}}_{i, \nu}\right.\right. \\
& \left.\left.-\frac{m_{H i}^{2} \hat{\boldsymbol{\phi}}_{i}^{2}}{2}-\lambda_{i} \hat{\boldsymbol{\phi}}_{i}^{4}-\frac{\hat{\phi}_{i}^{6}}{m_{c i}^{2}}+\cdots\right)\right]
\end{aligned}
$$

where $m_{H i}^{2}=A\left(L_{i}\right) M_{H i}^{2}$ and $m_{c i}^{2}=A\left(L_{i}\right) M_{c i}^{2}$. It is important to note that the Newton's constant and the effective cosmological constant $\Lambda_{e f f}$ are the same everywhere, so observers on the Planck (hidden) brane see the same $G_{N}$ and $\Lambda_{\text {eff }}$ as observers on the $\mathrm{TeV}$ (visible) brane, even if the visible brane tension is negative, as is the case in the RandallSundrum model. To be more realistic, $\mathbf{L}_{v i s i b l e}$ may be replaced by the standard model Lagrangian density. In $\mathbf{S}^{(4)}$, we have also included a conformal field theory term $\mathbf{L}_{C F T}$. Using the AdS-CFT correspondence [10], the effect of the Kaluza-Klein (KK) gravity modes may be incorporated into a conformal field theory on the brane. This should be the case when the model covers the region between particle horizons, where the gravity $\mathrm{KK}$ modes have a continuous mass spectrum. In the orbifold model, where the gravity $\mathrm{KK}$ modes may be discrete, in which case $\mathbf{L}_{C F T}$ should be replaced by another appropriate strongly interacting field theory.

Note that the warp factor $A(y)$ does not appear in the 4D effective action. Although we have chosen the warp factor at the Planck brane to be one, we could have chosen the warp factor at the visible brane to be one instead. The physics depends only on the ratio of warp factors on the two branes, which is unaltered by this rescaling. However, if we choose $A\left(L_{\text {visible }}\right)=1$, then the electroweak scale should be taken to be the fundamental scale, while the Planck mass is a derived quantity.

\section{TWO BRANE WORLD}

To render the general analysis more transparent, consider the simplest case, a two brane model (see Fig. 3). Setting $L_{0}=0, L_{1}=L$, the solution for the bulks outside the branes is

$$
\begin{array}{cc}
A(y)=\frac{H^{2} \sinh ^{2}\left[k_{0}\left(y-y_{0}\right)\right]}{k_{0}^{2}} \quad(y<0) & \quad(0<y<L) \\
A(y)=\frac{H^{2} \sinh ^{2}\left[k_{1}\left(y-y_{1}\right)\right]}{k_{1}^{2}} \quad(y>L), &
\end{array}
$$

where $y_{0}, y_{1}$ and $y_{2}$ are constants, and give the locations of the zeros of $A(y)$ when they are included in the domain of the spacetime in the fifth dimension. 
Continuity of the metric at the branes implies that

$$
\begin{gathered}
\frac{\sinh ^{2}\left(k_{0} y_{0}\right)}{k_{0}^{2}}=\frac{\sinh ^{2}\left(k_{1} y_{1}\right)}{k_{1}^{2}} \\
\frac{\sinh ^{2}\left[k_{1}\left(L-y_{1}\right)\right]}{k_{1}^{2}}=\frac{\sinh ^{2}\left[k_{2}\left(L-y_{2}\right)\right]}{k_{2}^{2}} .
\end{gathered}
$$

The jump conditions at the two branes are

$$
\begin{gathered}
-\frac{k_{0} \cosh \left(k_{0} y_{0}\right)}{\sinh \left(k_{0} y_{0}\right)}+\frac{k_{1} \cosh \left(k_{1} y_{1}\right)}{\sinh \left(k_{1} y_{1}\right)}=q_{0} \\
\frac{k_{1} \cosh \left[k_{1}\left(L-y_{1}\right)\right]}{\sinh \left[k_{1}\left(L-y_{1}\right)\right]}-\frac{k_{2} \cosh \left[k_{2}\left(L-y_{2}\right)\right]}{\sinh \left[k_{2}\left(L-y_{2}\right)\right]}=q_{1} .
\end{gathered}
$$

The expansion rate seen by observers on the brane at $y$ $=0$ is $H(0)=H / \sqrt{A(0)}$, where

$$
\begin{aligned}
\frac{H^{2}}{A(0)} & =\frac{k_{0}^{2}}{\sinh ^{2}\left(k_{0} y_{0}\right)} \\
& =\frac{k_{1}^{2}}{\sinh ^{2}\left(k_{1} y_{1}\right)} \\
& =\frac{\left[k_{1}^{2}-\left(k_{0}+q_{0}\right)^{2}\right]\left[k_{1}^{2}-\left(k_{0}-q_{0}\right)^{2}\right]}{4 q_{0}^{2}},
\end{aligned}
$$

with $k_{1}^{2}-\left(k_{0} \pm q_{0}\right)^{2}>0$ or $<0$, in agreement with Ref. [26], which uses a slightly different approach. Similarly, the expansion rate seen by observers on the brane at $y=L$ is $H(L)=H / \sqrt{A(L)}$, where

$$
\begin{aligned}
\frac{H^{2}}{A(L)} & =\frac{k_{2}^{2}}{\sinh ^{2}\left[k_{2}\left(L-y_{2}\right)\right]} \\
& =\frac{k_{1}^{2}}{\sinh ^{2}\left[k_{1}\left(L-y_{1}\right)\right]} \\
& =\frac{\left[k_{1}^{2}-\left(k_{2}+q_{1}\right)^{2}\right]\left[k_{1}^{2}-\left(k_{2}-q_{1}\right)^{2}\right]}{4 q_{1}^{2}},
\end{aligned}
$$

with $k_{1}^{2}-\left(k_{2} \pm q_{1}\right)^{2}>0$ or $<0$. We can rescale $t$ so that $A(0)=1$, and the Hubble constants on the two branes are, respectively, $H(0)=H$ and $H(L)=H / \sqrt{A(L)}$. Note that although Eqs. (63) and (64) appear to determine the expansion rates on the two branes completely in terms of local quantities (i.e., the local brane tensions, and bulk cosmological constants just outside each brane), the values of these quantities on or near the two branes are connected via $k_{1}$ and $y_{1}$.

The 4D Newton's constant $G_{N}$ can be determined by introducing a small matter density $\rho$ to the visible brane, that is, $q_{0} \rightarrow q_{0}+\kappa^{2} \rho / 3$, finding the Hubble constant $H$, as in Eq. (49) and Refs. [9,26-28], and then requiring that $H^{2}$ $=\left(8 \pi G_{N} / 3\right)\left(\Lambda_{e f f}+\rho\right)$; the result is

$$
4 \pi G_{N} q_{0}=\kappa^{2} \alpha_{0} k_{0}\left[1+2 \kappa^{2} \Lambda_{e f f}\left(2 \alpha_{0}+k_{0}\right) / 3 q_{0} k_{0}\right]
$$

where $\alpha_{0} \equiv q_{0}-k_{0}$. Although $L$ dependent, the correction term is small if $G_{N} \Lambda_{e f f} / k_{0}^{2} \ll 1$, which is the case here, so, to a very good approximation, we have

$$
G_{N}=\frac{\kappa^{2} \alpha_{0} k_{0}}{4 \pi q_{0}}
$$

Positivity of $G_{N}$ requires $\alpha_{0}>0$; to be specific, let us consider

$$
0 \leqslant k_{1} \leqslant q_{0}-k_{0}
$$

Since the expansion rates $H$ and $H(L)$ given in Eqs. (63) and (64) depend on $k_{1}$, our goal is to express $H$ and $H(L)$ as functions of $L$ and the parameters $k_{0}, k_{2}, q_{0}$ and $q_{1}$. This requires an expression relating $L$ and $k_{1}$; from Eqs. (61) and (62) we find

$$
\begin{aligned}
k_{1} L= & \sinh ^{-1}\left(\frac{k_{1}}{H(0)}\right)+\sinh ^{-1}\left(\frac{k_{1}}{H(L)}\right) \\
= & \sinh ^{-1}\left(\frac{2 k_{1} q_{0}}{\sqrt{\left[k_{1}^{2}-\left(k_{0}+q_{0}\right)^{2}\right]\left[k_{1}^{2}-\left(k_{0}-q_{0}\right)^{2}\right]}}\right) \\
& +\sinh ^{-1}\left(\frac{2 k_{1} q_{1}}{\sqrt{\left[k_{1}^{2}-\left(k_{2}+q_{1}\right)^{2}\right]\left[k_{1}^{2}-\left(k_{2}-q_{1}\right)^{2}\right]}}\right) .
\end{aligned}
$$

Here, Eq. (68) is regarded as a relation that determines $k_{1}$ in terms of $L, q_{0}, q_{1}, k_{0}$ and $k_{2}$. In the 5 -form field strength model, $k_{1}, k_{0}$ and $k_{2}$ will adjust together as the background field strength is determined as a function of $L, q_{0}$ and $q_{1}$. In general, $H(0) \neq H(L)$. Because of the condition (67), $H$ $\rightarrow 0$ as $k_{1} \rightarrow \alpha_{0}=q_{0}-k_{0}$ from below. This means that the expansion rate as seen by observers on the visible brane becomes exponentially small for large $L$,

$$
H^{2} \approx 4 \alpha_{0}^{2} e^{-2\left(\alpha_{0} L-C\right)}
$$

where $\alpha_{0}>0$, and $\sinh (C)=k_{1} / H(L)$. As $k_{1} \rightarrow \alpha_{0}$ from below, $H(L)$ approaches a $L$-independent constant given by Eq. (64) and so does $C$. This implies that $\Lambda_{e f f}$ becomes exponentially small as $L$ increases.

Note that there is only one integration constant for the bulk cosmological constants. Consider the model of Eq. (34). Here $k_{0}^{2}=-\kappa^{2} \phi / 6, k_{1}^{2}=-\kappa^{2}\left(\phi+\mu_{0}\right) / 6$ and $k_{2}^{2}=-\kappa^{2}(\phi$ $\left.+\mu_{0}+\mu_{1}\right) / 6$, where the integration constant $\phi$ is negative and the brane charges $\mu_{0}$ and $\mu_{1}$ are essentially arbitrary constants.

In the symmetric case, where $k_{0}=k_{2}=k$ and $q_{0}=q_{1}=q$ (and let $\alpha=q-k=\alpha_{0}$ ), we have $H(L)=H(0)=H$, and $y_{1}$ $=-y_{0}=y_{2}=L / 2$. The constant $C$ in Eq. (69) becomes $C$ $=\alpha L / 2$, so, including a matter density $\rho$ (which is treated as a perturbation),

$$
H^{2} \approx 4 \alpha^{2} e^{-\alpha L}+\frac{2 \kappa^{2} \alpha k}{3 q_{0}} \rho .
$$


Thus, $\Lambda_{e f f}$ still decreases exponentially with $L$, but slower than in the nonsymmetric case.

\section{The $S^{1} / Z_{2}$ orbifold model}

To this point, we have concentrated on spacetimes that are noncompact in $y$, but similar results can be derived for the compactified case. First, we may choose to identify $k_{0}=k_{2}$ $=k_{1}$ and derive $k_{1}$ and $H$ in terms of $L$. Next, we can compactify the $y$ direction to a circle $S^{1}$ of length $2 L$. Placing the branes at $y=0$ and $y=L$, we may identify the two sides of $S^{1}$ to obtain a line segment. That is, we perform a $\mathbb{Z}_{2}$ orbifold, with one brane sitting at each of the two fixed points $(y=0, L)$. This $S^{1} / Z_{2}$ orbifold model is particularly simple, since there is only one bulk space between the branes sitting at the two end points. This may be considered as an expanding (non-supersymmetric) version of the Horava-Witten model [29], and is discussed in Ref. [4]. The model has branes with tension $\sigma_{0}=3 q_{0} / \kappa^{2}$ at $y=0$ and $\sigma_{L}=3 q_{1} / \kappa^{2}$ at $y=L$, separated by AdS space with bulk cosmological constant $-6 k^{2} / \kappa^{2}$, which is treated as an integration constant. The solution is

$$
A(y)=\frac{H^{2}}{k^{2}} \sinh ^{2}\left[k\left(y-y_{0}\right)\right]
$$

for $L>y>0$. Because of the orbifold symmetry of the model, the jump conditions are

$$
\begin{aligned}
& 2 k / q_{0}=\tanh k y_{0} \\
& 2 k / q_{1}=\tanh \left[k\left(L-y_{0}\right)\right]
\end{aligned}
$$

at $y=0$ and $L$, respectively. Combining the jump conditions implies

$$
\frac{q_{0}}{2 k}=\frac{\tanh k L-q_{1} / 2 k}{1-\left(q_{1} / 2 k\right) \tanh k L}
$$

if $q_{1} / 2 k= \pm 1$, then $q_{0} / 2 k=\mp 1$ irrespective of $k L$, but for $q_{1} / 2 k \neq 1, q_{0} / 2 k \rightarrow 1$ as $k L \rightarrow \infty$. According to our viewpoint, Eq. (73) determines $k$ given $q_{0}, q_{1}$ and $L$.

The expansion rates on the branes are $H(0)=H / \sqrt{A(0)}$ and $H(L)=H / \sqrt{A(L)}$, where $H^{2}(0)=k^{2}\left[\left(q_{0} / 2 k\right)^{2}-1\right]$ and $H^{2}(L)=k^{2}\left[\left(q_{1} / 2 k\right)^{2}-1\right]$. We see that $L$ is related to $k$ via

$$
L=\frac{1}{2 k} \ln \left[\frac{\left(1+2 k / q_{0}\right)\left(1+2 k / q_{1}\right)}{\left(1-2 k / q_{0}\right)\left(1-2 k / q_{1}\right)}\right] .
$$

Eliminating $k$, we find that

$H^{2}(0)=\frac{q_{0}^{2}}{4}\left[\frac{\left(q_{0}+q_{1}\right)^{2}-2 q_{0} q_{1} t^{2}-2 q_{1}^{2} t^{2}+\left(q_{0}+q_{1}\right) \Delta}{\left(q_{0}+q_{1}\right)^{2}-2 q_{0} q_{1} t^{2}+\left(q_{0}+q_{1}\right) \Delta}\right]$

where $t \equiv \tanh k L$ and $\Delta \equiv \sqrt{\left(q_{0}+q_{1}\right)^{2}-4 q_{0} q_{1} t^{2}}$.

For large values of $q_{0} L$, this reduces to

$$
H^{2}(0) \approx \frac{q_{0}^{2}\left(q_{1}+q_{0}\right)}{\left(q_{1}-q_{0}\right)} e^{-q_{0} L}
$$

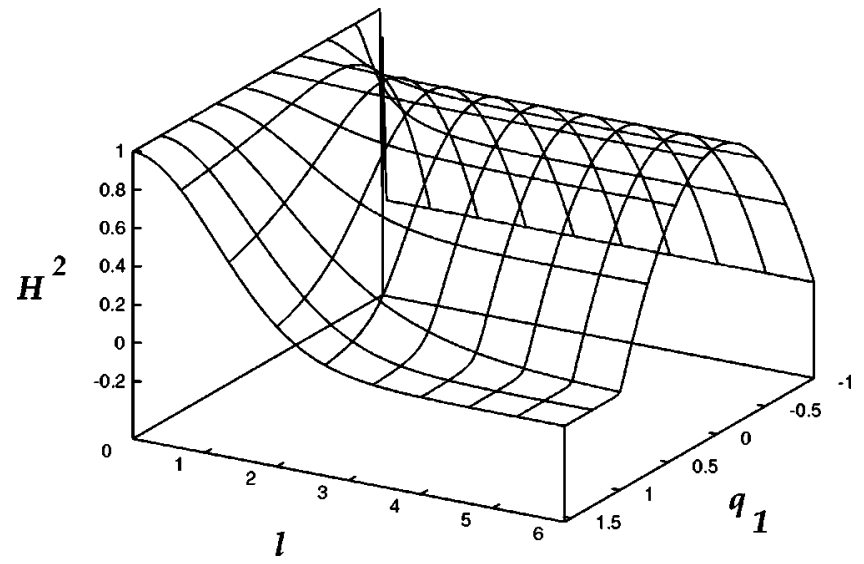

FIG. 4. Hubble constant $H^{2}$ on the brane at $L_{0}=0$ as a function of the dimensionless separation $l=k L$ and the hidden brane tension $q_{1}$, in units such that the observable brane tension $q_{0}=1$. Note that $H^{2}=0$ for $q_{1}=-1$, the RS scenario.

where $k \simeq q_{0}\left(1-e^{-q_{0} L}\right) / 2$. Thus, for $q_{0} L \gg 1$ and $\left|q_{1}\right|>q_{0}$ $>0$, the cosmological constant on the $y=0$ brane is positive, and exponentially small. Moreover, although Eq. (76) may appear singular as $q_{1} \rightarrow q_{0}$ in fact

$$
H^{2}(0) \approx q_{0}^{2} e^{-q_{0} L / 2}
$$

in that case.

The behavior of Eq. (75) is shown in Fig. 4 where we have taken $q_{0}=1$ in some units. For $q_{1}>q_{0}$ (i.e., $q_{1}>1$ ), $H^{2}=H^{2}(0)$ brane becomes exponentially small for large $l$ $=k L$. As $q_{1}$ decreases toward $q_{0}=1, H^{2}$ still drops off exponentially, but at a slower rate. For still smaller $q_{L}, H^{2}$ goes to a constant $\sim 1$ for large $l$ implying a large expansion rate on the $L=0$ brane. However, $H_{L}^{2}=H^{2}(L)$ then becomes exponentially small, as the roles of the branes at $y=0$ and $L$ reverse (and we may then identify the brane at $y=L$ as the visible brane).

If $q_{1}=-q_{0}$, as considered in [7], then $y_{0}= \pm \infty$, so $H(0)=H(L)=0$ (see Fig. 4) and therefore $\left|q_{0}\right|=2 k$ for any finite non-zero $L$. In this limit,

$$
A(y)=\sinh ^{2}\left[k\left(y-y_{0}\right)\right] / \sinh ^{2}\left[k y_{0}\right] \rightarrow e^{-2 k|y|},
$$

the same as in the RS model [7]. In general, our two brane model is qualitatively different from [7], since it involves two branes with tensions of different magnitudes and not necessarily opposite signs, and interprets the bulk cosmological constant as an integration constant derived from the brane tensions and separation, but the RS model can be obtained as an appropriate limit of the more general model presented here.

Figure 5, in which we plot $e^{-l}$ versus $H^{2}$, illustrates the key features of Eq. (75). The dashed line represents the two brane model described above, and as $l$ increases, the $\Lambda_{\text {eff }}$ follows the dashed line and approaches zero. For the RS model $q_{0}+q_{L}=0=H^{2}$ for any separation $l$. (Of course, $q_{0}$ $+q_{L}=0$ is a fine-tuning.) Thus, the corresponding curve for the RS model is the solid vertical line at $H^{2}=0$. Suppose we start with only one brane, which may be considered as the 


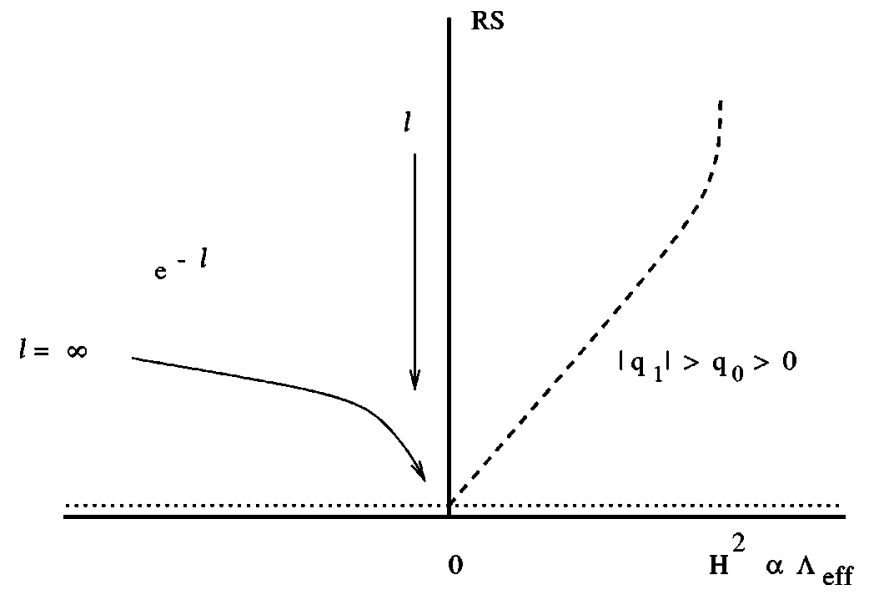

FIG. 5. Hubble constant squared versus separation. More precisely, $e^{-l}=e^{-k L}$ vs $H^{2}$, which is proportional to the $4 \mathrm{D}$ effective cosmological constant $\Lambda_{e f f}$. The dashed line corresponds to our model. The solid vertical line corresponds to the Randall-Sundrum model. The horizontal dotted line corresponds to the single brane model. $l=\infty$ at the intersection of the 3 lines.

$l=\infty$ limit of the two brane model. Then we find that the visible brane (at $y=0$ ) can have any value of $\Lambda_{\text {eff }}$. This happens because the problem has reduced to that of a single brane, i.e., the jump condition for the brane at $y=L=\infty$ is absent, so $k$ is no longer determined. It follows that $H^{2}$, and hence $\Lambda_{e f f}$, can have any value; this is shown by the dotted line along the $x$-axis. This illustrates that the difference is due to the interchange of limits, i.e., the difference between solving the Einstein equation and before taking $l \rightarrow \infty$, versus starting with $l=\infty$ before solving the Einstein equation. For large, finite $l$, we see that one does not have to tune the brane tensions to obtain an exponentially small $\Lambda_{e f f}$, as long as the brane separation is relatively large.

For positive tension branes, there will always be a zero of $A(y)$ between the branes. This "particle horizon," to be discussed below, is a barrier through which communication is impossible in finite time. In light of this, it is more useful to express the Hubble constant on the visible brane not in terms of the tension of the brane at $L$ and its location which can never be detected, but only with $q_{0}$, and $y_{0}$, the detectable quantities. Then Eqs. (75),(76) are

$$
H^{2}(0)=\frac{q_{0}^{2}}{4}\left[1-\tanh ^{2}\left(k y_{0}\right)\right] \simeq \frac{q_{0}^{2}}{2} e^{-q_{0} y_{0}}
$$

where $y_{0}$ is a function of $q_{0}, q_{L}$ and $L$. We discuss the role of horizons in determining the expansion rate on a given brane more completely in Sec. VII C.

For positive tension branes, we shall see that the presence of the interbrane horizons prevents us from explaining the mass hierarchy problem, although we can obtain an exponentially small cosmological constant on one of the two branes-which we can identify as the visible brane-in that case. When one of the branes has negative tension, there are no horizons, and the warp factor will be small on that brane. Hence, we identify the negative tension brane as the visible brane, and can explain the mass hierarchy problem if the warp factor $A(y)$ is sufficiently small on that brane. In that case, we also find that the expansion rate on the visible brane is small, but not small enough to explain the cosmological constant problem: when scaled appropriately, the expansion rate is of order the Higgs boson mass, which is far smaller than the Planck mass, but far larger than the expansion rate of our Universe. To find a solution to both problems, we need more than one separation distance and one bulk integration constant. In general, this may be achieved in a multibrane world, with more than two branes. However, we may also realize such a solution in the two brane case, provided that the brane world is compactified but not orbifolded. The properties of this model are discussed in detail in Sec. V, and from a different perspective.

\section{THE COSMOLOGICAL CONSTANT AND THE HIERARCHY PROBLEMS}

The above two brane orbifold model is able to provide an explanation for either the hierarchy [7] or cosmological constant problem [4], but not both. However, it is possible to solve both problems simultaneously in the multibrane world generally. In this section, we present details of a relatively simple model that solves both problems: a two brane model in which the fifth dimension is compactified, but not orbifolded. To capture the key feature, which is generic in a multibrane world, let us first present a toy model.

\section{A. Probing a one-brane orbifold model}

It is easy to construct a variation of the above two brane $S^{1} / Z_{2}$ orbifold model that foreshadows how the mass hierarchy and cosmological constant problems might be solved simultaneously in a two-brane model. Cut out the line segment $y_{0} \leqslant y \leqslant L$ and remove the $q_{1}$ brane of the above $S^{1} / \mathbb{Z}_{2}$ model, so the resulting orbifold model has length $y_{0}$ (with orbifold fixed points at $y=0$ and $\left.y=y_{0}\right)$. The particle horizon is now at the orbifold fixed point at $y=y_{0}$, and $A\left(y_{0}\right)$ $=0=A^{\prime}\left(y_{0}\right)$ at that point. There is only one brane (the $q_{0}$ brane at the fixed point $y=0$ ) in this $S^{1} / Z_{2}$ orbifold model. $H^{2}$ is still given by Eq. (79) for $q_{0} y_{0} \gg 1$, which is exponentially small. Let us treat the $q_{0}$ brane as the Planck brane, with $A(0)=1$, and introduce the visible brane as a probe brane (i.e., with negligible brane tension, as in Ref. [30]) at $y=L_{v}$, somewhere between the two fixed points. The warp (or hierarchy scale) factor on the visible brane is then $A\left(L_{v}\right) \simeq e^{-q_{0} L_{v}}$, whereas $\Lambda_{e f f} \propto e^{-q_{0} y_{0}}$. Since $y_{0}>L_{v}$, the inequality (4) follows. In this simple two brane model, we see that, if the hierarchy scale factor is exponentially small, the 4D effective cosmological constant must be exponentially smaller. We can of course choose to keep the $q_{1}$ brane for a three brane model.

This model is strictly correct only if the visible brane tension is exactly zero, which is a fine-tuning. A more careful treatment is necessary for an arbitrary visible brane tension, even if it is very small. 


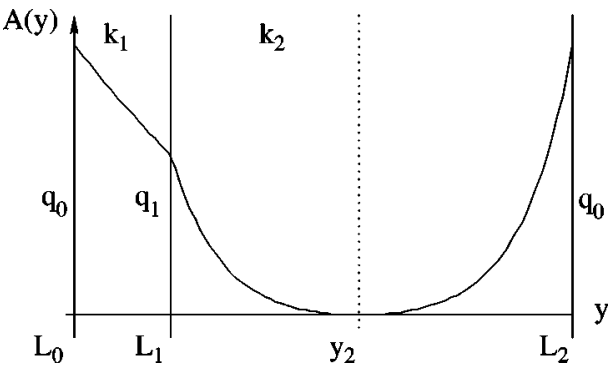

FIG. 6. The two brane compactified model, in which the hierarchy problem and the cosmological constant problem may be simultaneously solved. $L_{0}$ is identified with $L_{2}$, so the circle has circumference $L_{2}-L_{0}$. The brane at $L_{0}\left(L_{1}\right)$ is the Planck (visible) brane. The metric factor $A(y)$ is shown schematically.

\section{B. Two brane compactified model}

Consider a two brane compactified model, which has a $S^{1}$ compactified 5 th dimension, with circumference $L_{2}-L_{0}$, as shown in Fig. 6. The brane at $L_{0}$ has tension $\sigma_{0}$ and the brane at $L_{1}$ has tension $\sigma_{1}$, both of which are positive. Recall that $q_{0}=\kappa^{2} \sigma_{0} / 3$ and $q_{1}=\kappa^{2} \sigma_{1} / 3$. These two branes are separated by $L_{1}-L_{0}$ on one side and $L_{2}-L_{1}$ on the other side of the circle, where $L_{0}$ is identified with $L_{2}$. Without loss of generality, let $\sigma_{0}>\sigma_{1}>0\left(q_{0}>q_{1}>0\right)$. Note that we require two integration constants, namely, the two bulk cosmological constants $\Lambda_{1}$ and $\Lambda_{2}$, or equivalently, $k_{1}$ and $k_{2}$.

For a piecewise constant bulk cosmological constant, we can introduce two 5-form field strengths, or alternatively, start with the model (33) with only one 5-form field strength. Because the 5th dimension is compactified, the brane charges, which are constant parameters, must add to zero: $e_{0}=-e_{1}$. Here $k_{1}^{2}=-\kappa^{2}\left[\phi-\left(e_{(0)}+e_{0}\right)^{2} / 2\right] / 6$ and $k_{2}^{2}=$ $-\kappa^{2}\left(\phi-e_{(0)}^{2} / 2\right) / 6$, where the integration constants are the constant background field strength $e_{(0)}>0$ and the constant $\phi<0$. This allows us to treat $k_{1}$ and $k_{2}$ as integration constants to be determined by the 5D Einstein equation.

The solution $A(y)$ in the bulks is given by Eq. (42) and $H\left(L_{i}\right)$ is given by Eq. (45). The continuity conditions of the metric $A(y)$ at the branes are Eq. (43)

$$
\begin{aligned}
& \frac{\sinh ^{2}\left[k_{1}\left(L_{1}-y_{1}\right)\right]}{k_{1}^{2}}=\frac{\sinh ^{2}\left[k_{2}\left(L_{1}-y_{2}\right)\right]}{k_{2}^{2}} \\
& \frac{\sinh ^{2}\left[k_{2}\left(L_{2}-y_{2}\right)\right]}{k_{2}^{2}}=\frac{\sinh ^{2}\left[k_{1}\left(L_{0}-y_{1}\right)\right]}{k_{1}^{2}}
\end{aligned}
$$

and the jump conditions at the branes are

$$
\begin{aligned}
& q_{0}=k_{2} \operatorname{coth}\left[k_{2}\left(L_{2}-y_{2}\right)\right]-k_{1} \operatorname{coth}\left[k_{1}\left(L_{0}-y_{1}\right)\right] \\
& q_{1}=k_{1} \operatorname{coth}\left[k_{1}\left(L_{1}-y_{1}\right)\right]-k_{2} \operatorname{coth}\left[k_{2}\left(L_{1}-y_{2}\right)\right] .
\end{aligned}
$$

It is straightforward to obtain the large separation behavior. However, let us first get an overall picture of the model. The above conditions can be rewritten as

$$
\begin{aligned}
k_{1}^{2}\left[\operatorname{coth}^{2}\left[k_{1}\left(L_{1}-y_{1}\right)\right]-1\right] & =k_{2}^{2} \frac{\left(\operatorname{coth}^{2}\left[k_{2}\left(L_{2}-y_{2}\right)\right]-1\right)\left(1-t_{2}^{2}\right)}{\left(1-t_{2} \operatorname{coth}\left[k_{2}\left(L_{2}-y_{2}\right)\right]\right)^{2}} \\
k_{2}^{2}\left[\operatorname{coth}^{2}\left[k_{2}\left(L_{2}-y_{2}\right)\right]-1\right] & =k_{1}^{2} \frac{\left(\operatorname{coth}^{2}\left[k_{1}\left(L_{1}-y_{1}\right)\right]-1\right)\left(1-t_{1}^{2}\right)}{\left(1-t_{1} \operatorname{coth}\left[k_{1}\left(L_{1}-y_{1}\right)\right]\right)^{2}} \\
q_{0} & =k_{2} \operatorname{coth}\left[k_{2}\left(L_{2}-y_{2}\right)\right]-k_{1} \frac{\operatorname{coth}\left[k_{1}\left(L_{1}-y_{1}\right)\right]-t_{1}}{1-t_{1} \operatorname{coth}\left[k_{1}\left(L_{1}-y_{1}\right)\right]} \\
q_{1} & =k_{1} \operatorname{coth}\left[k_{1}\left(L_{1}-y_{1}\right)\right]-k_{2} \frac{\operatorname{coth}\left[k_{2}\left(L_{2}-y_{2}\right)\right]-t_{2}}{1-t_{2} \operatorname{coth}\left[k_{2}\left(L_{2}-y_{2}\right)\right]},
\end{aligned}
$$

where $t_{1,2} \equiv \tanh \left[k_{1,2} \Delta l_{1,2}\right]$, where $\Delta l_{1} \equiv k_{1}\left(L_{1}-L_{0}\right)$ and $\Delta l_{2} \equiv k_{2}\left(L_{2}-L_{1}\right)$. Let us consider the allowed regions in the $\left(k_{1}, k_{2}\right)$ plane. The allowed regions in Fig. 7 are those for which the cotanh terms in Eq. (81) have the correct signs to correspond to the behavior of the scale factor $A(y)$ in the bulk between the branes. Some of the details appear in Appendix B. Figure 7 shows the physically distinct allowed regions, where one demands $L_{2}-L_{1} \geqslant L_{1}-L_{0}$ without loss of generality. We see that there are two allowed regions:

(1) The "one horizon" region for $k_{2}>k_{1}$ is bounded by the $k_{1}=0$ line, the $q_{0}=k_{1}+k_{2}$ line and the $L_{1}-L_{0}=0$ curve. In this region,

$$
k_{2}\left(L_{2}-L_{1}\right)>k_{1}\left(L_{1}-L_{0}\right)
$$

that is, the particle horizon is between $L_{1}$ and $L_{2}$ segment of the circle, whose bulk cosmological constant has a larger magnitude $\left(k_{2}>k_{1}\right)$. (The other $k_{1} \geqslant k_{2}>0$ region has an identical description, and they are related by a simple interchange of the two segments of the circle.)

(2) The "two horizon" region, where there is a particle horizon in each of the two segments of the circle. 


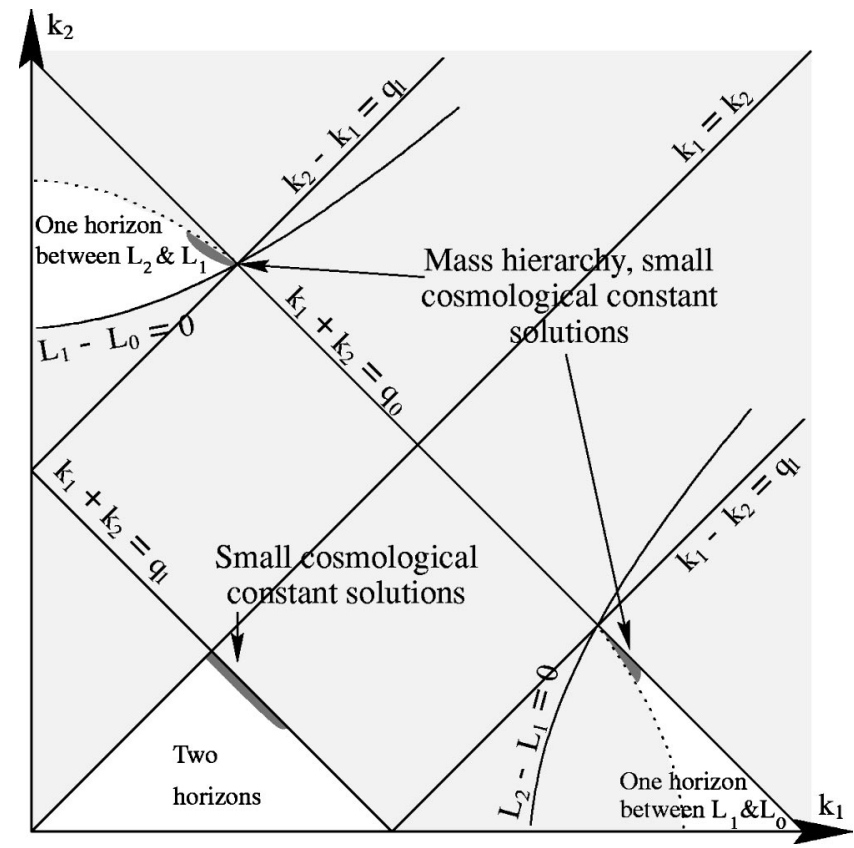

FIG. 7. The allowed regions (white) of " $k$-space" for which there are consistent solutions with $H^{2}\left(L_{1,2}\right)>0$ and $L_{2}-L_{1}>L_{1}$ $-L_{0}$. The regions with small cosmological constant solutions are shaded dark.

Let us consider the "one horizon" region where $k_{2} \geqslant k_{1}$ $>0$. This region is bounded by the $k_{1}=0$ line, the $q_{0}=k_{1}$ $+k_{2}$ line and the $L_{1}-L_{0}=0$ curve. The $L_{1}-L_{0}=0$ curve is given by the equation $k_{2}^{2}=k_{1}^{2}+q_{0} q_{1}$, which can be derived from Eq. (83). In this region, $k_{2}\left(L_{2}-L_{1}\right)>k_{1}\left(L_{1}-L_{0}\right)$, that is, the particle horizon is between the $L_{1}$ and $L_{2}$ segment of the circle, that is, the segment with a larger magnitude bulk cosmological constant $\left(k_{2}>k_{1}\right)$. This region is further divided into two subregions, where $\left(L_{2}-L_{1}\right)<\left(L_{1}-L_{0}\right)$ (the upper part) and $\left(L_{2}-L_{1}\right) \geqslant\left(L_{1}-L_{0}\right)$ (the lower part). Because of the relationship between $\Delta l_{i}$ and the behavior of the scale factors in the bulk, for Fig. 6 we have

$$
\begin{aligned}
& \Delta l_{1} \equiv k_{1}\left(L_{1}-L_{0}\right)=\sinh ^{-1}\left(\frac{k_{1}}{H\left(L_{0}\right)}\right)-\sinh ^{-1}\left(\frac{k_{1}}{H\left(L_{1}\right)}\right) \\
& \Delta l_{2} \equiv k_{2}\left(L_{2}-L_{1}\right)=\sinh ^{-1}\left(\frac{k_{2}}{H\left(L_{1}\right)}\right)+\sinh ^{-1}\left(\frac{k_{2}}{H\left(L_{2}\right)}\right) .
\end{aligned}
$$

\section{Large brane separations}

It is easy to see that as $H\left(L_{1}\right) \rightarrow 0, \Delta l_{2} \rightarrow \infty$, but $\Delta l_{1}$ can remain positive and relatively small if $H\left(L_{0}\right) \equiv H\left(L_{2}\right)$ is smaller than $H\left(L_{1}\right)$. The brane at $L_{1}$ will then be identified as the visible brane, on which $A\left(L_{1}\right)$ is large compared to $H\left(L_{1}\right)$ there, yet still exponentially small when compared to the fundamental (Planck) scale.

Following the remarks above, we expect $\Delta l_{2}$ to be large $\left(t_{2} \rightarrow 1\right)$. The boundary condition at $L_{1}$ can then be satisfied if $k_{1} \rightarrow 0$, or $\operatorname{coth}\left[k_{1}\left(L_{1}-y_{1}\right)\right] \rightarrow-1$. The later solution will permit the two different scales required. The second boundary condition will require $\operatorname{coth}\left[k_{2}\left(L_{2}-y_{2}\right)\right] \rightarrow 1$. Writing

$$
\begin{aligned}
t_{2} & \simeq 1-2 \exp \left[-2 \Delta l_{2}\right] \\
\operatorname{coth}\left[k_{1}\left(L_{1}-y_{1}\right)\right] & \simeq-1-\eta_{1} \\
\operatorname{coth}\left[k_{2}\left(L_{2}-y_{2}\right)\right] & \simeq 1+\eta_{2},
\end{aligned}
$$

where $\eta_{1,2}$ are expected to be $O\left(\exp \left[-\Delta l_{2}\right]\right)$. To zeroth order in $\eta_{1,2}$, the jump conditions are satisfied if

$$
k_{1}=\frac{1}{2}\left(q_{0}-q_{1}\right), \quad k_{2}=\frac{1}{2}\left(q_{0}+q_{1}\right)
$$

We imposed $q_{0}>q_{1}$ which give $k_{i}>0$ as required for consistency. The boundary conditions to first order in $\eta_{1,2}$ lead to

$$
\begin{aligned}
& \eta_{1}=2\left(\frac{q_{0}+q_{1}}{q_{0}-q_{1}}\right)^{2} \sqrt{\left(\frac{1+t_{1}}{1-t_{1}}\right)} e^{-\Delta l_{2},} \\
& \eta_{2}=2 \sqrt{\left(\frac{1-t_{1}}{1+t_{1}}\right)} e^{-\Delta l_{2}} .
\end{aligned}
$$

We now demand that $\Delta l_{1}$ is large, but it must remain smaller than $\Delta l_{2}$ (see Appendix B) so $t_{1} \simeq 1-2 \exp \left[-2 \Delta l_{1}\right]$, which gives

$$
\begin{aligned}
& H^{2}\left(L_{0}\right)=H^{2}\left(L_{2}\right) \simeq\left(q_{0}+q_{1}\right)^{2} e^{-\left(\Delta l_{2}+\Delta l_{1}\right)}, \\
& H^{2}\left(L_{1}\right) \simeq\left(q_{0}+q_{1}\right)^{2} e^{-\left(\Delta l_{2}-\Delta l_{1}\right)} .
\end{aligned}
$$

Figure 6 shows schematically that the scale factor on the $L_{1}$ brane is exponentially smaller than on the $L_{0}$ brane:

$$
\frac{A\left(L_{1}\right)}{A\left(L_{0}\right)}=\frac{H^{2}\left(L_{0}\right)}{H^{2}\left(L_{1}\right)} \simeq e^{-2 \Delta l_{1}}
$$

to lowest order. For a compactified 5th dimension, with both $\Delta l_{1} \simeq\left(q_{0}-q_{1}\right)\left(L_{1}-L_{0}\right) / 2$ and $\Delta l_{2} \simeq\left(q_{0}+q_{1}\right)\left(L_{2}-L_{1}\right) / 2$ large, we find that $A\left(L_{1}\right)$ and $H^{2}\left(L_{0}\right)$ and $H^{2}\left(L_{1}\right)$ are all exponentially small. The results are

$$
\begin{aligned}
A\left(L_{1}\right) & \simeq e^{-\left(q_{0}-q_{1}\right)\left(L_{1}-L_{0}\right)} \\
H^{2}\left(L_{0}\right) & \simeq\left(q_{0}+q_{1}\right)^{2} e^{-\left[\left(q_{0}+q_{1}\right)\left(L_{2}-L_{1}\right)+\left(q_{0}-q_{1}\right)\left(L_{1}-L_{0}\right)\right] / 2} \\
H^{2}\left(L_{1}\right) & \simeq\left(q_{0}+q_{1}\right)^{2} e^{-\left[\left(q_{0}+q_{1}\right)\left(L_{2}-L_{1}\right)-\left(q_{0}-q_{1}\right)\left(L_{1}-L_{0}\right)\right] / 2} .
\end{aligned}
$$


Since $L_{1}>L_{0}$ and $q_{0}>q_{1}$, the warp factor $A\left(L_{1}\right)$ is exponentially small.

\section{D. $G_{N}$ and $\Lambda_{e f f}$}

Let us consider further the situation as shown in Fig. 6 in terms of the $4 \mathrm{D}$ effective action. The $y=L_{0}=0$ brane is referred to as the Planck (hidden) brane and the $y=L_{1}$ brane is referred to as the $\mathrm{TeV}$ (visible) brane. (Note that the visible brane tension may be of order Planck scale.) We adopt $A(0) \equiv 1$, so $A\left(L_{1}\right)=e^{-2 \Delta l_{1}}$, where $\Delta l_{1} \simeq\left(q_{0}-q_{1}\right) L_{1} / 2$. We shall take $A\left(L_{1}\right) \simeq 10^{-32}$, so the mass $m_{H 1}=\sqrt{A\left(L_{1}\right)} M_{H 1}$ of the Higgs field $\hat{\phi}_{1}$ on the visible brane is around $\mathrm{TeV}$, while the mass $m_{H 0}=\sqrt{A(0)} M_{H 0}$ of the Higgs field $\hat{\phi}_{0}=\phi_{0}$ on the hidden brane is comparable to the Planck mass. In this model, the $y$ integration is over the entire $S^{1}$. Up to exponentially small corrections, we have

$$
\int A(y) d y=\int \frac{H^{2}}{k^{2}} \sinh ^{2}\left[k\left(y-y_{0}\right)\right] d y \simeq \frac{1}{2 k_{1}}+\frac{1}{2 k_{2}}
$$

with $k=k_{1}, k_{2}$ for each side of the $y=L_{0}$ brane, so, up to exponentially small corrections, this integral over $y$ yields the same $G_{N}$ as that in Eq. (57). In terms of brane tensions,

$$
\frac{1}{\kappa_{N}^{2}} \simeq \frac{2 q_{0}}{\left(q_{0}^{2}-q_{1}^{2}\right) \kappa^{2}}
$$

Using the result Eq. (88) for $H^{2}$, we find that the effective $4 \mathrm{D}$ cosmological constant is

$$
\Lambda_{e f f} \simeq \frac{2 \sigma_{0}\left(\sigma_{0}+\sigma_{1}\right)}{\left(\sigma_{0}-\sigma_{1}\right)} e^{-\Delta l_{2}-\Delta l_{1}}
$$

where $\Delta l_{1} \simeq\left(q_{0}-q_{1}\right)\left(L_{1}-L_{0}\right) / 2$ and $\Delta l_{2} \simeq\left(q_{0}+q_{1}\right)\left(L_{2}\right.$ $\left.-L_{1}\right) / 2$. In terms of $\hat{\phi}_{i}$ and $m_{H i}, G_{N}$ and $\Lambda_{\text {eff }}$ are the same for observers on both the visible (i.e., the TeV) brane at $y$ $=L_{1}$ and on the Planck brane at $y=L_{0}=0$. Since $H^{2}$ $=\kappa_{N}^{2} \Lambda_{e f f} / 3$, we expect the same Hubble constant for the two branes. However, Eq. (86) gives the ratio $H^{2}\left(L_{0}\right) / H^{2}\left(L_{1}\right)$ $=A\left(L_{1}\right)$. This difference arises because Eq. (86) is calculated in the $\left(\gamma_{\mu \nu}, \phi_{i}, M_{H i}\right)$ frame, while properties calculated from the effective action $\mathbf{S}^{(4)}$ are in the $\left(\hat{\gamma}_{\mu \nu}, \hat{\phi}_{i}, m_{H i}\right)$ frame. That is, in the $\left(\hat{\gamma}_{\mu \nu}, \hat{\phi}_{i}, m_{H i}\right)$ frame, the Hubble constant seen by observers on the visible brane is simply $H^{2}$ $=H^{2}\left(L_{0}\right)$. [More details on such $A\left(L_{i}\right)$ rescaling later.]

As pointed out in Ref. [7], the Higgs boson mass $m_{\text {Higgs }}$ as seen by observers on the visible brane is given by $m_{\text {Higgs }}^{2}=A\left(L_{1}\right) M_{H}^{2}$. Using Eqs. (85),(88),(125), we see that the observed Higgs boson mass is exponentially small compared to the Planck mass for large brane separation $L_{1}=L_{1}$ $-L_{0}>0$,

$$
\begin{aligned}
\frac{m_{\text {Higg }}^{2}}{m_{\text {Planck }}^{2}} & \simeq \frac{2 k_{1} k_{2} M_{H}^{2} A\left(L_{1}\right)}{\left(k_{1}+k_{2}\right) M^{3}} \\
& =\frac{4 \pi M_{H}^{2}\left(\sigma_{0}^{2}-\sigma_{1}^{2}\right)}{3 M^{6} \sigma_{0}} e^{-8 \pi\left(\sigma_{0}-\sigma_{1}\right) L_{1} / 3 M^{3}}
\end{aligned}
$$

for $\sigma_{0}>\sigma_{1}>0$. Thus, we obtain a Randall-Sundrum like explanation of the hierarchy problem: an appropriate choice of the the warp factor can explain the hierarchy problem. At the same time $\hat{\phi}_{1}^{6}$ and other higher-dimensional operators become more important in the effective theory as their couplings are exponentially enhanced, that is, $m_{c 1}$ becomes $\mathrm{TeV}$ scale.

\section{E. An inequality}

It is clear from the above that the mass hierarchy problem can be explained by this model if $A\left(L_{1}\right) \sim 10^{-32}$. What is not so obvious, but shall be explained in Sec. VIII, is that the appropriate statement of the cosmological constant problem is that $H^{2}\left(L_{0}\right) G_{N} \sim 10^{-122}$. This may be understood most easily in terms of the effective theory introduced in Sec. III B, where we show that, with appropriate choice of units, $G_{N}$ and $H$ may be regarded as the same on all branes, but the Higgs boson mass on any particular brane is related to $G_{N}$ by $G_{N} m_{\text {Higgs }}^{2} \sim A\left(L_{i}\right)$. Assuming that $q_{0}, q_{1} \sim G_{N}^{-1 / 2}$, we see that Eq. (88) [as well Eqs. (87) and (86)] implies that if we can explain the mass hierarchy problem, then the observed expansion rate, in Planck units, is smaller than the observed Higgs boson mass in Planck units by additional powers of $A\left(L_{i}\right) \ll 1$, because $\Delta l_{2}>\Delta l_{1}$. Although the static models do not fix the size of expansion rate precisely, even when the ratio of Higgs boson and Planck masses is taken from observation, they guarantee that the value is far smaller than the Higgs boson mass. These considerations are based on the one-horizon model, and the inequality $\Delta l_{2}>\Delta l_{1}$.

In the above analysis, we show how two exponentially small factors can be generated, one for the hierarchy problem and the other for the cosmological constant problem. As an input, we choose a large radius $\left(L_{2}-L_{0}\right) / 2 \pi$ for the compactification. It is important to find a dynamical reason for such a large radius. Here let us address a much more modest question: why should the exponent for the effective cosmological constant, $m_{\text {Planck }}^{4} / \Lambda_{\text {eff }}$, be bigger than the exponent for the hierarchy factor, $m_{\text {Planck }}^{2} / m_{\text {Higg }}^{2}$ ? Since both exponential factors have similar origins, we actually have an inequality between these two exponents that is satisfied in nature.

The solution we have considered is the "one horizon" region in Fig. 7. As we approach $q_{0}=k_{1}+k_{2}$ and $q_{1}=k_{2}$ $-k_{1}$, both $L_{1}-L_{0}$ and $L_{2}-L_{1}$ become large. Since $\Delta l_{2}$ $>\Delta l_{1}$ [that is, $\left.\left(q_{0}+q_{1}\right)\left(L_{2}-L_{1}\right)>\left(q_{0}-q_{1}\right)\left(L_{1}-L_{0}\right)\right]$, we have the inequality (4), namely, $\ln \left(m_{\text {Planck }}^{4} / \Lambda_{\text {eff }}\right)$ $>\ln \left(m_{\text {Planck }}^{2} / m_{\text {Higgs }}^{2}\right)$, as is the case in nature. However, we can actually make a stronger inequality, that is, $L_{2}-L_{1}>L_{1}$ $-L_{0}$ with $q_{0}+q_{1}>q_{0}-q_{1}$ (i.e., the particle horizon is in the 
larger segment of the circle). Let us assume that the positions of the branes are fixed, that is, the stationary branes satisfy their corresponding equations of motion. There are two distinct cases here:

(a) $L_{2}-L_{1}>L_{1}-L_{0}>0$ and $k_{2}>k_{1}$; that is, the particle horizon is in the larger segment of the circle between the branes. This case is schematically shown in Fig. 6.

(2) The particle horizon is in the shorter segment of the circle. There are two equivalent descriptions of this case. We can either fix $L_{2}-L_{1}>L_{1}-L_{0}>0$, which implies that $\Delta l_{2}$ $<\Delta l_{1}$ and $k_{1}>k_{2}$; or choose $L_{1}-L_{0}>L_{2}-L_{1}>0$ in the $k_{2}$ $>k_{1}$ region with $\Delta l_{2}>\Delta l_{1}$. The Hubble constant in this sec- ond case (with the particle horizon in the shorter segment of the circle) is given by

$$
\widetilde{H}^{2}\left(L_{0}\right)=\left(q_{0}+q_{1}\right)^{2} e^{-\left(\widetilde{\Delta} l_{2}+\widetilde{\Delta} l_{1}\right)}
$$

where $\widetilde{\Delta} l_{2}=\left(q_{0}-q_{1}\right)\left(L_{2}-L_{1}\right) / 2$ and $\widetilde{\Delta} l_{1}=\left(q_{0}+q_{1}\right)\left(L_{1}\right.$ $\left.-L_{0}\right) / 2$.

Nature will pick the lower energy (or least action) solution, that is, the one with smaller $\Lambda_{\text {eff }}$. Since the Newton's constant is essentially the same in both cases (more discussion on this later), we can simply compare this Hubble constant to that in Eq. (86):

$$
\begin{gathered}
\left(q_{0}+q_{1}\right)\left(L_{2}-L_{1}\right)+\left(q_{0}-q_{1}\right)\left(L_{1}-L_{0}\right)>\left(q_{0}-q_{1}\right)\left(L_{2}-L_{1}\right)+\left(q_{0}+q_{1}\right)\left(L_{1}-L_{0}\right) \\
\Rightarrow L_{2}-L_{1}>L_{1}-L_{0} .
\end{gathered}
$$

This implies that the particle horizon should be in the larger segment between the branes, as schematically shown in Fig. 6 , where $L_{2}-L_{1}>L_{1}-L_{0}>0$ and $k_{2}>k_{1}$. Note that this inequality $L_{2}-L_{1}>L_{1}-L_{0}$ together with $q_{0}>q_{1}>0$ is stronger than the inequality $\left(q_{0}+q_{1}\right)\left(L_{2}-L_{1}\right)>\left(q_{0}-q_{1}\right)\left(L_{1}\right.$ $\left.-L_{0}\right)$. Using this inequality (94) and $q_{0}>q_{1}>0$, we can now compare the exponent in Eq. (88), where

$$
\frac{m_{\text {Higgs }}^{2}}{m_{\text {Planck }}^{2}} \simeq A\left(L_{1}\right) \simeq e^{-\left(q_{0}-q_{1}\right)\left(L_{1}-L_{0}\right)}
$$

and the exponent in Eq. (86), where we can write, in terms of $\Lambda_{e f f}$,

$$
\Lambda_{e f f} \simeq \frac{2 \sigma_{0}\left(q_{0}+q_{1}\right)}{\left(q_{0}-q_{1}\right)} e^{\left[\left(q_{0}+q_{1}\right)\left(L_{2}-L_{1}\right)+\left(q_{0}-q_{1}\right)\left(L_{1}-L_{0}\right)\right] / 2}
$$

to obtain

$$
\ln \left(\frac{m_{\text {Planck }}^{4}}{\Lambda_{\text {eff }}}\right)>\ln \left(\frac{m_{\text {Planck }}^{2}}{m_{\text {Higgs }}^{2}}\right)
$$

as is the case in nature, where $\ln \left(m_{\text {Planck }}^{4} / \Lambda_{\text {eff }}\right) \sim 2.3 \times 122$ and $\ln \left(m_{\text {Planck }}^{2} / m_{\text {Higgs }}^{2}\right) \sim 2.3 \times 32$. To get a feeling for the magnitudes of the various quantities, we see that choosing $L_{2}$ $-L_{1} \sim 2\left(L_{1}-L_{0}\right)$ and $\sigma_{0} \sim 2 \sigma_{1}$ gives the correct order of magnitude. If we assume that the brane tensions are of Planck scale, the values of the cosmological constant and mass hierarchy observed in nature follow if $\left(L_{1}-L_{0}\right)$ $\sim 10 m_{\text {Planck. }}^{-1}$. Smaller brane tensions imply larger $\left(L_{1}-L_{0}\right)$.

In this model, the branes are stationary (that is, they satisfy the brane equations of motion) and both $L_{1}$ and $L_{2}$ are stable. But we still need a dynamical explanation for why $L_{1}$ (or $L_{2}$ ) is large. However, if we understand the hierarchy problem via some means, then this brane world model provides an explanation of the cosmological constant problem.
Now, the hierarchy problem may be understood from the renormalization group flow in $4 \mathrm{D}$ quantum field theory. It is well known that the running of the couplings (marginal operators) are logarithmic versus the running of the mass terms (relevant operators). A well-known example is the unification of the couplings in some supersymmetric versions of the standard model. If we accept some version of this picture as a resolution of the hierarchy problem, then the cosmological constant problem is explained by the inequality (4) that appears in this two brane world model. In fact, the running of the couplings and masses in 4D quantum field theory can be recast as the holographic renormalization group flow in the brane world scenario [31]. So, in a brane world model where the hierarchy problem is solved, the inequality (4) tells us that we have an exponentially small 4D cosmological constant.

\section{F. Two-horizon region}

There is another possible solution, namely, the "two horizon" region shown in Fig. 7. In this case, the two branes are separated by particle horizons. Since we are approaching $q_{1}=k_{2}+k_{1}$, the value of $H^{2} / A(0)$ is finite, so the $q_{1}$ brane again has exponentially small cosmological constant. Within the 5D classical Einstein theory, the two solutions are topologically distinct and so we cannot compare them. However, one may imagine a deviation from the pure AdS situation, or in quantum gravity or string or M theory, where they may be compared. Which solution does the two brane system pick? As is discussed in more detail in Appendix B, the 4D effective theory has a well-defined $\Lambda_{e f f}$, so we may compare the values of $\Lambda_{e f f}$ in the two cases. For large brane separation $L_{2}-L_{1}$, we have seen that $\Lambda_{\text {eff }}$ is exponentially small in both cases. By comparing $\Lambda_{e f f}$ in the two cases, the "one horizon" case (where the hierarchy problem may be solved) has a smaller $\Lambda_{e f f}$, due to smaller $k_{2}$ and maybe also $k_{1}$. For finite brane separations, and with appropriate choices of $q_{i}$, it is possible that the second case is preferred. One can imag- 
ine that the brane world starts with (two) particle horizons separating the two branes. As the brane separations (and the radius of $S^{1}$ ) increase, the bulk cosmological constants on two sides jump to the values for the hierarchy solution phase. We do not expect this to happen in the 5D theory. However, within the string or M theory framework, such a transition is akin to a topological change, a possibility that deserves further study.

\section{MULTIBRANE WORLD}

It should be clear that a three brane or multibrane model can provide a similar solution to both fine tuning problems by the same mechanism; on the visible brane $H^{2}$ $\sim \exp \left(-2 \Delta l_{2}\right)$, with a coefficient dependent on all three brane tensions, and $A\left(L_{1}\right) / A\left(L_{0}\right) \simeq \exp \left(-2 \Delta l_{1}\right)$. However, the 5-form field strength mechanism to provide piecewise constant bulk energy densities fails in an orbifolded space and it is not currently known how to achieve the different adjustable bulk cosmological constants. A multibrane world with 5 -form field strengths providing the piecewise bulk cosmological constant can give a solution to both fine-tuning problems, in non-orbifolded spaces. Other variations of the models studied here should be explored further.

We can now give a general picture of the physics when the brane separations are large. In the stationary situation, we shall treat the brane positions $L_{i}$ as fixed, while the parameters $k_{i}$ between branes and $y_{i}$ are to be determined by the jump conditions, via Eqs. (43) and (44). By solving the jump conditions, the Hubble constant seen by observers on the $i$ th brane can be expressed in terms of the 5D gravitational coupling $\kappa$, its brane tension $\sigma_{i}$ and the tensions $\sigma_{i-1}$ and $\sigma_{i+1}$ and positions $L_{i-1}$ and $L_{i+1}$ of its neighboring branes. For large brane separations the relation (44) between the bulk parameters $k_{i}$ and $k_{i+1}$ and the brane tension becomes

$$
\begin{aligned}
q_{i} & =k_{i} \operatorname{coth}\left[k_{i}\left(L_{i}-y_{i}\right)\right]-k_{i+1} \operatorname{coth}\left[k_{i+1}\left(L_{i}-y_{i+1}\right)\right], \\
& \rightarrow s_{i}^{-} k_{i}-s_{i}^{+} k_{i+1}
\end{aligned}
$$

where $s_{i}^{+}$is the sign of $A^{\prime}$ at $y \rightarrow L_{i}^{+}$, and the corrections are exponentially small for large separations. Therefore, for any of the four possible sign choices we see that $H^{2}\left(L_{i}\right)$ becomes exponentially small. The implications of the various sign choices for the cosmological constant and hierarchy problems can be summarized qualitatively in Table I, where it is assumed that $k_{i+1} \geqslant k_{i}>0$. (The opposite inequality $k_{i+1}$ $\leqslant k_{i}$ does not yield any new possibilities, as they can be obtained from Table I by exchanging some of the cases.)

We consider cases for which $H^{2}\left(L_{i}\right)>0$ only. For case (1) the scale factor is locally peaked at the brane. The brane in case (3) can have no zeros between it and either neighboring brane, while the remaining cases may have a horizon on one side only. The $q_{i}$ column gives the sign of the brane tension; stability is expected for a brane with a positive tension. Generically, a negative tension brane is unstable, as in case (4), but, it is possible that some stabilization mechanism may be found in a more complicated model. Stability is assumed for a negative tension brane if it sits at an orbifold fixed point, so
TABLE I. Implications of various sign choices for cosmological constant and hierarchy problems.

\begin{tabular}{|l|l|l|c|c|c|c|c|}
\hline $\mathrm{A}(\mathrm{y})$ & Case & $q_{i}$ & stability & $H^{2}\left(L_{i}\right)$ & $G_{N}$ & Hierarchy \\
\hline \hline 1 & $\mathcal{N}$ & $q_{i} \geq k_{i+1}+k_{i}$ & + & $\sqrt{ }$ & + & + & $\times$ \\
\hline 2 & $\mathcal{Y}$ & $q_{i} \leq k_{i+1}-k_{i}$ & + & $\sqrt{ }$ & + & + & $\sqrt{ }$ \\
\hline 3 & $\bigvee$ & $q_{i} \leq-\left(k_{i+1}+k_{i}\right)$ & - & $?$ & + & + & $\sqrt{ }$ \\
\hline 4 & $\mathcal{Y}$ & $q_{i} \geq-\left(k_{i+1}-k_{i}\right)$ & - & $\times$ & + & + & $\sqrt{ }$ \\
\hline
\end{tabular}

that the fluctuation modes that will cause destabilization have presumably been projected out. In this case, the $\mathbb{Z}_{2}$ symmetry requires $k_{i+1}=k_{i}$. This is represented by "?" in case (3) in Table I.

As we shall explain later, the Newton constant is always positive and is the same on all branes. The value of the 4D Newton's constant $G_{N}$ may be determined by the introduction of a small matter density in case (1), where $A(y)$ is peaked at the brane, as given in Eq. (49). However, the same method cannot be applied to branes in the other cases, where $A(y)$ is not peaked at the brane (see Appendix D).

In the above framework, and without fine-tuning, we see that there can be models that simultaneously

(1) have a stable positive tension brane,

(2) have an exponentially small 4D positive cosmological constant, and

(3) yield a solution to the hierarchy problem.

Self-consistent models with all of these desirable properties require a combination of case (1) and case (2), so the simplest model must contain at least two branes. Generically, a multibrane model can accommodate both an exponentially small cosmological constant and a solution to the hierarchy problem. The model of Ref. [4] corresponds to the case (1) in Table I, in which the hierarchy problem is not solved. We shall show that the hierarchy problem in this model is simply the standard one, so it may be solved by more conventional means. The Randall-Sundrum solution to the hierarchy problem corresponds to the converging limit of case (3), where $k_{i+1}=k_{i}$, with a negative brane tension. The hierarchy problem may be solved when the metric is non-vanishing before it reaches the next brane. This is indicated by a " $\checkmark$ " in case (2) in Table I. The simplest realization of such a solution to both the cosmological constant problem and the hierarchy problem is a two brane model in the compactified (but not orbifold) case. In this model, the hidden Planck brane corresponds to case (1) and the visible brane corresponds to case (2).

In fact, the two brane compactified model, although simplest, is rather specific, and we can generalize the main result to prove that $H^{2} / m_{\text {Higgs }}^{2} \ll 1$ in a multibrane model. Suppose that the visible brane is the $i$ th brane, with a warp factor $A\left(L_{i}\right)$ relative to the value on the Planck brane, which is somewhere to its left, with no particle horizons in the intervening region. Let $D_{i}=L_{i}-L_{i-1}$ be the distance to brane $i$ -1 just to the left of the $i$ th brane, where the warp factor is 
$A\left(L_{i-1}\right)>A\left(L_{i}\right)$, and let $D_{i+1}=L_{i+1}-L_{i}$ be the distance to the brane $i+1$ just to the right of the visible brane, where the warp factor is $A\left(L_{i+1}\right)<A\left(L_{i}\right)$. [This presumes that there is no particle horizon between branes $i$ and $i+1$; if there is, then take $A\left(L_{i+1}\right)=0$ and let $D_{i+1}$ be the distance from the $i$ th brane to the horizon.]

Then instead of Eq. (88), we find

$$
\begin{aligned}
H^{2} \approx & 4 q_{i}^{2} A\left(L_{i}\right)\left[\frac{A\left(L_{i}\right)}{A\left(L_{i-1}\right)}\right]^{D_{i+1} / D_{i}} \\
& \times e^{-2 q_{i} D_{i+1}\left[1+\frac{\ln \left(A\left(L_{i-1}\right) / A\left(L_{i}\right)\right)}{2 q_{i} D_{i}}\right]^{2}} \\
& \times\left[1-\frac{\left[A\left(L_{i+1}\right)\right]^{1 / 2}\left[A\left(L_{i-1}\right)\right]^{D_{i+1} / 2 D_{i}}}{\left[A\left(L_{i}\right)\right]^{1 / 2+D_{i+1} / 2 D_{i}}} e^{q_{i} D_{i+1}}\right] ;
\end{aligned}
$$

once again, we see that $H^{2} / m_{\text {Higgs }}^{2}$ is exponentially small, although the model is more complicated than before. (Consistency requires non-negative $H^{2}$.) The two brane, uncompactified version of Eq. (99), which is appropriate when horizons surround two branes, one of which is the visible brane (with tension $q_{V}$ and warp factor $A_{V}$ ) and the other the Planck brane (with tension $q_{P}$ and warp factor $A_{P}=1$ ) is

$$
H^{2} \approx 4 q_{V}^{2} A_{V}^{1+D_{0} / D_{P}} e^{-2 q_{V} D_{0}}\left[1+\frac{\ln \left(A_{V}^{-1}\right)}{2 q_{V} D_{P}}\right]^{2},
$$

where $D_{0}$ is the distance from the visible brane to the horizon to its right, and $D_{P}$ is the distance from the visible brane to the Planck brane to its left. Equation (88) can be obtained from the uncompactified model by assuming a periodic sequence of identical two brane regions bounded by horizons.

\section{GLOBAL STRUCTURE OF THE SOLUTIONS}

All of the solutions discussed in this paper consist of regions of 5D anti-de Sitter space joined across the 3-branes, together with some additional identifications in some of the solutions. However, the form (36) of the 5D anti-de Sitter metric used here is unconventional, and in addition in some cases possess coordinate singularities where the metric coefficient $A(y)$ vanishes. Coordinate singularities of this type are present in all solutions for which all the branes have positive tension. ${ }^{5}$ As discussed in the Introduction, these zeros of $A(y)$ are particle horizons, in the sense that signals traveling from a brane to the horizon are perceived by observers on that brane to take an infinite time to reach the horizon (although the elapsed proper time or elapsed affine parameter for null signals is finite). These horizons are closely analogous to the horizons in Rindler space. In this section, we show that the coordinate singularities are remov-

\footnotetext{
${ }^{5}$ In the two brane case, the coordinate singularities can be avoided (by choosing the free parameters appropriately) when the two branes are not identical. However, in the symmetric two brane case, a coordinate singularity between the two branes is unavoidable.
}

able by exhibiting the coordinate transformation between the form (36) of the metric and a more standard form of 5D AdS space. We also derive the global structure of the various bulk solutions we have been discussing. The key result that we shall obtain is that the solutions typically split into different disconnected components across the particle horizons. The global structure of the RS model has been discussed in Ref. [32].

\section{A. Different coordinate systems on 5D anti-de Sitter space}

We start by discussing the global structure of the spacetime (36) when no branes are present, so that $L_{i}=-\infty$ and $L_{i+1}=+\infty$. The metric (36) can be written

$$
d s^{2}=d y^{2}-A(y) d t^{2}+A(y) e^{2 H t}\left[\left(d x^{1}\right)^{2}+\left(d x^{2}\right)^{2}+\left(d x^{3}\right)^{2}\right],
$$

where the coefficient $A(y)$ is given by Eq. (42),

$$
A(y)=\frac{H^{2}}{k_{i}^{2}} \sinh ^{2}\left[k_{i}\left(y-y_{i}\right)\right] .
$$

We define new coordinate $T, Y$ and $X^{l}$ for $1 \leqslant l \leqslant 3$ by

$$
\begin{aligned}
T & =\frac{1}{2} \tanh \left[k_{i}\left(y-y_{i}\right) / 2\right]\left\{\left(1+H^{2} \mathbf{x}^{2}\right) e^{H t}-e^{-H t}\right\} \\
Y & =\frac{1}{2} \tanh \left[k_{i}\left(y-y_{i}\right) / 2\right]\left\{\left(1-H^{2} \mathbf{x}^{2}\right) e^{H t}+e^{-H t}\right\} \\
X^{l} & =H \tanh \left[k_{i}\left(y-y_{i}\right) / 2\right] e^{H t} x^{l},
\end{aligned}
$$

where $\mathbf{x}^{2}=\left(x^{1}\right)^{2}+\left(x^{2}\right)^{2}+\left(x^{3}\right)^{2}$. The inverse of this transformation is given by

$$
\begin{aligned}
\tanh ^{2}\left[k_{i}\left(y-y_{i}\right) / 2\right] & =\mathbf{X}^{2}+Y^{2}-T^{2} \\
\exp [2 H t] & =\frac{(T+Y)^{2}}{\mathbf{X}^{2}+Y^{2}-T^{2}} \\
H x^{l} & =\frac{X^{l}}{T+Y},
\end{aligned}
$$

where the sign of $y-y_{i}$ is taken to be the same as the sign of $T+Y$, and where $\mathbf{X}^{2}=\left(X^{1}\right)^{2}+\left(X^{2}\right)^{2}+\left(X^{3}\right)^{2}$. In the new coordinates $\left(T, Y, X^{1}, X^{2}, X^{3}\right)$ the metric takes the standard, conformally-flat AdS form

$$
\begin{aligned}
d s^{2}= & \frac{4 / k_{i}^{2}}{\left(1+T^{2}-\mathbf{X}^{2}-Y^{2}\right)^{2}}\left[-d T^{2}+d Y^{2}+\left(d X^{1}\right)^{2}\right. \\
& \left.+\left(d X^{2}\right)^{2}+\left(d X^{3}\right)^{2}\right] .
\end{aligned}
$$




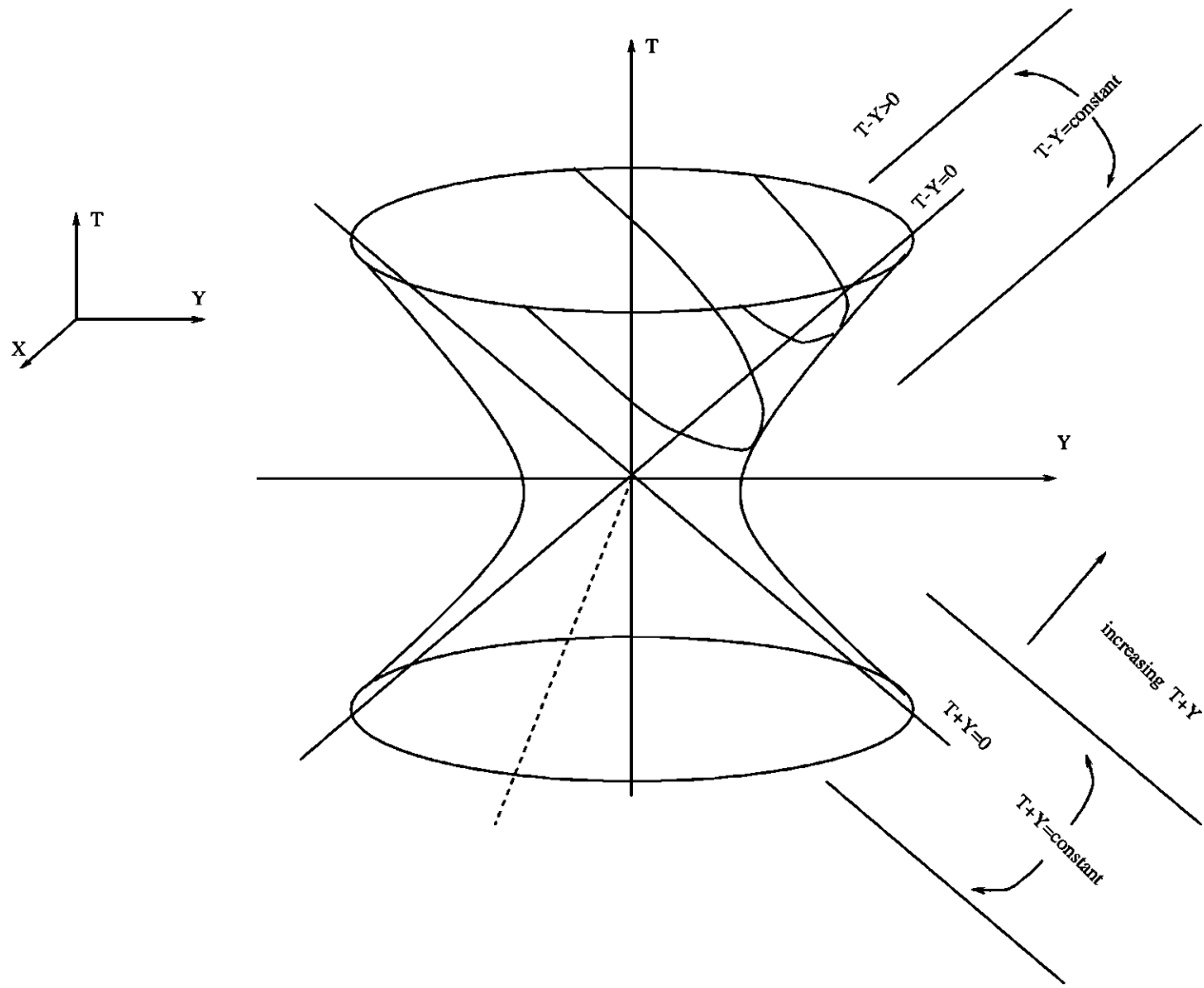

FIG. 8. The shape of the hypersurface $\alpha=\alpha_{0}$ in the new coordinates $\left(T, Y, X^{1}, X^{2}, X^{3}\right)$. Two of the coordinates, $X^{2}$ and $X^{3}$, are suppressed.
See Appendix C for details. ${ }^{6}$

We now discuss some properties of the coordinate transformation. From Eq. (106), the original coordinates $\left(t, y, x^{1}, x^{2}, x^{3}\right)$ cover only the region $Y^{2}+\mathbf{X}^{2}>T^{2}$ of the full spacetime. If we define $R=\sqrt{\mathbf{X}^{2}+Y^{2}}$, the metric can also be written as

$$
d s^{2}=\frac{4 / k_{i}^{2}}{\left(1+T^{2}-R^{2}\right)^{2}}\left[-d T^{2}+d R^{2}+R^{2} d \Omega_{3}^{2}\right]
$$

where $d \Omega_{3}^{2}$ is the metric on the unit three sphere. The conformal factor diverges at the hypersurface $R=\sqrt{1+T^{2}}$, which is the timelike boundary at infinity of the spacetime. The Penrose diagram is Fig. 20 (ii) of Ref. [33].

Another useful coordinate system is given by $R$ $=\alpha \cosh \psi, T=\alpha \sinh \psi$, in which the metric takes the form

$$
d s^{2}=\frac{4 / k_{i}^{2}}{\left(1-\alpha^{2}\right)^{2}}\left[-\alpha^{2} d \psi^{2}+d \alpha^{2}+\alpha^{2} \cosh ^{2} \psi d \Omega_{3}^{2}\right] .
$$

${ }^{6}$ We note that another conformally flat coordinate system is obtained from the transformation

$$
\bar{T}=\frac{e^{-H t}}{H \tanh \left[k_{i}\left(y-y_{i}\right)\right]}, \quad \bar{Y}=\frac{e^{-H t}}{H \sinh \left[k_{i}\left(y-y_{i}\right)\right]} .
$$

In the coordinates $\left(\bar{T}, \bar{Y}, x^{1}, x^{2}, x^{3}\right)$ the metric takes the form

$$
d s^{2}=\frac{1}{k_{i}^{2} \bar{Y}^{2}}\left[-d \bar{T}^{2}+d \bar{Y}^{2}+\left(d x^{1}\right)^{2}+\left(d x^{2}\right)^{2}+\left(d x^{3}\right)^{2}\right]
$$

The domain of the original coordinate system $\left(t, y, x^{1}, x^{2}, x^{3}\right)$ is the region $|\bar{T}|>|\bar{Y}|$.
The range of these coordinates is $0<\alpha<\infty$ and $-\infty<\psi$ $<\infty$. This coordinate system covers only the region $R<|T|$ of the full spacetime. From Eq. (106), the coordinate $\alpha$ is related to the original coordinates $t, y, x^{i}$ by

$$
\alpha=\left|\tanh \left[k_{i}\left(y-y_{i}\right) / 2\right]\right| .
$$

Consider now the hypersurface $\alpha=\alpha_{0}$, where $\alpha_{0}>0$ is a constant (see Fig. 8). In the coordinates $\left(T, Y, X^{1}, X^{2}, X^{3}\right)$, this hypersurface is the hyperboloid

$$
Y^{2}+\mathbf{X}^{2}-T^{2}=\alpha_{0}^{2} .
$$

From Eq. (112), the induced metric on this hypersurface is

$$
{ }^{(4)} d s^{2}=\frac{4 \alpha_{0}^{2}}{k_{i}^{2}\left(1-\alpha_{0}^{2}\right)^{2}}\left[-d \psi^{2}+\cosh ^{2} \psi d \Omega_{3}^{2}\right],
$$

which is the spatially compact $(k=+1)$, geodesically complete version of 4D de Sitter space. Using Eq. (113), we see that in the original coordinates $\left(t, y, x^{1}, x^{2}, x^{3}\right)$, the hypersurface (114) consists of the union of the two surfaces

$$
y=y_{i}+2 \tanh ^{-1}\left(\alpha_{0}\right) / k_{i}
$$

and

$$
y=y_{i}-2 \tanh ^{-1}\left(\alpha_{0}\right) / k_{i},
$$

on the right and left hand sides of the coordinate singularity at $y=y_{i}$. Thus, the two hypersurfaces (116) and (117) are in fact connected, even though they appear disconnected in the original coordinates $\left(t, y, x^{1}, x^{2}, x^{3}\right)$. The hypersurfaces (116) and (117) are mapped onto the regions $Y+T>0$ and $Y+T$ 
$<0$ respectively of the hypersurface (114). The hyperboloid $\alpha=\alpha_{0}$ is illustrated in Fig. 8. Note that the induced metric on each of the hypersurfaces (116) and (117) is, from Eq. (36), the spatially non-compact $(k=0)$, geodesically incomplete version of 4D de Sitter metric [33]. Note also that it follows from Eq. (103) that if the vector $\partial / \partial t$ is taken to be future directed for $y>y_{i}$, then it is past directed for $y<y_{i}$, just as in Rindler spacetime.

\section{B. The class of geodesically complete multibrane solutions}

The various multibrane solutions discussed in the earlier sections of this paper are geodesically incomplete. In particular, all the "branes" discussed in the earlier sections are surfaces of constant $y$, and it is clear from the discussion above that a surface of constant $y$ comprises in reality just half of a brane. Our goal in this section is to find geodesically complete extensions of those multibrane solutions, in which the "other halves" of all the branes are present, and also to elucidate the global structure of those extensions.

In attempting to find the geodesically complete extensions, it is convenient not to start with the multibrane solutions discussed earlier in the paper, but instead to start by directly constructing a class of geodesically complete multibrane solutions via cutting and pasting regions of 5D AdS space in the form (111). The metric is

$$
d s^{2}=\frac{4}{k^{2}\left(1+T^{2}-R^{2}\right)^{2}}\left[-d T^{2}+d R^{2}+R^{2} d \Omega_{3}^{2}\right],
$$

where we have written $k$ for $k_{i}$. Consider now a submanifold of this manifold given by

$$
\alpha_{1}^{2} \leqslant R^{2}-T^{2} \leqslant \alpha_{2}^{2} .
$$

We shall make use of three types of such submanifolds.

Type I, where $\alpha_{1}^{2}=-\infty$ and $0<\alpha_{2}^{2}<1$. Such regions contain the "particle horizon" $R=|T|$ and are spatially compact. They have a single 4D boundary at $\alpha=\alpha_{2}$.

Type II, where $0<\alpha_{1}^{2}<\alpha_{2}^{2}<1$, which are spatially compact. They have two 4D boundaries at $\alpha=\alpha_{1}$ and at $\alpha=\alpha_{2}$.

Type III, where $0<\alpha_{1}^{2}<1$ and $\alpha_{2}^{2}=1$, which are spatially non-compact. They have a single 4D boundary at $\alpha=\alpha_{1}$.

Each such submanifold is characterized by three numbers: the values of $\alpha_{1}^{2}, \alpha_{2}^{2}$ and of $k$.

Consider now the class of solutions to Einstein's equations that one can obtain by gluing together submanifolds of the above type at their boundaries. It is clear from the number of boundaries that each type of submanifold possesses that such solutions, if connected, must be in one of the following two classes:

Class A: The solution starts at one "end" with either at type I region or a type III region, then has one or more type II regions (with either orientation, i.e., $\alpha$ can be increasing or decreasing), and then finishes at the other "end" again with either a type I region or a type III region.

Class B: The solution has no "ends" and instead consists of one or more type II regions joined together in a circular fashion.
Additional solutions can of course be generated from these classes of solutions by performing additional identifications, and also by considering solutions with more than one connected component.

The junction conditions in any of these solutions are the same as those obtained earlier in the paper, except that they are now expressed in terms of different quantities. Continuity of the induced metric (115) across any boundary or brane requires that the quantity

$$
\frac{\alpha^{2}}{k^{2}\left(1-\alpha^{2}\right)^{2}}
$$

take the same value on both sides of the brane. The Israel junction condition is the requirement that the rescaled brane tension $q$ at any boundary is the sum of two terms, one from each submanifold on either side of the brane. The absolute value of each of these terms is

$$
\frac{k\left(1+\alpha^{2}\right)}{2 \alpha} .
$$

The sign of each of these terms is positive for the boundary of a type I region and for the second boundary $\alpha=\alpha_{2}>\alpha_{1}$ of a type II region. It is negative for the boundary of a type III region, and for the first boundary $\alpha=\alpha_{1}<\alpha_{2}$ of a type II region.

If one requires that all brane tensions be positive, then class A solutions with type III regions on both ends are disallowed, as are all class B solutions. Thus, there are two different types of solutions for positive tension branes: spatially compact class A solutions with type I regions on both ends, and spatially non-compact class A solutions with a type I region on one end and a type III region on the other end.

\section{Relation to earlier solutions}

Consider now how the above geodesically complete multibrane solutions are related to the multibrane solutions discussed earlier in the paper, which were characterized by parameters $k_{i}, L_{i}$ and $y_{i}$.

First consider the case of solutions with no particle horizons (which requires negative tension branes if the bulk regions are all $\mathrm{AdS}$ as in this paper). For these solutions, there is a simple and one-to-one correspondence between the solutions of Sec. III and those of Sec. VII B above. For a given solution $\left(k_{i}, y_{i}, L_{i}\right)$, one can obtain the geodesically complete extension as follows. For each interbrane region $L_{i-1}$ $<y<L_{i}$ where $L_{i-1}$ and $L_{i}$ are both finite, one constructs a region of type II with the corresponding parameters obtained from Eq. (113), namely $\alpha_{1}=\min \left(\hat{\alpha}_{1}, \hat{\alpha}_{2}\right) \quad$ and $\quad \alpha_{2}$ $=\max \left(\hat{\alpha}_{1}, \hat{\alpha}_{2}\right)$, where

$$
\hat{\alpha}_{1}^{2}=\tanh ^{2}\left[k_{i}\left(L_{i-1}-y_{i}\right) / 2\right]
$$

and

$$
\hat{\alpha}_{2}^{2}=\tanh ^{2}\left[k_{i}\left(L_{i}-y_{i}\right) / 2\right] .
$$


For any regions $L_{i-1}<y<L_{i}$ where one of the boundaries is at $|y|=\infty$, one similarly constructs a region of type III. Then, one glues the regions of type II and III together to get a geodesically complete solution. The junction conditions will be automatically satisfied. Each interbrane region in the original solution maps onto the subset $Y+T>0$ of a type II or type III submanifold of the full solution. Each $y=$ constant half-brane in the original solution acquires an additional half-brane in the full solution to make it a geodesically complete brane.

Now consider solutions with particle horizons. First, for interbrane regions not containing particle horizons one follows the same procedure as above to construct the corresponding portions of the maximally extended solution. Second, for each interbrane region $L_{i-1}<y<L_{i}$ containing a particle horizon $y=y_{i}$, there are two possibilities for the global structure.

One can map the entire interbrane region, including both sides of the particle horizon, into a single region of type I. Because the type I region has a single boundary $\alpha=\alpha_{2}$, this structure requires that the surfaces $y=L_{i-1}$ and $y=L_{i}$ both map onto the boundary $\alpha=\alpha_{2}$, and therefore both surfaces must be equidistant from the particle horizon at $y=y_{i}$. More generally, the entire multibrane solution must be reflection symmetric under the mapping $y-y_{i} \rightarrow-\left(y-y_{i}\right)$, since the regions $y>y_{i}$ and $y<y_{i}$ are the $Y+T>0$ and $Y+T<0$ portions of the same bulk region.

If the above property of symmetry under reflection through the particle horizon is not satisfied by the multibrane solution $\left(k_{i}, L_{i}, y_{i}\right)$, then the only possible global structure is as follows. One identifies the region $y>y_{i}$ to the right of the particle horizon with the $Y+T>0$ portion of a type I submanifold, and one identifies the region $y<y_{i}$ to the left of the particle horizon with the $Y+T<0$ portion of a different type I submanifold. Therefore the maximally extended multibrane solution can have several disconnected components.

The general structure of a multibrane solution $\left(k_{i}, L_{i}, y_{i}\right)$ without any additional identifications and without any special symmetries is therefore as follows. The spacetime can be split up into cells bounded by particle horizons, but with no particle horizons within any given cell. Then, the maximally extended solution consists of a number of different connected components, one for each cell. Since the physics of each connected component is independent from all the others, it is natural to restrict attention to solutions with only one connected component or cell.

This global structure justifies our prescription of Sec. III B for obtaining an effective 4D theory of restricting the integral over the fifth dimension to between pairs of particle horizons. The global structure also explains the fact that the Hubble constant $H\left(L_{i}\right)$ on any brane can be expressed entirely in terms of the tensions and locations of the branes in the same connected component, without reference to the detailed arrangement of external branes. For example, consider two branes in an uncompactified spacetime. Let the two branes be sandwiched between two horizons, and assume there is no horizon between the branes. Let the two branes be at $y=0$ and $L$, and let the bounding horizons be at $y=-y_{0}$ and $y=L+y_{2}$. Assume that $A(0)=1>A(L)$. The expansion rates on the two branes turn out to be

$$
\begin{aligned}
& H^{2}(0) \approx 4 q_{L}^{2}\left[1+\frac{\ln (1 / A(L))}{2 q_{L} L}\right]^{2}[A(L)]^{1+y_{2} / L} e^{-2 q_{L} y_{2}} \\
& H^{2}(L) \approx 4 q_{L}^{2}\left[1+\frac{\ln (1 / A(L))}{2 q_{L} L}\right]^{2}[A(L)]^{y_{2} / L} e^{-2 q_{L} y_{2}},
\end{aligned}
$$

where $q_{L}$ is the tension on the brane at $L$, and we have written the solution in terms of $A(L)$, which may be regarded as an observable quantity, the ratio $\left(m_{\text {Higgs }} / m_{\text {Planck }}\right)^{2}$ found on the visible brane.

For solutions with additional identifications, the existence of particle horizons can be compatible with the maximally extended solution having only one connected component. For example, consider the the two brane compactified model of Sec. V. That model has branes at $y=L_{0}, y=L_{1}$ and $y$ $=L_{2}$, but $L_{0}$ and $L_{2}$ are identified. When there is only one horizon, then the maximally extended solution is connected and starts with a type I region at one end, then has a type II region, then has another type I region at the other end. When there are two horizons the maximally extended solution has two connected components.

The global structure analysis also shows that the multibrane solutions of the earlier sections are a subset of the full set of multibrane solutions, for the following reason. In the earlier sections, it was always assumed that the value of the bulk cosmological constant was the same on both sides of any particle horizon in a given interbrane region. However, since the two sides of a particle horizon typically correspond to two different type I regions in the maximally extended solution, there is no reason to make this restriction. The most general class of multibrane solutions, when translated into the old $\left(t, y, x^{1}, x^{2}, x^{3}\right)$ coordinates, allows interbrane regions with particle horizons where the value of $k$ appears to change discontinuously as one crosses the particle horizon. The discontinuity is only apparent as the two sides of the particle horizon are not physically connected.

\section{4D NEWTON'S CONSTANT AND PARTICLE HORIZONS}

There are some issues that we like to resolve in this section: the determination of the 4D Newton's constant $G_{N}$ and when a large warp factor helps to solve the hierarchy problems. We have already assumed the some of the results of this section in earlier discussions. Here we like to clarify and justify them.

\section{A. The issue}

Naive determinations of $G_{N}$ sometimes yield different answers. In the multibrane model, we face the following issue. We know of 3 approaches to determine the 4D Newton's constant $G_{N}$ :

(i) integrate over the 5th dimension in the 5D action to obtain the low-energy effective 4D action [7], as discussed earlier; 
(ii) solve for the trapped gravity mode and determine its probability (wave function squared) on the brane [8];

(iii) calculate the Hubble constant and determine the coefficient of the 4D cosmological constant or the matter density contribution term [9].

So far in this paper, we have used the approaches (i) and (iii). In approach (iii), note that the value of $G_{N}$ depends only on the local (i.e., neighboring) properties of the brane on which we measure the value of $G_{N}$ :

$$
G_{N}=G_{5} \frac{2 k_{i} k_{i+1}}{k_{i}+k_{i+1}}
$$

for the $i$ th brane, where $8 \pi G_{5}=\kappa^{2}$. Recall that $k_{i}\left(k_{i+1}\right)$ is a function of $q_{i}$ as well as $q_{i-1}$ and $L_{i}-L_{i-1}\left(q_{i+1}\right.$ and $L_{i+1}-L_{i}$ ) (where the explicit dependence is modeldependent), so $G_{N}$ depends on neighboring properties only. Specifically, it does not depend on the total number of branes in the model. On the other hand, if we use the first approach, and naively integrate over the whole 5 th dimension (i.e., from $y=-\infty$ to $y=\infty$ in the uncompactified case), we will get a $G_{N}$ that depends on properties of all the branes in the model, specifically, on $N$, the number of branes. This is also the case in the second approach. If we normalize the gravity trapped mode over all $y$, we will typically get a $G_{N}$ that is roughly $N$ times smaller than for the single brane model. That is, naively, the answer from (i) and (ii) will be different than that from (iii).

The resolution to this puzzle is already evident in the global structure analysis just discussed, where a zero of the metric corresponds to a particle horizon. There, we see that the metric used is geodesically incomplete, so we should not integrate beyond the particle horizon. In calculating $G_{N}$ for the $i$ th brane using the first approach, we should integrate over the 5 th dimension only to the nearest particle horizon (i.e., the zero of the metric) on each of the brane. This also applies to the normalization of the trapped gravity mode (or any other mode) in the second approach. This way, the three approaches produce the same answer for $G_{N}$, as should be the case. Furthermore, $G_{N}$ and the effective cosmological constant is the same for all observers within the the same particle horizons, as pointed out earlier.

The reasoning presented below may be summarized as follows. It takes infinite time for a signal emitted from a brane to reach the particle horizon, as seen by observers on the brane. The trapped gravity mode on the brane is treated as a perturbation of the metric in the "almost" conformally flat background metric [8]. The nearest particle horizons in the $y$ coordinate map to $z= \pm \infty$ in the "conformal metric" coordinate. Thus, the normalization of the gravity mode over the entire $z$ coordinate only covers the region between particle horizons in the $y$ coordinate, so the properties of branes separated by particle horizons are treated independently (wavefunctions and so $G_{N}$ are independently normalized).

For the negative tension brane in the Randall-Sundrum model, and for the visible brane in the compactified model discussed above, a naive determination using approach (iii) yields a negative $G_{N}$, contradicting the above result. In Ap- pendix $\mathrm{D}$, we discuss the ambiguity and the problem arising in this approach.

This above result also means that we cannot compare the mass scales between branes that are separated by particle horizons. In this case, the mass hierarchy problem in this brane world scenario is no worse than the standard hierarchy problem. We can still compare mass scales between branes that are not separated by particle horizons, and between mass scales measured by observers on the same (visible) brane. This implies that solutions to the mass hierarchy problem can be addressed only between branes that are not separated by particle horizons. Our discussion below follows closely the original discussion of Refs. [7,8].

\section{B. Green's function and Newton's constant}

Instead of bringing the metric into the conformally flat form (110), we can bring the metric (36) into an almost "conformally flat" form, where the $y$ coordinate becomes the $z$ coordinate given by

$$
d z= \pm \frac{d y}{\frac{H}{k} \sinh \left[k\left(y-y_{0}\right)\right]}= \pm \frac{d\left[k\left(y-y_{0}\right)\right]}{H \sinh \left[k\left(y-y_{0}\right)\right]} .
$$

Here we have in mind that $H$ is very small, and all measurements involving $G_{N}$ are at distances much smaller than $1 / H$. This above equation can be readily integrated, and the result is

$$
H\left(z+z_{0}\right)=-\ln \tanh \frac{k}{2}\left|y-y_{0}\right|
$$

where $z_{0}$ is defined so that when $y=0$ we also have $z=0$. We choose the "- " sign so that when $y \rightarrow y_{0}$ we will have $z \rightarrow \infty$. We observe that the space between the horizons $\left(-y_{0},+y_{0}\right)$ is mapped into the entire real line in the new coordinate system. If the model contains many branes separated by horizons, each interval will be mapped into the entire real line, so we end up with a collection of disconnected spaces, each space containing one single brane. Using the above relationship between $y$ and $z$ we obtain the conformal factor of the metric:

$$
\frac{H^{2}}{k^{2}} \sinh ^{2}\left[k\left(y-y_{0}\right)\right]=\frac{H^{2}}{k^{2} \sinh ^{2}\left[H\left(z+z_{0}\right)\right]} .
$$

According to the result from $[7,34]$ we obtain the following wave function for the trapped graviton mode:

$$
\psi_{0}(z)=N\left(\frac{H}{k \sinh \left[H\left(z+z_{0}\right)\right]}\right)^{3 / 2}
$$

where $N$ is a constant that is determined by the normalization condition

$$
\int_{-\infty}^{+\infty}\left|\psi_{0}(z)\right|^{2} d z=1
$$


Calculating the integral we obtain

$$
-\frac{2 N^{2}}{k} \frac{H^{2}}{k^{2}}\left\{\frac{1}{4} \ln \frac{\sqrt{1+\frac{H^{2}}{k^{2}}}+1}{\sqrt{1+\frac{H^{2}}{k^{2}}}-1}-\frac{1}{2} \frac{\sqrt{1+\frac{H^{2}}{k^{2}}}}{\frac{H^{2}}{k^{2}}}\right\}=1 .
$$

Expanding for small $H / k$ we obtain

$$
N=\sqrt{k}\left\{1-\frac{H^{2}}{4 k^{2}}+\frac{H^{2}}{4 k^{2}} \ln \left(\frac{8 k^{2}}{H^{2}}+1\right)+O\left(\frac{H^{4}}{k^{4}}\right)\right\} .
$$

In the limit $H \rightarrow 0$ we obtain $N=\sqrt{k}$.

In order to obtain the relationship between the 5D and 4D Newton constants, we first note that the relation between the physical perturbation and the trapped gravity wave function is [34]

$$
\left.h_{\mu \nu}\right|_{\text {physical }}(x, z) \propto \hat{h}_{\mu \nu}(x) A^{(2-d) / 4} A(z) \psi_{0}(z) .
$$

It turns out that $\psi_{0}(z) \propto A^{(d-2) / 4}$ for this mode, so that the physical perturbation is simply $\hat{h}_{\mu \nu}(x) A(z)$. To get the right scaling of $G_{e f f}$ on the brane, one also has to consider the source term carefully. For a point particle confined to the $i$ th brane and at rest at $\mathbf{x}=\mathbf{x}_{0}$, only the 00 component of the energy momentum tensor is nonzero:

$$
T_{00}=\frac{M}{A\left(z_{i}\right)} \delta^{(3)}\left(\mathbf{x}-\mathbf{x}_{0}\right) \delta\left(z-z_{i}\right),
$$

where the brane is at $z=z_{i}, A(z)=A\left(z_{i}\right)$ and $M$ is the particle mass measured on the brane relative to the physical metric, $A\left(y_{i}\right) \hat{h}_{\mu \nu}$. The corresponding metric perturbation is (apart from possible exponentially small corrections $[37,35]$ )

$$
\begin{aligned}
\hat{h}_{00}(\mathbf{x}) & \simeq-\frac{2 G_{5}\left|\psi_{0}\left(z_{i}\right)\right|^{2} M}{A\left(z_{i}\right)\left|\mathbf{x}-\mathbf{x}_{0}\right|} \\
& =-\frac{2 G_{N} m}{\left|\mathbf{x}-\mathbf{x}_{0}\right|},
\end{aligned}
$$

where $m=M \sqrt{A\left(z_{i}\right)}$ is the mass of the particle in the effective $4 \mathrm{D}$ theory. (A similar analysis on this issue appeared while this paper was being completed [36].)

To see what the effective Newton constant is for branebound observers using the physical metric, $h_{\mu \nu}=A\left(z_{i}\right) \hat{h}_{\mu \nu}$, let us first change coordinates to a system in which the unperturbed 4D metric is Minkowski on the brane. Then it is clear that the mass produces a perturbation which is identical to $\hat{h}_{00}$, so all we have to do is re-express the solution found above in the new coordinate system. Since a coordinate separation $r$ corresponds to a distance $R=r\left(L_{i}\right)=r \sqrt{A\left(z_{i}\right)}$, and $m=M \sqrt{A\left(z_{i}\right)}$, we find that the perturbation is $\hat{h}_{00}$
$=-2 G_{N} A\left(z_{i}\right) M / R$, i.e., it is identical to Newton's law, but with a constant of gravitation $G_{e f f}=G_{N} A\left(z_{i}\right)$.

We would also like to know the corrections to Newton's force law that would be deduced on different branes. We find that, for two masses $M_{1}$ and $M_{2}$ separated by a distance $r\left(L_{i}\right)$ on the brane at $L_{i}$,

$$
F=-\frac{G_{N} A\left(L_{i}\right) M_{1} M_{2}}{r^{2}\left(L_{i}\right)}\left[1+\frac{2 C^{2}}{r^{2}\left(L_{i}\right)}\right]
$$

where the masses $M_{1}$ and $M_{2}$ and the distance $r\left(L_{i}\right)$ are measured with the induced metric $\gamma_{\mu \nu}$. Here we also include the correction term due to gravity $\mathrm{KK}$ modes, where $C^{2}\left(k_{i}, k_{i+1}\right)$ is a function of the bulk cosmological constants on the two sides of the Planck brane. We shall determine this function in the next section. When the the bulk cosmological constants on the two sides of the brane are equal, $C^{2}=k^{-2}$. In terms of the rescaled metric $\hat{\gamma}_{\mu \nu}, m_{i}=\sqrt{A\left(L_{i}\right)} M_{i}$ and $r\left(L_{i}\right)=r \sqrt{A\left(L_{i}\right)}$, and relative to the induced metric there is a force

$$
\hat{F}=A\left(L_{i}\right) F=-\frac{G_{N} m_{1} m_{2}}{r^{2}}\left[1+\frac{2 C^{2}}{A\left(L_{i}\right) r^{2}}\right]
$$

This reproduces the familiar Newton's gravitational law as given by the $4 \mathrm{D}$ effective theory (59). We see that the correction to Newton's force law on the visible brane becomes important at TeV scale distances. The Newton's force law in different metrics is discussed in terms of Feynman diagram in Appendix E.

Consider the scenario in Fig. 3. The above observation shows that the Newton's constant as seen by observers on each brane in Fig. 3 yields the same relation (125), even though the ratio $A(0) / A(L)$ can be exponentially large. The metric blow-ups beyond the particle horizons are unobservable to observers inside the particle horizons. In fact, $G_{N}$ as seen by observers on the two branes in the $S^{1} / \mathbb{Z}_{2}$ orbifold model will have the same value. By now, the reason is clear. In changing to the almost "conformally flat" metric, we have to determine $G_{N}$ separately for each brane, with each region between the horizons being mapped into real line, $-\infty<z$ $<+\infty$. Each region will contain a trapped graviton, and they do not "communicate" with each other across the particle horizon at $y=y_{0}$ since they belong to separate spaces. For an observer on the brane at $y=0$, it takes infinite time for a light-like signal to travel from the brane to $y_{0}$ :

$$
\Delta t=\frac{1}{H}\left\{\ln \left[\tanh \frac{k}{2}\left(y_{0}-y_{\text {initial }}\right)\right]-\ln \left[\tanh \frac{k}{2}\left(y_{0}-y_{\text {final }}\right)\right]\right\}
$$

where $y_{\text {initial }}=0$ and $\Delta t$ is clearly divergent when $y_{\text {final }}$ $\rightarrow y_{0}$. In terms of the 5D parameters $\kappa^{2}$ and $q_{i}$, we see that $A(0) / A(L)$ can be exponentially large. Since this huge factor is not measurable by observers on either brane, it has nothing to do with the hierarchy problem, that is, it neither solves nor worsens the standard hierarchy problem. 


\section{CORRECTIONS TO NEWTON'S GRAVITATIONAL LAW}

In the two brane model where both the hierarchy and the cosmological constant problems are solved, we note that the bulk cosmological constants on the two sides of the Planck brane are generically different. Here we consider this asymmetric situation, in particular the correction to Newton's gravitational law and the leading post-Newtonian effects. Let us summarize the key observation. The results are similar to that in the symmetric case, that is, the leading order postNewtonian effects are the same as that from Einstein theory, and the correction to Newton's gravitational law is still $r^{-2}$ as in the symmetric case [8], with a different coefficient. We shall calculate this coefficient below. Since we consider the case where the effective 4D cosmological constant is exponentially small, it is safe to ignore it (i.e., set it to zero) in most applications. This simplifies the analysis. The analysis below follows closely that of Randall and Sundrum [8] and that of Garriga and Tanaka [37-39]. Once we have the Green's function, we can also determine the correction to Newton's law for masses on the visible brane. Although the 5 th dimension is compactified in the model, the gravity $\mathrm{KK}$ spectrum is still expected to be continuous because the model has a particle horizon.

The Planck brane at $y=0$ is a surface layer separating two AdS spaces with different cosmological constants. We want to change the coordinates to Gaussian normal coordinates so that we can apply Israel's junction conditions. We expect the required change to be of the order of the perturbation induced by the mass located on the brane. Let us denote the new coordinates by

$$
\bar{x}_{ \pm}^{a}=x_{ \pm}^{a}+\xi_{ \pm}^{a}\left(x^{a}\right) .
$$

In general we will have to make different changes in coordinates for the two sides of the brane, so from now on we will suppress the \pm . In the new coordinates the metric will be

$$
d s^{2}=\bar{g}_{\mu \nu} d \bar{x}^{\mu} d \bar{x}^{\nu}+2 \bar{g}_{\mu 5} d \bar{x}^{\mu} d \bar{y}+\bar{g}_{55} d \bar{y}^{2} .
$$

The metric transforms as

$$
g_{a b}=\bar{g}_{p q} \frac{\partial \bar{x}^{p}}{\partial x^{a}} \frac{\partial \bar{x}^{q}}{\partial x^{b}}=\bar{g}_{a b}+\bar{g}_{a q} \xi_{, b}^{q}+\bar{g}_{p b} \xi_{, a}^{p}+O\left(\left(\xi^{c}\right)^{2}\right) .
$$

We include now fluctuations around this background. We want to choose the functions $\xi^{a}\left(x^{b}\right)$ so that the perturbations $h_{a b}$ satisfy the RS gauge condition while the coordinates $\bar{x}^{a}$ are Gaussian normal. Consequently we impose the conditions

$$
\begin{aligned}
& \bar{h}_{55}=0, \quad \bar{h}_{\mu 5}=0, \quad \bar{g}_{\mu 5}=0, \quad \bar{g}_{55}=1 \\
& h_{55}=0, \quad h_{\mu 5}=0, \quad h_{\mu}^{\mu}=0, \quad h_{\mu \nu}{ }^{, \nu}=0 .
\end{aligned}
$$

We transform now from the Gaussian normal coordinates to the Randall-Sundrum coordinates:

$$
\begin{aligned}
d s^{2}= & {\left[\bar{g}_{\mu \nu}+\bar{g}_{(\mu \mid \lambda} \xi_{, \mid \nu)}^{\lambda}+\bar{h}_{\mu \nu}\right] d x^{\mu} d x^{\nu}+2\left[\bar{g}_{\mu 5}+\bar{g}_{\mu \lambda} \xi_{, 5}^{\lambda}\right.} \\
& \left.+\bar{g}_{55} \xi_{, \mu}^{5}+\bar{h}_{\mu 5}\right] d x^{\mu} d y+\left[\bar{g}_{55}+2 \bar{g}_{55} \xi_{, 5}^{5}+\bar{h}_{55}\right] d y^{2} \\
= & {\left[g_{\mu \nu}+h_{\mu \nu}\right] d x^{\mu} d x^{\nu}+2\left[g_{\mu 5}+h_{\mu 5}\right] d x^{\mu} d y } \\
& +\left[g_{55}+h_{55}\right] d y^{2} .
\end{aligned}
$$

The condition $h_{55}=0$ imposes $\xi_{, 5}^{5}=0$ so this function can depend only on the coordinates parallel to the brane, $x^{\rho}$. The condition $h_{\mu 5}=0$ imposes the condition

$$
\bar{g}_{\mu \lambda} \xi_{, 5}^{\lambda}+\xi_{, \mu}^{5}=0 \Rightarrow \gamma_{\mu \lambda} \xi_{, 5}^{\lambda}+\xi_{, \mu}^{5}=0
$$

since we keep only first order terms. This fixes the $y$-dependence of the functions $\xi^{\mu}$; we have to impose continuity of these functions at $y=0, \xi_{+}^{\mu}(0)=\xi_{-}^{\mu}(0)$ :

$$
\xi^{\mu}=-\frac{1}{2 k} \gamma^{\mu \lambda} \xi_{\lambda}^{5}+\hat{\xi}^{\mu}\left(x^{\rho}\right)
$$

The functions $\hat{\xi}^{\mu}\left(x^{\rho}\right)$ will be fixed by choosing the harmonic gauge on the brane, while the functions $\xi^{5}\left(x^{\rho}\right)$ will be determined by the tracelessness of the RS perturbations. In the $\bar{x}^{a}$ coordinates the background metric is given by

$$
\bar{g}_{\mu \nu}=\eta_{\mu \nu} e^{-2 k\left|y+\xi^{5}\right|} \simeq \gamma_{\mu \nu}\left(1-2 k \xi^{5}+\cdots\right)
$$

so we can obtain the relationship between the perturbations on the two coordinate systems:

$$
\begin{aligned}
h_{\mu \nu} & =\bar{h}_{\mu \nu}-2 k \gamma_{\mu \nu} \xi^{5}-\frac{1}{2 k} \gamma_{(\mu \mid \lambda} \gamma^{\lambda \rho} \xi_{, \rho \mid \nu)}^{5}+\gamma_{(\mu \mid \lambda} \xi_{, \mid \nu)}^{\lambda} \\
& =\bar{h}_{\mu \nu}-2 k \gamma_{\mu \nu} \xi^{5}-\frac{1}{k} \xi_{, \mu \nu}^{5}+\hat{\xi}_{(\mu, \nu)} .
\end{aligned}
$$

Now the metric must be continuous at the brane. Expressing the metric in the Randall-Sundrum coordinates, the background metric is continuous at $y=0$, so the fluctuations must also be continuous at $y=0$. Using the fact that the functions $\xi^{\mu}$ must be continuous at $y=0$, and that $\xi^{5}$ is independent of $y$, we obtain the following condition:

$$
k_{+} \xi_{+}^{5}\left(x^{\rho}\right)=k_{-} \xi_{-}^{5}\left(x^{\rho}\right) .
$$

We can understand this condition in terms of the "branebending" effect: the bending of the brane should be the same as an observer in the bulk approaches the brane from either side, $y>0$ or $y<0$, so we can "glue" the two AdS spaces together along the brane. The relation (147) can also be understood in the following way. There are 5 physical polarizations for the 5D graviton. For the massive KK modes, this is precisely what one needs for a spin-2 particle. For the massless mode, the scalar component, if present, will contribute a Brans-Dicke-like interaction that is clearly ruled out. Fortunately, this scalar mode is gauged away by $\xi^{5}\left(x^{\rho}\right)$. Since there is only one scalar mode to be gauged away, $\xi_{ \pm}^{5}$ must be related. 
The jump condition at the brane, $\bar{y}=0$, can now be written as

$$
\begin{aligned}
& \left.\partial_{y}\left[\gamma_{\mu \nu}+\bar{h}_{\mu \nu}\right]\right|_{0+}-\left.\partial_{y}\left[\gamma_{\mu \nu}+\bar{h}_{\mu \nu}\right]\right|_{0-} \\
& =-\frac{2 \kappa^{2}}{3}\left[\sigma\left(\gamma_{\mu \nu}+\bar{h}_{\mu \nu}\right)+3 T_{\mu \nu}-T \gamma_{\mu \nu}\right]
\end{aligned}
$$

where $T_{\mu \nu}$ is the energy-momentum tensor and $T$ its trace. Using the explicit form of the background metric and the fact that the bulk cosmological constants are determined to give an (almost) flat brane, the above equation can be simplified to the following form:

$$
\begin{gathered}
\frac{1}{2}\left[\left.\partial_{y}^{-} \bar{h}_{\mu \nu}\right|_{0+}-\left.\partial_{y} \bar{h}_{\mu \nu}\right|_{0-}\right]+\left(k_{+}+k_{-}\right) \bar{h}_{\mu \nu} \\
=-\kappa\left[T_{\mu \nu}-\frac{1}{3} \gamma_{\mu \nu} T\right] .
\end{gathered}
$$

To obtain this equation we used the Csaki-Shirman solution for the brane tension $[40,26]$ :

$$
\kappa^{2} \sigma^{2}=\frac{3}{2}\left(\sqrt{\Lambda_{+}}+\sqrt{\Lambda_{-}}\right)^{2} .
$$

The following steps are the same as in the paper [37]: Combining the equation of motion for the metric perturbations and the jump condition, we obtain the Green's function:

$$
\begin{aligned}
\left\{a^{-2}\right. & \square(4)+\partial_{y}^{2}-4\left(k_{+}^{2} \theta(y)+k_{-}^{2} \theta(-y)\right) \\
& \left.+2\left(k_{+}+k_{-}\right) \delta(y)\right\} G_{R}\left(x, x^{\prime}\right) \\
= & \delta^{5}\left(x-x^{\prime}\right) .
\end{aligned}
$$

In the non-orbifolded case the Green's function will have contributions from both "symmetric" and "antisymmetric" KK modes. In the orbifolded case the "antisymmetric" modes are projected out. The tracelessness of the RandallSundrum metric fluctuations will result in the following equation for the $\hat{\xi}_{ \pm}^{5}\left(x^{\rho}\right)$ functions:

$$
\begin{aligned}
\square^{(4)}\left[\hat{\xi}_{+}^{5}+\hat{\xi}_{-}^{5}\right] & =\frac{1}{3} \kappa^{2} T \Rightarrow \square^{(4)}\left[k_{+} \hat{\xi}_{+}^{5}+k_{-} \hat{\xi}_{-}^{5}\right] \\
& =2 \kappa^{2} \frac{k_{+} k_{-}}{k_{+}+k_{-}} \frac{T}{3} .
\end{aligned}
$$

We will use this result later when we calculate the corrections to Newton's law. The other four functions, $\hat{\xi}^{\mu}\left(x^{\rho}\right)$ provide the gauge freedom needed to restore the 4D linearized Einstein equations. We will choose the usual harmonic gauge [41],

$$
\partial^{\mu}\left(\bar{h}_{\mu \nu}-\frac{1}{2} \eta_{\mu \nu} \bar{h}_{\lambda}^{\lambda}\right)=0
$$

so that the linearized Einstein equations for different components of the metric will decouple. Notice that the harmonic gauge condition is satisfied "up to KK corrections." The contribution from $\hat{\xi}_{ \pm}^{5}\left(x^{\rho}\right)$ will change the numeric coefficient of $T_{\lambda}^{\lambda}$ from $\frac{1}{3}$ to $\frac{1}{2}$ so that the linearized Einstein equations become

$$
\square^{(4)}\left[\bar{h}_{\mu \nu}-\frac{1}{2} \eta_{\mu \nu} \bar{h}_{\lambda}^{\lambda}\right]=-2 \kappa^{2}\left[T_{\mu \nu}-\frac{1}{2} \eta_{\mu \nu} T\right] .
$$

The setup consists of a single positive tension brane placed at $z=0$ and the bulk is made of two slices of $\operatorname{AdS}_{5}$ with different cosmological constants, $\Lambda_{i}$. The background metric can be expressed as

$$
\begin{aligned}
d s^{2} & =e^{-B(z)}\left[\eta_{a b} d x^{a} d x^{b}+d z^{2}\right] \\
& =\frac{1}{\left(k_{i} z+1\right)^{2}}\left[\eta_{a b} d x^{a} d x^{b}+d z^{2}\right] .
\end{aligned}
$$

On each side of the brane, the RS graviton satisfies the usual Schroedinger-like equation,

$$
-\partial_{z}^{2} \psi(z)+V(z) \psi(z)=m^{2} \psi(z)
$$

where

$$
\begin{aligned}
& V(z)=\frac{9}{16} B^{\prime}(z)^{2}-\frac{3}{4} B^{\prime \prime}(z) \\
& B(z)=2 \ln \left(k_{S}|z|+k_{A} z+1\right)
\end{aligned}
$$

where $k_{S}=\left(k_{-}+k_{+}\right) / 2$ and $k_{A}=\left(k_{+}-k_{-}\right) / 2$, so that the equation for the wave function becomes

$\left[-\partial_{z}^{2} \psi(z)+\frac{15\left(k_{A}+k_{S} \operatorname{sgn}(z)\right)}{4\left(k_{S}|z|+k_{A} z+1\right)^{2}}-3 k_{S} \delta(z)\right] \psi(z)=m^{2} \psi(z)$.

In order to solve the above equation, we first make the change of variable, $u=k_{i}|z|+1$ and then the change of function $\psi(u)=\sqrt{u} \chi(u)$ so that the equation becomes

$$
u^{2} \partial_{u}^{2} \chi+u \partial_{u} \chi+\left(\frac{m^{2}}{k^{2}} u^{2}-4\right) \chi=0 .
$$

The $m=0$ mode has the following solution:

$$
\psi(z)= \begin{cases}\frac{N}{\left(k_{-}|z|+1\right)^{3 / 2}}, & z<0, \\ \frac{N}{\left(k_{+}|z|+1\right)^{3 / 2}}, & z>0 .\end{cases}
$$

The normalization constant $N$ gives the 4D Newton constant,

$$
G_{N}=G e^{-B(0) / 4}|\psi(0)|^{2}=G N^{2}=G \frac{2 k_{+} k_{-}}{k_{+}+k_{-}} .
$$

In the limit $k_{+}=k_{-}=k$ we obtain the RS relationship between $G$ and $G_{N}: G_{N}=k G$. 
For the continuum mode, $m \neq 0$, the solutions of Eq. (159) are linear combinations of the Bessel functions $J_{2}\left(\left(m / k_{i}\right) u\right)$ and $Y_{2}\left(\left(m / k_{i}\right) u\right)$, so the most general solution of Eq. (159) is

$$
\psi(z)= \begin{cases}a f_{-}(z)+b g_{-}(z), & z<0, \\ c f_{+}(z)+d g_{+}(z), & z>0,\end{cases}
$$

where

$$
\begin{aligned}
& f_{ \pm}(z)=\sqrt{|z|+\frac{1}{k_{ \pm}}} J_{2}\left(m\left(|z|+\frac{1}{k_{ \pm}}\right)\right), \\
& g_{ \pm}(z)=\sqrt{|z|+\frac{1}{k_{ \pm}}} Y_{2}\left(m\left(|z|+\frac{1}{k_{ \pm}}\right)\right) .
\end{aligned}
$$

The coefficients $a, b, c$ and $d$ are determined by the continuity condition at $z=0$ and the wavefunction normalizations. The calculation is straightforward but a little tedious. The result allows us to determine the correction to the Newton's gravitational law for the Planck brane:

$$
\begin{aligned}
V(r) & \sim-\frac{G_{N} m_{1} m_{2}}{r}-\int_{0}^{\infty} \frac{G_{N}}{k} \frac{m_{1} m_{2}}{2 k} \frac{e^{-m r}}{r} d m \\
& =-\frac{G_{N} m_{1} m_{2}}{r}\left(1+\frac{C^{2}}{2 r^{2}}+\cdots\right)
\end{aligned}
$$

where

$$
C^{2}=\frac{1}{4 k_{+}^{2}} \frac{1}{1-\rho+\rho^{2}}\left[\frac{10 \rho\left(1-\rho+\rho^{2}\right)+3\left(1+\rho^{4}\right)}{3 \rho\left(1+\rho^{2}\right)+2 \rho^{2}}\right]^{2}
$$

where $\rho=k_{+} / k_{-}$. In the symmetric case $(\rho=1), C^{2}=k^{-2}$. For observers on the visible brane at $L$,

$$
V(r) \sim-\frac{G_{N} m_{1} m_{2}}{r}\left(1+\frac{C^{2}}{2 A(L) r^{2}}+\cdots\right)
$$

where $A(L) \sim 10^{-30}$ is the warp factor. The physical fluctuations of the metric will be given by: $\bar{h}_{\mu \nu}=h_{\mu \nu}+\gamma_{\mu \nu}\left[k_{+} \xi_{+}^{5}\right.$ $\left.+k_{-} \xi_{-}^{5}\right]$ where $h_{\mu \nu}$ is determined by $V(r)$. Using the relationship $G_{N}=2 k_{+} k_{-} G /\left(k_{+}+k_{-}\right)$we obtain the metric perturbations:

$$
\begin{aligned}
& \bar{h}_{00}=\frac{2 G_{N} M}{r}\left(1+\frac{2 C^{2}}{3 A(L) r^{2}}\right), \\
& \bar{h}_{i j}=\frac{2 G_{N} M}{r}\left(1+\frac{C^{2}}{3 A(L) r^{2}}\right) \delta_{i j}
\end{aligned}
$$

where $L$ can be 0 or $L_{i}$ These solutions differ from the ones obtained by expanding the Schwarzschild solution to linear order by a coordinate transformation.

\section{$\mathrm{X}$. DISCUSSIONS}

The viewpoint developed in this paper leads to a connection between the cosmological constant and mass hierarchy problems, resulting, rather generally, in relationships of the form $\left(H / m_{\text {Planck }}\right)^{2} \sim\left(m_{\text {Higgs }} / m_{\text {Planck }}\right)^{2 p}$, with $p>1$, when the expansion of the Universe is dominated by the cosmological constant. Such a relationship is reminiscent of the so-called "large numbers" of cosmology, which have been variously either dismissed as numerology or regarded as physically significant clues to some fundamental connection between the microphysical world and cosmology. Most explanations of how these numbers could arise from physics have tended to invoke variations on the theory of 4D gravitation, such as advocated by Dirac [42] or Brans and Dicke [43]. However, Zeldovich [44] proposed a different idea, that it is the cosmological constant, $\Lambda_{e f f}$, not $H$ that should appear in the large numbers, and that they therefore tell us something fundamental about the relationship of $\Lambda_{\text {eff }}$ to the rest of physics. The viewpoint developed here is intermediate, since it is based on multibrane solutions in 5D gravitation, but only involves static spacetimes. In the context of such solutions, $\ln \left(m_{\text {Planck }}^{2} / m_{\text {Higgs }}^{2}\right)$ depends on some combination of dimensionless separations between branes, and $\ln \left(m_{\text {Planck }}^{4} / \Lambda_{\text {eff }}\right)$ depends on a different combination of the separations, and is always larger than $\ln \left(m_{\text {Planck }}^{2} / m_{\text {Higgs }}^{2}\right)$. The values of these dimensionless numbers depend on the particular arrangements of branes, which are not determined in these static models, and we do not yet understand what determines these arrangements fundamentally. Moreover, even if we regard $\ln \left(m_{\text {Planck }}^{2} / m_{\text {Higgs }}^{2}\right)$ as observable, so one combination of brane displacements is knowable, the other combination, $\ln \left(m_{\text {Planck }}^{4} / \Lambda_{\text {eff }}\right)$, depends on a different combination, and is not fixed. It is clear that, for dynamical spacetimes, we should expect some movement of the branes, resulting in variability of both $\ln \left(m_{\text {Planck }}^{4} / \Lambda_{\text {eff }}\right)$ and $\ln \left(m_{\text {Planck }}^{2} / m_{\text {Higgs }}^{2}\right)$. For some reason, which we do not address here, the branes must have been relatively stationary since before the epoch of cosmological nucleosynthesis, avoiding, in particular, changes in $\ln \left(m_{\text {Planck }}^{2} / m_{\text {Higgs }}^{2}\right)$ that could alter the predicted light element abundances.

In a $4 \mathrm{D}$ spacetime theory, the cosmological constant includes contributions from microphysics, which has many scales. For example, among other contributions, supersymmetry breaking around the electroweak scale will introduce a cosmological constant of the order of $\mathrm{TeV}^{4}$, which is many orders of magnitude bigger than the value observed, around $\Lambda_{\text {eff }} \sim\left(10^{-3} \mathrm{eV}\right)^{4}$. This is the cosmological constant problem. This problem will generically persist if we consider a higher-dimensional theory and compactify it to $4 \mathrm{D}$, because the resulting effective 4D theory will face the same finetuning problem. This problem may be avoided by considering higher dimensional theory with non-factorizable geometry, and the Randall-Sundrum model is the simplest example. In fact, the RS model has an (exponential) warp factor, which provides a solution to the well-known hierarchy problem. In Ref. [4], the RS model is extended to solve the cosmological constant problem. A key feature of the model is that the effective 4D cosmological constant seen by 
an observer on the brane is not the vacuum energy density on the brane, but a non-linear combination of the brane tension and the bulk cosmological constants on the two sides of the brane. If other branes are relatively far from the visible brane, that non-linear combination becomes exponentially small, and the observer thinks he or she is seeing an exponentially small cosmological constant. For observers on the brane, it is natural to find an effective $4 \mathrm{D}$ description. Then one may ask what happens to the argument for the need of fine-tuning in $4 \mathrm{D}$ theory? The issue turns out to be quite interesting. Let us consider the single brane case first. If one insists on a 4D description for observers on the brane, one ends up with a novel description via the AdS-CFT correspondence $[10,11]$. Recall that, besides the trapped graviton, there is a continuous spectrum of Kaluza-Klein modes from 5D gravity. Thus, in addition to the standard model fields (and whatever other fields that may exist) on the visible brane in an effective 4D theory, an appropriate conformal field theory (CFT) must be included on the 3-brane, to account for the effects of the gravity KK modes. That is, the CFT is equivalent or dual to the 5D gravitational effects on the brane. This CFT interacts strongly and couples to other brane modes only via gravity. In fact, the classical correction to Newton's gravitational law in the $5 \mathrm{D}$ picture becomes a quantum effect (i.e., the CFT contribution to the vacuum polarization of the graviton) in the 4D picture [45]. Let us take the strongly interacting $S U(N) \mathbf{N}=4$ super Yang-Mills theory as the CFT. In the symmetric case, the bulk cosmological constant is related to the c-number of CFT [46,11], $2 \pi^{4} \kappa^{2} \Lambda$ $=27\left(N^{2}-1\right)$. Note that $N$ is discrete but not $\Lambda$. In this paper, we have explored the idea of adjusting the bulk cosmological constant in the context of static solutions of Einstein's equations, but it is reasonable to expect that similar adjustments occur in a dynamical spacetime. When branes move slowly, we would expect the bulk $\Lambda$ to vary slowly and continuously, perhaps just making transitions along a sequence of static models like the ones constructed here. As $\Lambda$ varies continuously, we have to consider another CFT when $2 \pi^{4} \kappa^{2} \Lambda$ $=27\left(N^{2}-1\right)$ cannot be satisfied for integer $N$. In the uncompactified multibrane model, we expect the AdS-CFT correspondence to continue to be valid. Thus, as branes move, the theory explores the space of strongly interacting CFT theories, not just the parameter space of a given quantum field theory. That is, the effective 4D theory changes as the brane separation changes. One should no longer apply here the argument for the need of fine-tuning inside a given 4D theory to obtain a very small $4 \mathrm{D}$ cosmological constant.

Finally, we note that the background field strength is normally quantized in compact models, just as momemtum is quantized on a compactified circle. However, in the models discussed here, the background field strength is not quantized due to the presence of particle horizons. Light-like signals take an infinite amount of time to reach the particle horizon, as percieved by observers on the brane. It follows that both the gravitational Kaluza-Klein spectrum (see Secs. VIII and IX) and the value of the background field strength are continuous.

\section{SUMMARY AND REMARKS}

In this paper we argue that, for parallel 3-branes that are relatively far apart, an exponentially small cosmological constant is quite generic. We present a two brane model which solves the hierarchy problem in the manner of Randall and Sundrum and has an exponentially small cosmological constant for observers on the visible brane. Moreover, the cosmological constant, in Planck units, turns out to be much smaller than the Higgs boson mass in Planck units, naturally and generically. To be explicit, we write down the 4D low energy effective action for the model. We consider this model as another motivation for the brane world.

We see that the inequality (4) is robust. This follows from the fact that both the visible brane and the Planck brane must be inside the same particle horizons. The cosmological constant is exponentially small roughly as a function of the distance of the Planck brane from the particle horizon while the hierarchy factor is exponentially small roughly as a function of the distance of the Planck brane from the visible brane. Since the visible brane must be between the Planck brane and the particle horizon, the inequality (4) follows.

We discuss the physical implications of the particle horizons that may appear in the AdS bulk. In the process, we clarify some confusing issues in the literature. We also discuss a possible "phase transition" in the two brane model, which deserves more attention. We have concentrated exclusively on bulks that are purely AdS. Similar, simultaneous explanations for the cosmological constant and mass hierarchy problems may also be possible when the bulks are not pure AdS; this question also needs further study.

There are interesting questions that remain to be addressed. Are the solutions stable under quantum effects? We believe so. Both the hierarchy problem and the cosmological constant problems are solved by involving factors that are exponential functions of brane separations, so they become exponentially small as brane separation becomes large. This behavior resembles the Yukawa force, which is robust under quantum corrections. This leads us to believe that quantum corrections will not destroy the exponential behaviors in the brane world.

It will be interesting to find a reason why the 3 -branes like to stay parallel. The dynamics of these brane world models will be important. String or M theory realization is another important question.

\section{ACKNOWLEDGMENTS}

We thank Philip Argyres, Gia Dvali, Zurab Kakushadze, Juan Maldacena, Vatche Sahakian and Adrian Salzmann for discussions. This research is partially supported by NSF (S.H.H.T. and E.E.F.) and NASA (I.W.).

\section{APPENDIX A: THE STATIC SOLUTION TO THE BRANE EQUATION OF MOTION}

We can now check that the metric (42) satisfies all the equations of motion derived from the action (10). The Einstein equations, the equation of motion of the field $A_{a_{1} \cdots a_{d-1}}$, and the equation for the induced metric have al- 
ready been checked. Here we check the equation for the embedding coordinates, i.e., the brane equation of motion, Eq. (14). In this appendix, we shall express the brane equation of motion in terms of the metric (36). There are three types of embedding coordinates, the spatial coordinates tangential to the brane, the time coordinate and the coordinate normal to the brane. We consider them separately.
The $a=j=1,2,3$ case. This case is trivial, since all the terms in Eq. (14) are zero. The last term contains the fieldstrength tensor with two indices repeated: $F_{\mu_{1} \cdots \mu_{4}}^{i} \epsilon^{\mu_{1} \cdots \mu_{4}}$ and is zero due to the antisymmetry of the tensor. The first term can also be proven to be zero. The embedding coordinates are scalars with respect to the intrinsic coordinates of the brane, so we obtain

$$
\nabla^{\mu} \nabla_{\mu} \xi_{n}^{j}=\frac{1}{\sqrt{\left|\gamma_{n}\right|}} \partial^{\mu}\left[\sqrt{\left|\gamma_{n}\right|} \partial_{\mu} \xi_{n}^{j}\right]=\frac{1}{\sqrt{\left|\gamma_{n}\right|}} \partial^{j}\left[\sqrt{\left|\gamma_{n}\right|}\right]=0
$$

since $\left|\gamma_{n}\right|$ depends only on $\xi^{0}$ and $Y_{n}$. We now prove that the second term of the equation is also zero:

$$
\begin{aligned}
\Gamma_{a b}^{j} \partial_{\alpha} X^{a} \partial_{\beta} X^{b} \gamma_{n}^{\alpha \beta} & =\Gamma_{a b}^{j} \delta_{\alpha}^{a} \delta_{\beta}^{b} \gamma_{n}^{\alpha \beta}=\Gamma_{\alpha \beta}^{j} g^{\alpha \beta}=\frac{1}{2} g^{j a}\left[\frac{\partial g_{a \beta}}{\partial \xi^{\alpha}}+\frac{\partial g_{\alpha a}}{\partial \xi^{\beta}}-\frac{\partial g_{\alpha \beta}}{\partial x^{a}}\right] g^{\alpha \beta} \\
& =\frac{1}{2} g^{j j}\left[\frac{\partial g_{j \beta}}{\partial \xi^{\alpha}}+\frac{\partial g_{\alpha j}}{\partial \xi^{\beta}}\right] g^{\alpha \beta}=g^{j j} \sum_{\alpha} \frac{\partial g_{j \alpha}}{\partial \xi^{\alpha}}=g^{j j} \frac{\partial g_{j j}}{\partial \xi^{j}}=0
\end{aligned}
$$

since the metric is diagonal and does not depend on $\xi^{j}$.

The case $a=0$. In this case the term containing the field strength tensor is also zero due to repeated indices and antisymmetry, but the first two terms are no longer zero. However, it will turn out that the two terms will cancel each other. The first term can be easily calculated:

$$
\frac{1}{\sqrt{\left|\gamma_{n}\right|}} \partial^{0}\left[\sqrt{\left|\gamma_{n}\right|} \partial_{0} X^{0}\right]=\frac{g^{00}}{\sqrt{\left|\gamma_{n}\right|}} \partial_{0}\left[\sqrt{\left|\gamma_{n}\right|}\right]=\frac{g^{00}}{\sqrt{\left|g\left(L_{n}\right)\right|}} \partial_{0}\left[\sqrt{\left|g\left(L_{n}\right)\right|}\right] .
$$

In terms of the metric (36), this is equal to $-4 H / A\left(L_{n}\right)$. The second term can be calculated as in the previous case:

$$
\begin{aligned}
\Gamma_{\alpha \beta}^{0} g^{\alpha \beta} & =\frac{1}{2} g^{0 a}\left[\frac{\partial g_{a \beta}}{\partial \xi^{\alpha}}+\frac{\partial g_{\alpha a}}{\partial \xi^{\beta}}-\frac{\partial g_{\alpha \beta}}{\partial \xi^{a}}\right] g^{\alpha \beta} \\
& =\frac{1}{2} g^{00}\left[\frac{\partial g_{0 \beta}}{\partial \xi^{\alpha}}+\frac{\partial g_{\alpha 0}}{\partial \xi^{\beta}}\right] g^{\alpha \beta}-\frac{1}{2} g^{00} \frac{\partial g_{\alpha \beta}}{\partial \xi^{0}} g^{\alpha \beta} \\
& =\frac{1}{2} g^{00}\left[2 \frac{\partial g_{00}}{\partial \xi^{0}}\right]-\frac{1}{2} g^{00} \frac{1}{g} \frac{\partial g}{\partial \xi^{0}}=-g^{00} \frac{1}{\sqrt{|g|}} \frac{\partial \sqrt{|g|}}{\partial \xi^{0}}
\end{aligned}
$$

which equals $4 H / A\left(L_{n}\right)$ in the metric (36). The two terms cancel each other, so this equation is identically satisfied.

The case $a=5$. If $a=5$ the term containing the field strength tensor is no longer zero, so we calculate this term first:

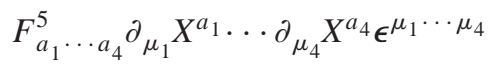

$$
\begin{aligned}
& =F_{\mu_{1} \cdots m_{m} u_{4}}^{5} \epsilon^{\mu_{1} \cdots \mu_{4}}=g^{5 a} F_{a \mu 1 \cdots \mu_{4}} \epsilon^{\mu_{1} \cdots \mu_{4}}=4 ! F_{50123}=4 ! F_{01235} .
\end{aligned}
$$

The equation of motion for the field $A$ has already been solved, and the solution is

$$
F^{a_{1} \cdots a_{5}}=\frac{e}{\sqrt{(|g|)}} \epsilon^{a_{1} \cdots a_{5}}
$$

We can use this result to calculate the value of $F_{01235}$ : 


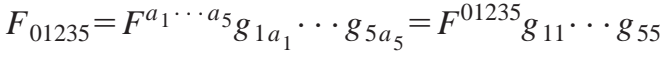

$$
\begin{aligned}
& =g F^{01235}=-\sqrt{|g|} e \epsilon^{01235}=-e \sqrt{|g|} .
\end{aligned}
$$

The second term is

$$
\begin{aligned}
\Gamma_{a b}^{5} \partial_{\alpha} X^{a} \partial_{\beta} X^{b} \gamma^{\alpha \beta} & =\Gamma_{\alpha \beta}^{5} g^{\alpha \beta}=\frac{1}{2} g^{5 a}\left[\frac{\partial g_{a \beta}}{\partial \xi^{\alpha}}+\frac{\partial g_{\alpha a}}{\partial \xi^{\beta}}-\frac{\partial g_{\alpha \beta}}{\partial \xi^{a}}\right] g^{\alpha \beta} \\
& =-\frac{1}{2} g^{\alpha \beta} \frac{\partial g \alpha \beta}{\partial y}=-\frac{1}{2} \frac{1}{g} \frac{\partial g}{\partial y}=-\frac{1}{\sqrt{|g|}} \frac{\partial \sqrt{|g|}}{\partial y} .
\end{aligned}
$$

Finally using the fact that $\sqrt{\left|\gamma_{n}\right|}=\sqrt{\left|g\left(L_{n}\right)\right|}$, the equation becomes

$$
\sigma_{n} \frac{\partial \sqrt{|g|}}{\partial y}+e_{n} e \sqrt{|g|}=0 .
$$

In the case where the derivative of $g_{\mu \nu}$ has a jump across the brane, we should take the average of the values on the two sides. This yields Eq. (39). Naively, we expect this equation to impose a non-trivial constraint between the brane tension and the brane charge, but in the static case we are interested in, this equation will be identically satisfied if the Einstein equation is satisfied.

A similar result can be obtained for the brane equation of motion in the other models discussed in Sec. II.

\section{APPENDIX B: DETAILS OF THE TWO BRANE MODEL}

Using the continuity and junction conditions (81),(82), we can solve for each of the coth functions in Eq. (83) in terms of $k_{1}, k_{2}, q_{0}, q_{1}$, giving

$$
\begin{aligned}
& \operatorname{coth}\left[k_{1}\left(L_{0}-y_{1}\right)\right]=\frac{k_{2}^{2}-k_{1}^{2}-q_{0}^{2}}{2 q_{0} k_{1}}, \\
& \operatorname{coth}\left[k_{1}\left(L_{1}-y_{1}\right)\right]=\frac{k_{1}^{2}-k_{2}^{2}+q_{1}^{2}}{2 q_{1} k_{1}}, \\
& \operatorname{coth}\left[k_{2}\left(L_{1}-y_{2}\right)\right]=\frac{k_{1}^{2}-k_{2}^{2}-q_{1}^{2}}{2 q_{1} k_{2}}, \\
& \operatorname{coth}\left[k_{2}\left(L_{2}-y_{2}\right)\right]=\frac{k_{2}^{2}-k_{1}^{2}+q_{0}^{2}}{2 q_{0} k_{2}} .
\end{aligned}
$$

Following from Fig. 2 and the subsequent discussion, the possible values of the various coth functions can be deduced. Using Eq. (B1), these inequalities are constraints on the allowed values of $k_{1}$ and $k_{2}$, given $q_{0}$ and $q_{1}$. Here $\operatorname{coth}\left[k_{1}\left(L_{0}-y_{1}\right)\right] \leqslant-1$ and $\operatorname{coth}\left[k_{2}\left(L_{2}-y_{2}\right)\right] \geqslant 1$ always for positive tension branes, which imply $k_{2}^{2} \leqslant\left(k_{1}-q_{0}\right)^{2}$ and $k_{1}^{2}$ $\leqslant\left(k_{2}-q_{0}\right)^{2}$ respectively. The other inequalities depend on the behavior of the scale factor in the bulks between the branes.

If there is a horizon between $L_{1}$ and $L_{0}$ only,

$$
\begin{aligned}
& \operatorname{coth}\left[k_{1}\left(L_{1}-y_{1}\right)\right] \geqslant 1 \Rightarrow k_{2}^{2} \leqslant\left(k_{1}-q_{1}\right)^{2}, \\
& \operatorname{coth}\left[k_{2}\left(L_{1}-y_{2}\right)\right] \geqslant 1 \Rightarrow k_{1}^{2} \geqslant\left(k_{2}+q_{1}\right)^{2} ;
\end{aligned}
$$

if there is a horizon between $L_{2}$ and $L_{1}$ only,

$$
\begin{aligned}
& \operatorname{coth}\left[k_{1}\left(L_{1}-y_{1}\right)\right] \leqslant-1 \Rightarrow k_{2}^{2} \geqslant\left(k_{1}+q_{1}\right)^{2}, \\
& \operatorname{coth}\left[k_{2}\left(L_{1}-y_{2}\right)\right] \leqslant-1 \Rightarrow k_{1}^{2} \leqslant\left(k_{2}-q_{1}\right)^{2} ;
\end{aligned}
$$

if there is a horizon in both bulks,

$$
\begin{gathered}
\operatorname{coth}\left[k_{1}\left(L_{1}-y_{1}\right)\right] \geqslant 1 \Rightarrow k_{2}^{2} \leqslant\left(k_{1}-q_{1}\right)^{2}, \\
\operatorname{coth}\left[k_{2}\left(L_{1}-y_{2}\right)\right] \leqslant-1 \Rightarrow k_{1}^{2} \leqslant\left(k_{2}-q_{1}\right)^{2} .
\end{gathered}
$$

The allowed regions of the " $k$-plane" can be further constrained by solving Eq. (B1) for the brane separations in terms of $k_{1}$ and $k_{2}$ :

$$
\begin{aligned}
& L_{1}-L_{0}=\frac{1}{2 k_{1}} \ln \left[\frac{\left(k_{1}+k_{2}+q_{1}\right)\left(k_{1}-k_{2}+q_{1}\right)\left(k_{1}+k_{2}+q_{0}\right)\left(k_{1}-k_{2}+q_{0}\right)}{\left(k_{1}+k_{2}-q_{0}\right)\left(k_{1}-k_{2}-q_{0}\right)\left(k_{1}+k_{2}-q_{1}\right)\left(k_{1}-k_{2}-q_{1}\right)}\right] \\
& L_{2}-L_{1}=\frac{1}{2 k_{2}} \ln \left[\frac{\left(k_{2}+k_{1}+q_{1}\right)\left(k_{2}-k_{1}+q_{1}\right)\left(k_{2}+k_{1}+q_{0}\right)\left(k_{2}-k_{1}+q_{0}\right)}{\left(k_{2}+k_{1}-q_{1}\right)\left(k_{2}-k_{1}-q_{1}\right)\left(k_{2}+k_{1}-q_{0}\right)\left(k_{2}-k_{1}-q_{0}\right)}\right] .
\end{aligned}
$$


When there is no horizon between $L_{0}$ and $L_{1}, L_{2}-L_{1}$ is positive in the region allowed by Eq. (B3), but the condition $L_{1}-L_{0} \geqslant 0$ requires that $k_{2}^{2} \geqslant k_{1}^{2}+q_{0} q_{1}$. There is a reflection of this constraint about the line $k_{1}=k_{2}$ in order for $L_{2}-L_{1}$ $\geqslant 0$ when there is no horizon in the second bulk. Finally, we have imposed $L_{2}-L_{1} \geqslant L_{1}-L_{0}$ to distinguish the physically distinct configurations of the branes and horizons between them. The function $\left(L_{2}-L_{1}\right)-\left(L_{1}-L_{0}\right)$, obtained from Eq. (B5), can be evaluated numerically, revealing the regions in which this last condition is satisfied. Combining all constraints gives Fig. 7.

We find in Sec. V that when there is a horizon in the second bulk, in the limit of large $\Delta l_{1,2}$, the hierarchy scale factor is $m_{\text {Higgs }}^{2} / m_{\text {Planck }}^{2} \sim \exp \left[-2 \Delta l_{1}\right]$, and the cosmological constant scale factor is $\Lambda_{\text {eff }} / m_{\text {Planck }}^{4} \sim \exp \left[-\left(\Delta l_{1}+\Delta l_{2}\right)\right]$ (with a horizon in the first bulk only, the roles of $\Delta l_{1}$ and $\Delta l_{2}$ will be reversed). We now prove that in our model, the hierarchy scale factor is always greater than that for the cosmological constant. The curve along which $\Delta l_{2}=\Delta l_{1}$ can be obtained from Eq. (B5) most simply by defining $s=k_{2}+k_{1}$ and $t=k_{2}-k_{1}$. The condition $\Delta l_{2}=\Delta l_{1}$ becomes

$$
\frac{\left(t+q_{1}\right)\left(t+q_{0}\right)}{\left(t-q_{1}\right)\left(t-q_{0}\right)}=\frac{\left(t-q_{1}\right)\left(t-q_{0}\right)}{\left(t+q_{1}\right)\left(t+q_{0}\right)}
$$

which has $t=0$ or $k_{2}=k_{1}$ as its solution for positive brane tensions. Above this line $\Delta l_{2}>\Delta l_{1}$. Then for $k_{2}>k_{1}$, the only allowed region in Fig. 7 is that for the single horizon in the second bulk; there $\Delta l_{2}>\Delta l_{1}$, making the hierarchy scale larger. For $k_{2}<k_{1}$ there are two allowed regions, one of which contains a horizon in both bulks, and hence does not provide a solution to the hierarchy problem; the other region has a horizon in the first bulk only. In that region $\Delta l_{2}$ determines the hierarchy scale and $\Delta l_{1}$ determines the cosmological constant scale, and since $\Delta l_{1}>\Delta l_{2}$, the hierarchy scale factor is again the larger.

A calculation similar to that which led to Eq. (86) reveals that, when there is a particle horizon in each of the two bulks,

$$
H^{2}\left(L_{1}\right) \sim \exp \left[-\left(\Delta l_{1}+\Delta l_{2}\right)\right]
$$

with a complicated coefficient of proportionality. The Hubble constant in such a case will be greater than that with a single horizon because $k_{2}$ is less, giving a smaller $\Delta l_{2}$; this is evident from Fig. 7. Hence the single horizon model is an energetically favorable configuration, if such a comparison is meaningful.

\section{APPENDIX C: DETAILS OF COORDINATE TRANSFORMATION}

In this appendix we describe the derivation of the coordinate transformation (103)-(106). We start by scaling out all the dimensional constants by defining $\hat{t}=H t, \hat{y}=k_{i}\left(y-y_{i}\right)$, and $\hat{x}^{l}=H x^{l}$. The metric (101) can now be written as

$$
k_{i}^{2} d s^{2}=d \hat{y}^{2}-\sinh ^{2} \hat{y} d \hat{t}^{2}+\sinh ^{2} \hat{y} e^{2 \hat{t}} d \hat{\mathbf{x}}^{2} .
$$

If we focus attention on the first two terms in this line element, we see that the coordinate singularity at $\hat{y}=0$ is analogous to the coordinate singularity of the two dimensional Rindler metric $-y^{2} d t^{2}+d y^{2}$, and can be eliminated by the coordinate transformation

$$
\begin{aligned}
& \bar{y}=\tanh (\hat{y} / 2) \cosh \hat{t} \\
& \bar{t}=\tanh (\hat{y} / 2) \sinh \hat{t} .
\end{aligned}
$$

The metric now takes the form $k_{i}^{2} d s^{2}=\Omega^{2} d \bar{s}^{2}$, where

$$
\Omega^{2}=\frac{4}{\left(1-\bar{y}^{2}+\bar{t}^{2}\right)^{2}}
$$

and

$$
d \bar{s}^{2}=-d \bar{t}^{2}+d \bar{y}^{2}+(\bar{t}+\bar{y})^{2} d \hat{\mathbf{x}}^{2} .
$$

The coordinate singularity at $\hat{y}=0$ has now been eliminated in the first two terms of the metric, but persists as the coordinate singularity $\bar{t}+\bar{y}=0$ of the last term in Eq. (C4). However, the metric $(\mathrm{C} 4)$ is just 5D Minkowski spacetime. ${ }^{7}$ In double null coordinates $\bar{u}=\bar{t}+\bar{y}, \bar{v}=\bar{t}-\bar{y}$ it can be written as

$$
d \bar{s}^{2}=-d \bar{u} d \bar{v}+\bar{u}^{2} d \hat{\mathbf{x}}^{2}
$$

The transformation to Lorentzian coordinates for this flat metric can be obtained by solving for the exponential map from the tangent space at the point $\bar{u}=1, \bar{v}=0, \hat{x}^{l}=0$ to the full spacetime. This yields the coordinate transformation

$$
\begin{aligned}
U & =\bar{u} \\
V & =\bar{v}+\bar{u} \hat{\mathbf{x}}^{2} \\
X^{l} & =\bar{u} \hat{x}^{l},
\end{aligned}
$$

and the metric $(\mathrm{C} 4)$ now takes the simple form $-d U d V$ $+d \mathbf{X}^{2}$. If we now define coordinates $T$ and $Y$ by $U=T+Y$ and $V=T-Y$, and combine together all the successive coordinate transformations of this appendix, we obtain the transformations (103)-(106) and the metric (109) given in the body of the paper.

\footnotetext{
${ }^{7}$ We note that for the metric$$
k_{i}^{2} d s^{2}=d \hat{y}^{2}-A(\hat{y}) d \hat{t}^{2}+A(\hat{y}) e^{2 \hat{t}} d \hat{\mathbf{x}}^{2},
$$

if we define coordinates $\bar{y}=f(\hat{y}) \cosh \hat{t}$ and $\bar{t}=f(\hat{y}) \sinh \hat{t}$ and choose $\left(f^{\prime} / f\right)^{2}=1 / A(\hat{y})$ (where prime denotes differentiation with respect to $\hat{y}$ ), then $k_{i}^{2} d s^{2}=\left(f^{\prime}\right)^{-2} d \bar{s}^{2}$, with $d \bar{s}^{2}$ given by Eq. (C4).
} 


\section{APPENDIX D: $G_{N}$ AND $\Lambda_{e f f}$}

Here we explain why the determination of $G_{N}$ from cosmology via the Hubble constant is sometimes misleading. Recall that $H^{2}=\kappa_{N}^{2} \Lambda_{e f f} / 3$, where the 4D gravitational coupling is given by Eq. (56), while the 4D effective cosmological constant $\Lambda_{e f f}$ is given by Eq. (58),

$$
\Lambda_{e f f}=\sum A^{2}\left(L_{i}\right) \sigma_{i}-F\left(k_{i}, L_{i}\right),
$$

so both $\kappa_{N}^{2}$ and $\Lambda_{\text {eff }}$ are dependent on the positions $L_{i}$ of the branes within particle horizons and the piecewise constant $\Lambda$, or equivalently, the $k_{i}$. Since $\sigma_{i}, \kappa_{N}^{2}, L_{i}$ and $k_{i}$ are related by Einstein's equation, a perturbation on one of the brane tensions, $\sigma_{i} \rightarrow \sigma_{i}+\delta \sigma_{i}$, requires a corresponding change in some of the other quantities. This back reaction must be taken into account. Let us see when this back reaction is expected to be small and when it may be sizable. Let us first consider the single positive tension brane model, where the brane is not charged. Here, $H^{2}=\left(q^{2}-4 k^{2}\right) / 4, \kappa^{2} \Lambda_{e f f} / 3=q$ $-2 k$ and $\kappa_{N}^{2}=2 k \kappa^{2}$. We can vary the brane tension, or equivalently $q$ by a small positive amount, $q \rightarrow q+\delta q$. Keeping $k$ constant, we find that $d H^{2} / d q=q / 2>0$ and $\kappa_{N}^{2}$ does not change. This implies that the $d \Lambda_{e f f} / d q>0$. So we see that $\kappa_{N}^{2}=3 \delta H^{2} / \delta \Lambda_{e f f}$. As we shall see, a similar result will be obtained in a multibrane model if the metric is peaked at $q$ brane.

Now, let us consider a model where the hierarchy problem can be solved. Besides the visible brane, the model must involve at least another brane, where the metric factor $A(y)$ is (exponentially) bigger. To be specific, let us consider a two brane model, where $\sigma_{1}$ is the visible brane tension and $\sigma_{0}$ is the Planck brane tension

$$
\Lambda_{e f f}=\sigma_{0}+A^{2}\left(L_{1}\right) \sigma_{1}-F\left(k_{1}, k_{2}, L_{1}, L_{2}\right)
$$

where $A\left(L_{1}\right)$ is exponentially small. So the direct visible brane tension contribution to $\Lambda_{\text {eff }}$ is exponentially small, and a small change in $\sigma_{1}$ is totally negligible. However, as $\sigma_{1}$ $\rightarrow \sigma_{1}+\delta \sigma_{1}$, either $\sigma_{0}, A\left(L_{1}\right)$ or $F\left(k_{1}, k_{2}, L_{1}, L_{2}\right)$ will adjust accordingly, depending on the details of dynamics of the model. Since the influence of $\sigma_{1}$ on the other quantities are not necessarily exponentially suppressed, the induced change in $\Lambda_{\text {eff }}$ can be large. It is easy to see scenarios where increasing $\sigma_{1}$ actually causes $\Lambda_{\text {eff }}$ to decrease, or where $\Lambda_{\text {eff }}$ still increases, but $G_{N}$ decreases in a way such that $H^{2}$ decreases.

\section{APPENDIX E: NEWTON'S FORCE LAW}

Here we consider the Newton's force law between two point masses on the visible brane explicitly. An easy way is to compare it to the electric force law, since the electromagnetic field $A_{\mu}$ which is confined on the brane does not undergo a rescaling.

Let us consider the electromagnetic field and two charged scalar fields confined on the visible brane at $y=L$ :

$$
\begin{aligned}
\mathbf{S}^{(4)}= & \int d^{4} x \sqrt{-\hat{\gamma}}\left[R^{(4)} / 2 \kappa_{N}^{2}-\frac{1}{4} F_{\mu \nu} F^{\mu \nu}\right. \\
& \left.+\sum_{j} \frac{A(L)}{2} D_{\mu} \phi_{j}^{+} D^{\mu} \phi_{j}-A(L)^{2} M_{j}^{2} \phi_{j}^{2} / 2\right] .
\end{aligned}
$$

In solving the hierarchy problem, we expect $A(L) \sim 10^{-30}$. The electric charge $e$ in $D_{\mu}=\partial_{\mu}-i e A_{\mu}$ is of order unity. As before [7], we can rescale the scalar fields $\hat{\phi}_{j}=\sqrt{A(L)} \phi_{j}$ and the masses $m_{j}=\sqrt{A(L)} M_{j}(j=1,2)$ to obtain

$$
\begin{aligned}
\mathbf{S}^{(4)}= & \int d^{4} x \sqrt{-\hat{\gamma}}\left[R^{(4)} / 2 \kappa_{N}^{2}-\frac{1}{4} F_{\mu \nu} F^{\mu \nu}\right. \\
& \left.+\sum_{j} \frac{1}{2} D_{\mu} \hat{\boldsymbol{\phi}}_{j}^{+} D^{\mu} \hat{\boldsymbol{\phi}}_{j}-\frac{1}{2} m_{j}^{2} \hat{\boldsymbol{\phi}}_{j}^{2}\right]
\end{aligned}
$$

which is in the standard familiar form. Note that there is no rescaling for gauge field $A_{\mu}$. As pointed out before, the effective action is independent of the way we define the warp factor, $A(0)=1$, and $A(L)=\left(m_{\text {Higgs }} / m_{\text {Planck }}\right)^{2}$, or $A(0)$ $=\left(m_{\text {Planck }} / m_{\text {Higgs }}\right)^{2}$ and $A(L)=1$. The effective action is also invariant to any further changes in the $\left\{x^{\mu}\right\}$ coordinates. The difference in the coordinates parallel to the brane, $\Sigma_{\mu}\left(x_{a}^{\mu}-x_{b}^{\mu}\right)^{2}$, will represent the physical distance measured by an observer on the brane only if $A(L)=1$, so we may use this convention.

To warm up, let us first use the action (E2) to calculate the Newton's force law by considering the one graviton exchange between the two scalar fields in the low energy approximation with linearized gravity. In this large distance case, the energy-momentum tensor is $T_{\mu \nu} \simeq \eta_{\mu \nu} \Sigma_{j} m_{j}^{2} \hat{\phi}_{j}^{2}$ $+\cdots$. The result is well known:

$$
-G_{N} \frac{m_{1}^{2} m_{2}^{2}}{r^{2}}, \quad e^{2} \frac{m_{1} m_{2}}{r^{2}}
$$

where we also give the one-photon exchange case. Comparing to the usual electric force law $e^{2} / r^{2}$, we notice that we must factor out the $m_{1} m_{2}$ factor in the one-graviton exchange term to obtain the usual Newton's force law $-G_{N} m_{1} m_{2} / r^{2}$. This is the result in the $\left(\hat{\gamma}_{\mu \nu}, \hat{\phi}_{j}, m_{j}\right)$ frame. Suppose we use the action (E1) instead, that is, in the $\left(\gamma_{\mu \nu}, \phi_{j}, M_{j}\right)$ frame. The one-graviton exchange now gives

$$
-G_{N} \frac{\left[A(L)^{2} M_{1}^{2}\right]\left[A(L)^{2} M_{2}^{2}\right]}{r^{2}} .
$$

In the one-photon exchange case, we note that the coupling term ieA $(L) A^{\mu} \phi^{+} \partial_{\mu} \phi$ implies the electric charge in this case to be $e A(L)$. Using the Fourier transform of $A(L) \partial_{\mu} \partial^{\mu} \phi-A(L)^{2} M^{2} \phi \simeq 0$, we see that

$$
[e A(L)]^{2} \frac{\left[\sqrt{A(L)} M_{1}\right]\left[\sqrt{A(L)} M_{2}\right]}{r^{2}} .
$$


To obtain the electric force law $e^{2} / r^{2}$, we have to factor out a $M_{1} M_{2} A(L)^{3}$ factor, so the Newton's force law is given by

$$
-G_{N} \frac{A(L) M_{1} M_{2}}{r^{2}}=-G_{N} \frac{m_{1} m_{2}}{r^{2}}
$$

so we obtain the Newton's force law of the effective theory, as expected. Since both forces are proportional to $r^{-2}$, a rescaling of $r$ does not change the result.

We can also compare the relative strengths of the electric and gravitational forces between two particles:

$$
\frac{F_{G}}{F_{e l}}=-G_{N} A(L) \frac{M_{1} M_{2}}{e^{2}} .
$$

Although the gravitational force is so much weaker than the electric force in the visible brane, the ratio is increased by a factor of about $\left(m_{\text {Planck }} / m_{\text {Higgs }}\right)^{2}$ in the Planck brane. The variation of the relative strength of the forces as a function of the brane separation is analogous to the running of the couplings in ordinary field theory.
[1] A. Balbi et al., Astrophys. J. Lett. 545, L1 (2000); A.E. Lange et al., Phys. Rev. D 63, 042001 (2001); A.G. Riess et al., Astron. J. 116, 1009 (1998); S. Perlmutter et al., Astrophys. J. 517, 565 (1999); see also N.A. Bahcall, J.P. Ostriker, S. Perlmutter, and P.J. Steinhardt, Science 284, 1481 (1999).

[2] S. Weinberg, Rev. Mod. Phys. 61, 1 (1989).

[3] For additional useful reviews, see, e.g., S.M. Carroll, W.H. Press, and E.L. Turner, Annu. Rev. Astron. Astrophys. 30, 499 (1992); S. Weinberg, "The Cosmological Problems," astro-ph/0005265; E. Witten, "The Cosmological Constant from the Viewpoint of String Theory," hep-ph/0002297; S.M. Carroll, "The Cosmological Constant," astro-ph/0004075.

[4] S.-H.H. Tye and I. Wasserman, Phys. Rev. Lett. 86, 1682 (2001).

[5] N. Arkani-Hamed, S. Dimopoulos, and G. Dvali, Phys. Lett. B 429, 263 (1998).

[6] V.A. Rubakov and M.E. Shaposhnikov, Phys. Lett. 125B, 139 (1983).

[7] L. Randall and R. Sundrum, Phys. Rev. Lett. 83, 3370 (1999).

[8] L. Randall and R. Sundrum, Phys. Rev. Lett. 83, 4690 (1999).

[9] P. Binétruy, C. Deffayet, U. Ellwanger, and D. Langlois, Phys. Lett. B 477, 285 (2000); C. Csaki, M. Graesser, C. Kolda, and J. Terning, ibid. 462, 34 (1999); C. Csaki, M. Graesser, L. Randall, and J. Terning, Phys. Rev. D 62, 045015 (2000).

[10] J. Maldacena, Adv. Theor. Math. Phys. 2, 231 (1998); S. Gubser, I. Klebanov, and A. Polyakov, Phys. Lett. B 428, 105 (1998); E. Witten, Adv. Theor. Math. Phys. 2, 253 (1998).

[11] J. Maldacena (unpublished); E. Witten (unpublished); S. Gubser, Phys. Rev. D 63, 084017 (2001); S. Hawking, J. Maldacena, and A. Strominger, J. High Energy Phys. 05, 001 (2001); L. Anchordoqui, C. Nunez, and K. Olsen, ibid. 10, 050 (2000).

[12] N. Arkani-Hamed, S. Dimopoulos, N. Kaloper, and R. Sundrum, Phys. Lett. B 480, 193 (2000); S. Kachru, M. Schulz, and E. Silverstein, Phys. Rev. D 62, 045021 (2000); C. Schmidhuber, Nucl. Phys. B580, 140 (2000); B585, 385 (2000); C.P. Burgess, R.C. Myers, and F. Quevedo, Phys. Lett. B 495, 384 (2000); S.P. de Alwis, A.T. Flournoy, and N. Irges, J. High Energy Phys. 01, 027 (2001); S. Forste, Z. Lalak, S. Lavignac, and H. Peter Nilles, ibid. 09, 034 (2000); P. Binetruy, J.M. Cline, and C. Grojean, Phys. Lett. B 489, 403 (2000); J.E. Kim, B. Kyae, and H.M. Lee, "A Model for Selftuning the Cosmological Constant," hep-th/0011118; N. Tetra- dis, "On Brane Stabilization and the Cosmological Constant," hep-th/0012106.

[13] A. Einstein, Sitz. der Preuss. Akad. d. Wissen. (1919), translation in The Principle of Relativity (Methuen, London, 1923), reprinted by Dover.

[14] J.J. Van der Bij, H. van Dam, and Y.J. Ng, Physica A 116, 307 (1982).

[15] S. Weinberg, in Workshop on Problems in Unification and Supergravity, La Jolla Institute, 1983; F. Wilczek and A. Zee, in High Energy Physics: Proceedings of the Annual Orbis Scientiae, edited by Mintz and A. Perlmutter (Plenum, New York, 1985); W. Buchmuller and N. Dragon, Phys. Lett. B 207, 292 (1988).

[16] W.G. Unruh, Phys. Rev. D 40, 1048 (1989).

[17] M. Henneaux and C. Teitelboim, Phys. Lett. B 222, 195 (1989).

[18] A. Aurilia, H. Nicolai, and P.K. Townsend, Nucl. Phys. B176, 509 (1980).

[19] M.J. Duff and P. van Nieuwenhuizen, Phys. Lett. 94B, 179 (1980); E. Witten, in Proceedings of the 1983 Shelter Island Conference on Quantum Field Theory and the Fundamental Problems of Physics, edited by R. Jackiw, N.N. Khuri, S. Weinberg, and E. Witten (MIT, Cambridge, MA, 1983); M. Henneaux and C. Teitelboim, Phys. Lett. 143B, 415 (1984).

[20] E. Bergshoeff, M. de Roo, M.B. Green, G. Papadopoulos, and P.K. Townsend, Nucl. Phys. B470, 113 (1996).

[21] N. Alonso-Alberca, P. Meessen, and T. Ortin, Phys. Lett. B 482, 400 (2000).

[22] M. Cvetic, H. Lu, and C.N. Pope, Class. Quantum Grav. 17, 4867 (2000); E. Bergshoeff, R. Kallosh, and A. Van Proeyen, J. High Energy Phys. 10, 033 (2000); A. Falkowski, Z. Lalak, and S. Pokorski, Phys. Lett. B 491, 172 (2000); "Five Dimensional Gauged Supergravities with Universal Hypermultiplet and Warped Brane World," hep-th/0009167; M.J. Duff, J.T. Liu, and K.S. Stelle, "A Supersymmetric Type IIB RandallSundrum Realization,” hep-th/0007120; M. Cvetic, M.J. Duff, J.T. Liu, H. Lu, C.N. Pope, and K.S. Stelle, "Randall-Sundrum Brane Tension," hep-th/0011167.

[23] See, e.g., J. Polchinski, String Theory (Cambridge University Press, Cambridge, England, 1998).

[24] W. Israel, Nuovo Cimento Soc. Ital. Fis., B 44, 1 (1966).

[25] H.A. Chamblin and H.S. Reall, Nucl. Phys. B562, 133 (1999); N. Kaloper, Phys. Rev. D 60, 123506 (1999); T. Nihei, Phys. 
Lett. B 465, 81 (1999); H.B. Kim and H.D. Kim, Phys. Rev. D 61, 064003 (2000).

[26] H. Stoica, S.-H.H. Tye, and I. Wasserman, Phys. Lett. B 482, 205 (2000).

[27] J.M. Cline, C. Grojean, and G. Servant, Phys. Rev. Lett. 83, 4245 (1999); T. Shiromizu, K. Maeda, and M. Sasaki, Phys. Rev. D 62, 024012 (2000).

[28] P. Kraus, J. High Energy Phys. 12, 011 (1999); E.E. Flanagan, S.-H.H. Tye, and I. Wasserman, Phys. Rev. D 62, 044039 (2000); P. Binétruy, C. Deffayet, U. Ellwanger, and D. Langlois, Phys. Lett. B 477, 285 (2000).

[29] P. Horava and E. Witten, Nucl. Phys. B460, 506 (1996); B475, 94 (1996).

[30] J. Lykken and L. Randall, J. High Energy Phys. 06, 014 (2000).

[31] See, e.g., J. de Boer, E. Verlinde, and H. Verlinde, J. High Energy Phys. 08, 003 (2000), and references therein.

[32] S. Mukohyama, T. Shiromizu, and K. Maeda, Phys. Rev. D 62, 024028 (2000); M. Cvetic and J. Wang, ibid. 61, 124020 (2000); P. Bowcock, C. Charmousis, and R. Gregory, Class. Quantum Grav. 17, 4745 (2000).

[33] S.W. Hawking and G.F.R. Ellis, The Large Scale Structure of Space-Time (Cambridge University Press, Cambridge, England, 1973).
[34] C. Csaki, J. Erlich, and T.J. Hollowood, Phys. Rev. Lett. 84, 5932 (2000).

[35] T. Tanaka and X. Montes, Nucl. Phys. B582, 259 (2000).

[36] B. Grinstein, D.R. Nolte, and W. Skiba, Phys. Rev. D 63, 105005 (2001).

[37] J. Garriga and T. Tanaka, Phys. Rev. Lett. 84, 2778 (2000).

[38] S. Giddings, E. Katz, and L. Randall, J. High Energy Phys. 03, 023 (2000).

[39] Z. Kakushadze, Phys. Lett. B 497, 125 (2001); A. Neronov and I. Sachs, "On Metric Perturbations in Brane-World Scenarios," hep-th/00111254; N. Deruelle, T. Dolezel, and J. Katz, Phys. Rev. D 63, 083513 (2001).

[40] C. Csaki and Y. Shirman, Phys. Rev. D 61, 024008 (2000).

[41] R.M. Wald, General Relativity (University of Chicago, Chicago, 1984).

[42] P.A.M. Dirac, Proc. R. Soc. London A338, 439 (1974).

[43] C.H. Brans and R.H. Dicke, Phys. Rev. 124, 925 (1961); R.H. Dicke, ibid. 125, 2163 (1962).

[44] Ya.B. Zel'dovich, Usp. Fiz. Nauk 95, 209 (1968) [Sov. Phys. Usp. 11, 381 (1968)].

[45] M.J. Duff and J.T. Liu, Phys. Rev. Lett. 85, 2052 (2000).

[46] D.Z. Freedman, S.S. Gubser, K. Pilch, and N.P. Warner, Adv. Theor. Math. Phys. 3, 363 (1999); M. Henningson and K. Skenderis, J. High Energy Phys. 07, 023 (1998). 